\title{
On the co-existence of maximal and whiskered tori in the planetary three-body problem
}

\author{
Gabriella Pinzari ${ }^{1,}$ a) \\ Dipartimento di Matematica Tullio Levi-Civita, Università di Padova, \\ Italy
}

In this paper we discuss about the possibility of coexistence of stable and unstable quasi-periodic KAM tori in a region of phase space of the three-body problem. The argument of proof goes along KAM theory and, especially, the production of two non smoothly related systems of canonical coordinates in the same region of the phase space, the possibility of which is foreseen, for "properly-degenerate" systems, by a theorem of Nekhorossev and Miščenko and Fomenko. The two coordinate systems are alternative to the classical reduction of the nodes by Jacobi, described, e.g., in [V.I. Arnold, Small denominators and problems of stability of motion in classical and celestial mechanics, 18, 85 (1963); p. 141].

PACS numbers: 02. Mathematical methods in physics

a) gabriella.pinzari@math.unipd.it 


\section{INTRODUCTION}

At the end of the XIX century, motivated by the study of a three-body problem of celestial mechanics, H. Poincaré conjectured that a non-integrable system possesses, very close one to the other, stable and unstable trajectories, Ref. ${ }^{36}$ (Vol III, Chapter 33, n. 397, p. 389). Numerical evidence of such an occurrence has been provided, since the 50s, as soon as computers could be used to simulate solutions of differential equations, by breakthrough papers by Fermi-Pasta-Ulam, Hénon-Heiles, Izrailev-Chirikov, .... The phenomenon was soon understood to be relevant for physics, since such papers revealed its appearance even in regular (e.g. analytic) systems. At this respect, the recent development of AubryMather theory allowed to obtain, under strong assumptions ${ }^{41}$, still failing to be applied to celestial mechanics, the rigorous proof of existence of motions with any prescribed frequency. In this paper, we address the question in the case of the celestial three-body problem; precisely, its planetary version. This is the 4 degrees of freedom problem of three point masses interacting through gravity, where one of the masses (the "star") is much larger than the two others (the "planets"). We move in the framework of Kolmogorov-Arnold-Moser (KAM) theory; Refs. ${ }^{2,21,29}$ and Note ${ }^{42}$. KAM theory has been successfully applied to problem of celestial mechanics it since the 60s. Under the point of view of KAM theory, the question might be rephrased as wether one can prove coexistence, in a region of phase space, of quasi--

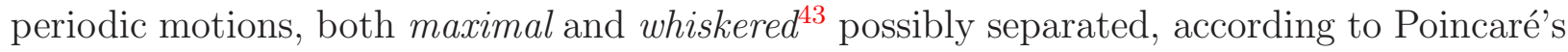
picture and Aubry-Mather theory, by chaotic regions. This is precisely the question to which this paper is addressed: we determine a physical situation where such co-existence is expected to occur. We consider the following situation, which we shall refer to as outer retrograde configuration (ORC): two planets describe almost co-planar orbits. The outer planet has a retrograde motion ${ }^{44}$.

Before describing our result, let us make a digression on the specific features planetary systems and previous literature. It is known that the two-body problem, i.e., the problem of the motions of two point masses interacting via a law proportional to their inverse squared distance, has, for an open set of initial conditions, periodic motions rather than, more generally, quasi-periodic. For this system, periodicity consists in the fact that the bounded motions evolve (according to Kepler's laws) on ellipses, and are governed by just one frequency $\nu$ proportional to $a^{-3 / 2}$ where $a$ is the semi-major axis of the ellipse. This 
pretty remarkable fact unavoidably reflects - as already underlined by V. I. Arnold in his 1963's paper Ref. ${ }^{3}$ - on the study of the dynamics of the so-called planetary problem, i.e., the problem of $(1+N)$ point masses, one of which ("sun") is of "order one", while the remaining $N$ ("planets") are of much smaller size, interacting through gravity. Indeed, when the reciprocal attraction among the planets, which is of much smaller order compared to the attraction between any planet and the sun, is set to zero, the planetary problem reduces to $N$ uncoupled two-body problems ("unperturbed problem"). He considered the case of $N$ planets in prograde configuration; i.e., revolving in the same verse, even though the question of the sense of rotation, at his time, was definitely of secondary importance, compared to the difficulties that had to be overcome and that we are going to recall.

The lack of frequencies(a translationally invariant system with $1+N$ bodies possesses $3 N$ degrees of freedom. In the case of the planetary problem, it exhibits, as mentioned, only $N<3 N$ frequencies) in the unperturbed problem was named by Arnold proper degeneracy. It represented a serious difficulty, if one wanted (as he was aiming to do) to apply Kolmogorov's theorem, Ref. ${ }^{21}$ to the planetary problem.

At a technical level, the appearance of the proper degeneracy consists, we might say, of a "loss of frequencies", caused by the "too many" (or, better Poisson non commuting ${ }^{45}$, see below) first integrals of motion. For such abundance, this kind of systems is often called super-integrable. Despite of the fact that the solutions of the two-body problem are known since Newton's times, a general, theoretical setting clearly explaining the phenomenon has been given only recently, thanks to the works by Nekhorossev and Miščenko and Fomenko, Refs. ${ }^{27,30}$ (hereafter, NMF). The three authors proved a generalization of the best known Liouville-Arnold theorem, Ref. ${ }^{1}$ which clearly relates the loss of frequencies to the existence of Poisson non commuting independent integrals. They proved that, to an integrable Hamiltonian system with $n$ degrees of freedom which, in addition to $n$ independent and commuting first integrals, affords additional, independent, from the first $n, n_{1} \leq n$ integrals which do not commute with all the integrals of the first family, one can associate canonical coordinates including only $n_{0}:=n-n_{1}$ action-angle pairs $(\mathrm{I}, \varphi)=\left(\mathrm{I}_{1}, \cdots, \mathrm{I}_{n_{0}}, \varphi_{1}, \cdots, \varphi_{n_{0}}\right)$ (analogous to the ones of Arnold-Liouville case), and, in addition, certain other couples $z=(p, q)=\left(p_{1}, \cdots, p_{n_{1}}, q_{1}, \cdots, q_{n_{1}}\right)$, usually referred to as degenerate coordinates. The degenerate coordinates are not uniquely defined, and this is precisely the aspect that, in this paper, we shall exploit. 
Indeed, a dynamical system that is close to a super-integrable system may be written as

$$
\mathrm{H}(\mathrm{I}, \varphi, p, q)=\mathrm{h}(\mathrm{I})+\mu f(\mathrm{I}, \varphi, p, q)
$$

where $(\mathrm{I}, \varphi, p, q)$ is one of the various (as foreseen by NMF Theorem) sets of canonical coordinates associated with the unperturbed super-integrable term h. Now, while, given the I's, h is uniquely determined, the form of $f$, instead, strongly depends on the choice of coordinates. On the other hand, it is known since Arnold's paper Ref. ${ }^{3}$ that, for system of the form (1), $f$ may have a strategic importance.

As an outstanding example, let us recall just the case considered by Arnold in Ref. ${ }^{3}$. He wanted to prove (via an application of Kolmogorov's theorem) the existence of plenty of quasi-periodic, maximal tori, forming a positive measure set in phase space. He announced the result (known as "Arnold Theorem") at the 1962 ICM. Clearly, such result was going in the direction of the proof of stability of the Solar System, and for this Kolmogorov and Arnold were awarded, in 1965, of the Lenin prize. However, in order to obtain such result he was aware that he had to overcome the problem of the lack of frequencies in the unperturbed part (indeed succeeding in this), but this was not the only one. As for the choice of coordinates, Arnold considered, in the case of the planar problem, Poincaré coordinates, as described in Ref. $^{3}$ (Chapter III, $§ 2$, n.4). In term of such coordinates, the Hamiltonian of the planetary problem takes the form in (1), with $n_{0}$ equal to the number of planets $N, n_{1}=N$ (so that the total number of degrees of freedom in $\mathbf{R}^{2}$ is $\left.2 N\right),(\mathrm{I}, \varphi):=(\Lambda, \lambda) \subset \mathbf{R}^{N} \times \mathbf{T}^{N}$ (where $\mathbf{T}:=\mathbf{R} /(2 \pi \mathbf{Z})$ ) suitable action-angle couples related to the semi-major axis and the area spanned by the ellipse, $z=(p, q):=(\eta, \xi) \subset \mathbf{R}^{N} \times \mathbf{R}^{N}$ suitable degenerate coordinates related to the orientation of such ellipses, $\mathrm{h}=\mathrm{h}_{\mathrm{k}}$ the Keplerian Hamiltonian; $\mu$ a small a-dimensional parameter measuring the maximum planet/star mass ratio and, finally, $f(\mathrm{I}, \varphi, p, q)=f_{\mathrm{Poin}}(\Lambda, \lambda, \eta, \xi)$ a perturbing function related to the small mutual interactions among planets. Arnold observed that the average value $\overline{f_{\text {Poin }}}(\Lambda, \eta, \xi)$ with respect to the $\lambda$ 's of the perturbing function $f_{\mathrm{Poin}}(\Lambda, \lambda, \eta, \xi)$ by symmetry reasons, has an elliptic equilibrium point for $(\eta, \xi)=0$ (corresponding to circular motions of the planets around their sun), for all $\Lambda$. So he managed to construct, for degenerate systems of the form (1) with $\bar{f}(I, z)$ having an elliptic equilibrium in $z=0$ for all $\mathrm{I}$, a careful version of Kolmogorov Theorem, Ref. ${ }^{3}$ (Fundamental Theorem) based on a generalized non-degeneracy condition ("full torsion"), inspired to Kolmogorov, according to which one should check, besides of 
the non-singularity of the Hessian matrix $\partial_{\mathrm{I}_{i} \mathrm{I}_{j}}^{2} \mathrm{~h}(I)$ in $(1)$ also the one of the matrix of the coefficients of the second-order term of the Birkhoff normal form associated to the elliptic equilibrium (see Ref. ${ }^{18}$ ). Arnold successfully applied his Fundamental Theorem to the case of the planar problem with $N=2$ planets. However, while the extension to the planar problem with a generic number of planets revealed to be straightforward, Ref. ${ }^{34}$ (see Ref. ${ }^{12}$ for a previous result with a different strategy), the treatment of the problem in space contained strong extra-difficulties. Indeed, switching from planar to spatial Poincaré coordinates, the averaged perturbing function $\overline{f_{\text {Poin }}}$ still exhibits an elliptic equilibrium in correspondence circular and co-planar motions, but such equilibrium is degenerate, in the sense that the eigenvalues of the quadratic part of $\overline{f_{\text {Poin }}}$ verify, identically, two linear combinations with integer coefficients (known in the field as secular resonances). A fact strongly preventing, in principle, the construction of the Birkhoff normal form and hence the possibility of checking the full torsion condition. But this is not all: a formal evaluation of the torsion, attempted asymptotically by M. Herman, Ref. ${ }^{17}$, seemed to suggest, in absence of proper reductions of the rotation invariance, an identically vanishing determinant (implying the impossibility of applying the Fundamental Theorem to the general problem), a fact next rigorously proved, by L. Chierchia and the author, in Ref. ${ }^{8}$.

It may be argued that Arnold felt that a difficulty of this kind might appear, since, without explaining his motivations, in Ref. ${ }^{3}$ (p. 141-42), he suggested to "change coordinates", without going further. Completion of the proof of his theorem revealed it to be more difficult than expected, and the story reached a conclusion only fifty years later, thanks to contributions by J. Laskar, P. Robutel, M. Herman, J.Féjoz, L. Chierchia and the author, Refs. 9,12,22,34,39. Comprehensive reviews appeared in Refs. ${ }^{10,13}$, to which papers we refer the interested reader. For the purposes of this paper we only mention that the solution Arnold had in mind, based on changing coordinates was considered, formally, in a particular case, by Malige, Robutel and Laskar, Ref. ${ }^{25}$, and next completely achieved by the author, Ref. ${ }^{34}$, published in Refs. ${ }^{9,34}$. The long proof of Arnold Theorem should give, we hope, an idea that, from a practical point of view, producing "good" canonical coordinates, which should: (i) leave the unperturbed part unvaried; (ii) overcome the degeneracies caused by $\mathrm{SO}(3)$ invariance and, eventually, (iii) preserve symmetries, parities, equilibria ... from which to depart in order to apply a perturbative scheme (e.g., in the case of Arnold Theorem, the Fundamental Theorem developed around the elliptic equilibrium), is other than "easy" or "straightforward". 
In this paper, we look at the three-body problem in the ORC configuration by means of (basically) two sets of canonical coordinates. The former of such two systems of coordinates is a modification of the so-called "regular, planetary and symplectic" (rps) coordinates, proposed in Refs. ${ }^{9,34}$. The latter, called "perihelia reduction" (p) has been proposed in Ref..$^{33}$. Both such systems of coordinates describe regularly co-planar motions, which evolve on suitable invariant manifolds of each phase space. Each of such invariant manifolds turns to be an equilibrium for suitable truncated and averaged systems (where the average is performed with respect to fast angle coordinates in each of such sets), parametrized by the value of certain other action coordinates, which play the rôle of quasi-integrals of motion.

We provide the complete proof of the existence of a positive measure set with a maximal number of frequencies for the full system, both in the case of planar and spatial problem. More precisely, we prove the following result (a more precise formulation will be given in course of the paper; see Theorem III.2).

Theorem There exists an eight-dimensional open region of phase space, contained in the holomorphy domain of the Hamiltonian, almost completely filled with a positive measure set of quasi-periodic motions with maximal number of incommensurate frequencies. The motions on such tori are in ORC.

The proof of the Theorem adapts the techniques of Refs. ${ }^{9,34}$, which, as recalled above, dealt with the prograde case. Although the strategy is the same, nevertheless, certain structural differences between the two settings do exist, which we point out. The most remarkable one is related to the effect of the rotation invariance in the two cases, in relation with the elliptic nature of the co-planar, co-circular equilibrium. While, in the case treated in Refs. ${ }^{9,34}$, such ellipticity is a mere consequence of its invariance by reflections and rotations (a fact already known to M. Herman, Ref. ${ }^{17}$ ), in the retrograde case, it is not so, but needs to be checked specifically.

We conclude this introduction with recalling related literature.

The existence of a positive measure set of Lagrangian tori with maximal number (see the next section) of frequencies for the general planetary problem, in the regime of well spaced orbits, small eccentricities and small inclinations, has been established in the papers Refs. ${ }^{3,9,12,22,33-35}$. We refer to such technical papers for details, to Refs. ${ }^{10,13}$ for reviews. However, the cases treated in the literature above, even though containing all the necessary 
information, are not perfectly suited to the proof of the the Theorem.

The papers in Refs. ${ }^{3,9,12,22,34,39}$ deal with maximal quasi-periodic tori in the case when the planets revolve all in the same verse, and eccentricities and inclinations are small. The invariant set (so-called "Kolmogorov-set") for the (rescaled) Hamiltonian in (2) is proved to fill almost completely the region in phase space with small eccentricities and inclinations, up to a residual set with measure going to zero with the parameter $\mu$ in (2) and with the maximum $\varepsilon$ of eccentricities and inclinations. A (maybe optimal) estimate about the strength at which such measure goes to zero is provided in Refs. ${ }^{9,34}$.

In Refs. ${ }^{33,35}$ maximal quasi-periodic tori have been constructed out of the small eccentricities and inclination constraint. In such papers, the measure of the Kolmogorov-set has been found to increase while the masses decrease and the mutual semi-major axes ratios increase, independently of the values of eccentricities and inclinations. A suitable constraint on the semi-axes ratios is however imposed. Finally, the sense of revolution of the planets is the same for all of them.

A study of quasi-periodic motions (including retrograde ones) bifurcating from relative equilibria appeared in Refs. ${ }^{31,32}$. However, the measure estimates obtained in those papers, based on a bit different framework (Withney regularity and no use of Birkhoff normal form; see, e.g., Ref. ${ }^{32}$ (Theorem 5.1)), are not suited to the purposes of the paper, where the point of view is closer to Ref. ${ }^{3}$.

\section{SET UP}

The three-body problem is the dynamical system formed by three point masses in $\mathbf{R}^{3}$ interacting through gravity only. The system has thus nine degrees of freedom, meaning with this that its evolution is described by a system of differential equations having order eighteen. However, it is also well known that the system possesses several constant motions, and, even though the number of such constant is not sufficient (as Poincaré showed, Ref. ${ }^{36}$ ) to guarantee integrability by quadratures, nevertheless, it allows to reduce the order of the equations from eighteen to eight. This complete reduction was firstly considered by Jacobi and refined by R. Radau, Refs. ${ }^{19,38}$ (see the next section). The first step to achieve it consists of getting free of the translation invariance, caused by the conservation of the total linear momentum (the velocity of the center of mass of the system). In literature one finds essentially two 
ways to do it, usually referred to as "Jacobi" or "heliocentric" coordinates. They both can be described as linear changes of coordinates, if the Hamiltonian of the system is initially written in impulse-position coordinates. We refer to Refs. ${ }^{3,39}$ for a complete description of them. According to the heliocentric reduction, in a system where the masses are denoted as $m_{0}, \mu m_{1}, \mu m_{2}$, where $\mu$ is a prefixed pure number (the case $\mu \ll 1$ and $m_{i}$ 's of the same strength being usually referred as "planetary" problem), the motions are described the Hamilton equations of the six degrees of freedom Hamiltonian

$$
\mathrm{H}(y, x)=\frac{\left|y^{(1)}\right|^{2}}{2 \mathrm{~m}_{1}}-\frac{\mathrm{m}_{1} \mathrm{M}_{1}}{\left|x^{(1)}\right|}+\frac{\left|y^{(2)}\right|^{2}}{2 \mathrm{~m}_{2}}-\frac{\mathrm{m}_{2} \mathrm{M}_{2}}{\left|x^{(2)}\right|}+\mu\left(-\frac{m_{1} m_{2}}{\left|x^{(1)}-x^{(2)}\right|}+\frac{y^{(1)} \cdot y^{(2)}}{m_{0}}\right)
$$

where

$$
\mathrm{m}_{i}:=\frac{m_{0} m_{i}}{m_{0}+\mu m_{i}}=m_{i}+\mathrm{O}(\mu) \quad \mathrm{M}_{i}:=m_{0}+\mu m_{i}=m_{0}+\mathrm{O}(\mu)
$$

are the "reduced masses"; $y^{(i)} \in \mathbf{R}^{3}, x^{(i)} \in \mathbf{R}^{3}$, and the collision set

$$
\Delta:=\left\{x^{(1)}=0, \text { or } x^{(2)}=0, \text { or } x^{(1)}=x^{(2)}\right\}
$$

is to be excluded. Incidentally, the two terms in the perturbing function are sometimes referred to as direct (or Newtonian), indirect part, respectively.

After the linear momentum reduction, the next issue is to get rid of rotation invariance of the Hamiltonian (2), caused by the conservation of the three components $\mathrm{C}_{1}, \mathrm{C}_{2}$ and $\mathrm{C}_{3}$ of total angular momentum of the system:

$$
\mathrm{C}=\mathrm{C}^{(1)}+\mathrm{C}^{(2)} \quad \text { with } \quad \mathrm{C}^{(i)}:=x^{(i)} \times y^{(i)} .
$$

This further step is more subtle than the previous one for two reasons. The first obvious reason is that, differently from the linear momentum reduction, it cannot be obtained via a linear transformation. But the main reason is that, in the case of the problem in $\mathbf{R}^{3}$, the $\mathrm{C}_{i}$ 's do not Poisson-commute, one cannot think to a elimination "by quadratures" (i.e., one cannot think, roughly speaking, to use them as generalized momenta, mimicking the linear momentum reduction procedure). The only widely known method in the field (recalled in the next section) is due to C. G. Jacobi, R. Radau and, after the work of A. Deprit (see the next section), it is available for any number of particles. Another reduction, called "reduction of perihelia", has been recently proposed in Ref. ${ }^{33}$ and, as well as the previous one, is available for any number of bodies. It will be recalled in Section IV in the particular case of three bodies. For the results of the paper, both the mentioned reductions will be used. A new unified proof of their canonical character is presented in Section V. 


\section{A. The Jacobi-Radau-Deprit coordinates}

In the XIX Century C.G.Jacobi, Ref. ${ }^{19}$, found a tricky procedure, that is usually referred to as "Jacobi's reduction of the nodes" that allowed him to write the differential equations of the spatial three-body problem as a system of order eight. His speculations were refined by R. Radau, Ref. ${ }^{38}$, who wrote such equations as a system of eight equations of order one, corresponding to the Hamilton equations of a four degrees of freedom Hamiltonian. Even though the original Jacobi-Radau's work was suited for a general two-particles system enjoying rotation invariance, it is customary (compare, e.g., Ref. ${ }^{3}$ ) to refer with the same name a slightly modified procedure such in a way that the -integrable - (translationally reduced) two-body terms

$$
\frac{\left|y^{(j)}\right|^{2}}{2 \mathrm{~m}_{j}}-\frac{\mathrm{m}_{j} \mathrm{M}_{j}}{\left|x^{(j)}\right|}
$$

appearing in the Hamiltonian (2), are put in action-angle form, in the sense of LiouvilleArnold Theorem, Ref. ${ }^{1}$. As known, the Liouville-Arnold form for (5) is one-dimensional

$$
\mathrm{h}_{\mathrm{k}}^{(j)}\left(\Lambda_{j}\right):=-\frac{\mathrm{m}_{j}^{3} \mathrm{M}_{j}^{2}}{2 \Lambda_{j}^{2}},
$$

with the action $\Lambda_{j}$ 's being related to the semi-major axis $a_{i}$ of the Keplerian ellipse via

$$
\Lambda_{j}=\mathrm{m}_{j} \sqrt{\mathrm{M}_{j} a_{j}} .
$$

Jacobi's trick consisted in fixing in advance a rotating reference frame having its third axis in the (constant) direction of the total angular momentum $\mathrm{C}$ and its first axis in the (moving) direction of the so-called "nodes lines". Astronomers call so the straight line determined by the intersection (provided it is well defined) of the instantaneous planes of of the orbits of the two planets; i.e., the planes $\Pi_{j}(t)=\left(y^{(j)}(t), x^{(j)}(t)\right), j=1$, 2. Jacobi and Radau proved that, even though the reference frame moves, nevertheless, the system of eight coordinates given by the "planar Delaunay elements" (see Ref. ${ }^{15}$ )

$$
\mathrm{j}=\left(\Lambda_{1}, \Lambda_{2}, \mathrm{G}_{2}, \mathrm{G}_{2}, \ell_{1}, \ell_{2}, \gamma_{1}, \gamma_{2}\right)
$$

on the moving planes $\Pi_{1}(t), \Pi_{2}(t)$ induces an injection

$$
\phi_{\mathrm{j}}: \quad \mathrm{j}=\left(\Lambda_{1}, \Lambda_{2}, \mathrm{G}_{2}, \mathrm{G}_{2}, \ell_{1}, \ell_{2}, \gamma_{1}, \gamma_{2}\right) \in \mathbf{R}^{4} \times \mathbf{T}^{4} \rightarrow \mathcal{C}=\left(y^{(1)}, y^{(2)}, x^{(1)}, x^{(2)}\right) \in \mathbf{R}^{12}
$$

such that the motion of the system are the solutions of the Hamilton Equation of $\mathrm{H}_{\mathrm{j}}:=\mathrm{H} \circ \phi_{\mathrm{j}}$, which, moreover, turns to depend parametrically only of the length $\mathrm{G}=|\mathrm{C}|$ (namely, it does 
not depend on the direction of $\mathrm{C}$; a fact vaguely attributed, at that time, to the rotation invariance of the system, but next fully understood thanks to the work of A. Deprit, much years later; see below). The reduction by Jacobi and Radau was well known to V.I.Arnold, who mentioned it in $^{3}$ (II, $\S 5$, n.4, p.141) as a unavoidable tool (due to certain degeneracies that appeared if such tool was not used) in order to prove the stability of the planetary, spatial three-body problem in the perturbative setting. The proof of stability that he had discovered consisted of checking the non-singularity of a certain matrix ("torsion"; see Section III B) related to a certain averaged perturbing function. However, having treated with much detail the case of the planar problem (which had required a considerable amount of computation to him), in order to simplify the analysis, instead of computing the torsion of the spatial problem directly, he preferred to try to reduce the spatial problem to a perturbed planar one, so as to use the computations he had already done. This led him in error (roughly, because Jacobi's reduction is singular for co-planar motions). The computation of the torsion for the spatial problem, using Jacobi's reduction of the nodes, was next completed by Laskar and Robutel; Refs. ${ }^{22,39}$.

How to obtain a generalization of Jacobi-Radau coordinates to the case of more than three bodies has been a one century long problem of mechanics, that Arnold invoked as an obstruction to the extension of his outline of proof to the general planetary problem in the space, Ref. ${ }^{3}$ (Ch. III, $§ 5$, n.5, p. 141). A mild progress was offered only in 1982 by F. Boigey who, during her PhD, obtained a Jacobi-like reduction for the problem of four bodies, Ref. ${ }^{5}$. One year after, in 1983, and twenty years after Arnold's work, A. Deprit, positively impressed (as he declared in the introduction) by Boigey's work, discovered a set of canonical coordinates that (in a sense; see below) reduces to Jacobi-Radau's for $N=2$ and to Boigey's for $N=3$. For some strange reason, Deprit's work was overlooked ${ }^{46}$ for further twenty years and Deprit himself seemed to be not much confident about the utility of his coordinates ${ }^{47}$. During such time, the complete proof of Arnold's Theorem had been obtained by HermanFéjoz, Refs. ${ }^{12,17}$, with a different strategy (the problem of degeneracies in the Hamiltonian was overcome with an abstract argument of Lagrangian mechanics, without using explicit coordinates). Deprit's work was rediscovered by the author during her PhD, precisely in the framework of obtaining a proof of Arnold's theorem accordingly to the original strategy. The production, in ${ }^{34}$ (published in Ref. ${ }^{9}$ ) of a new set "regularizing" coordinates (see also the next section) much similar to Poincaré's coordinates, but better suited to bypass the 
problem of degeneracies, was the final key to reach the objective.

As in the case of Jacobi's reduction, it is customary to call "Deprit's coordinates" a modified version (in fact, the form they were rediscovered in Ref. ${ }^{34}$ ) of the original set discussed in Ref. ${ }^{11}$, such in a way to satisfy (6). We recall such modified version, in the case of a system of two particles.

We fix a domain $\mathcal{D}_{\text {jrd }} \subset \mathbf{R}^{12}$ in phase space as follows. Let $\left(k^{(1)}, k^{(2)}, k^{(3)}\right)$ be a prefixed orthonormal frame in $\mathbf{R}^{3}$. For the Cartesian coordinates $\mathcal{C}_{\text {art }}=\left(y^{(1)}, y^{(2)}, x^{(1)}, x^{(2)}\right) \in \mathcal{D}_{\text {jrd }}$, we assume that the orbits $t \rightarrow\left(x^{(j)}(t), y^{(j)}(t)\right)$ generated by the Hamiltonians (5) with initial datum $\left(x^{(j)}, y^{(j)}\right)$ are ellipses with non-vanishing eccentricity, belonging to different planes, never coinciding with the $(1,2)$ plane. Then we denote as $\mathrm{P}^{(j)}$ the unit vectors pointing in the directions of the perihelia; as $a_{j}$ the semi-major axes; as $\ell_{j}$ the "mean anomaly" of $x^{(j)}$ (which, we recall, is defined as area of the elliptic sector from $\mathrm{P}^{(j)}$ to $x^{(j)}$ "normalized at $\left.2 \pi^{\prime \prime}\right)$; as $\mathrm{C}^{(j)}=x^{(j)} \times y^{(j)}, j=1,2$, the angular momenta of the two planets and $\mathrm{C}:=\mathrm{C}^{(1)}+\mathrm{C}^{(2)}$ the total angular momentum integral. By assumption, the "nodes"

$$
\nu_{1}:=k^{(3)} \times \mathrm{C}, \quad \nu:=\mathrm{C} \times \mathrm{C}^{(1)}=\mathrm{C}^{(2)} \times \mathrm{C}^{(1)}
$$

do not vanish. For three vectors $u, v, w$ with $u, v \perp w$, we denote as $\alpha_{w}(u, v)$ the angle formed by $u$ to $v$ relatively to the positive (counterclockwise) orientation established by $w$. The jrd $:=\phi_{\mathrm{jrd}}^{-1}\left(\mathcal{C}_{\text {art }}\right)$ coordinates are defined via the following formulae:

$$
\left\{\begin{array} { l } 
{ Z : = \mathrm { C } \cdot k ^ { ( 3 ) } } \\
{ \mathrm { G } : = \| \mathrm { C } \| } \\
{ \mathrm { G } _ { 1 } : = \| \mathrm { C } ^ { ( 1 ) } \| } \\
{ \mathrm { G } _ { 2 } : = \| \mathrm { C } ^ { ( 2 ) } \| } \\
{ \Lambda _ { j } : = \mathrm { M } _ { j } \sqrt { \mathrm { m } _ { j } a _ { j } } }
\end{array} \quad \left\{\begin{array}{l}
\zeta:=\alpha_{k^{(3)}}\left(k^{(1)}, \nu_{1}\right) \\
\gamma:=\alpha_{\mathrm{C}}\left(\nu_{1}, \nu\right) \\
\gamma_{1}:=\alpha_{\mathrm{C}^{(1)}}\left(\nu, \mathrm{P}^{(1)}\right) \\
\gamma_{2}:=\alpha_{\mathrm{C}^{(2)}}\left(\nu, \mathrm{P}^{(2)}\right) \\
\ell_{j}:=\text { mean anomaly of } x^{(j)}
\end{array}\right.\right.
$$

Note that such definitions, together with assumptions of non-vanishing of the nodes (10), imply that

$$
\min \left\{\mathrm{G}_{1}, \mathrm{G}_{2}, \mathrm{G}\right\}>0, \quad-\mathrm{G}<\mathrm{Z}<\mathrm{G}, \quad\left|\mathrm{G}_{1}-\mathrm{G}_{2}\right|<\mathrm{G}<\mathrm{G}_{1}+\mathrm{G}_{2} .
$$

The coordinates (11) will be denoted in this paper as

$$
\text { jrd }:=\left(\Lambda_{1}, \Lambda_{2}, \mathrm{G}_{1}, \mathrm{G}_{2}, \mathrm{G}, \mathrm{Z}, \ell_{1}, \ell_{2}, \gamma_{1}, \gamma_{2}, \gamma, \zeta\right)
$$


by the names of their discoverers. Observe, for comparison, that, while (9) is a canonical injection, the (13)'s come out from a canonical change of coordinates

$$
\phi_{\mathrm{jrd}}: \quad \mathrm{jrd}=\left(\Lambda_{1}, \Lambda_{2}, \mathrm{G}_{1}, \mathrm{G}_{2}, \mathrm{G}, \mathrm{Z}, \ell_{1}, \ell_{2}, \gamma_{1}, \gamma_{2}, \gamma, \zeta\right) \rightarrow \mathcal{C}_{\mathrm{jrd}}=\left(y_{\mathrm{jrd}}^{(1)}, y_{\mathrm{jrd}}^{(2)}, x_{\mathrm{jrd}}^{(1)}, x_{\mathrm{jrd}}^{(2)}\right)
$$

i.e., which preserves the standard 2-form

$$
\sum_{j=1}^{2}\left(d \Lambda_{j} \wedge d \ell_{j}+d \mathrm{G}_{j} \wedge d \gamma_{j}\right)+d \mathrm{G} \wedge d \gamma+d \mathrm{Z} \wedge d \zeta=\sum_{j=1}^{2} \sum_{i=1}^{3} d y_{\mathrm{jrd}, i}^{(j)} \wedge d x_{\mathrm{jrd}, i}^{(j)} .
$$

From the practical point of view, there is no difference with the two, because indeed the coordinates Z, $\zeta$ and $\gamma$, which do not appear in (9), are cyclic to the Hamiltonian

$$
\mathrm{H}_{\mathrm{jrd}}:=\mathrm{H} \circ \phi_{\mathrm{jrd}}=\mathrm{h}_{\mathrm{k}}+\mu f_{\mathrm{jrd}}
$$

(where $\mathrm{H}$ is as in (2)) which in fact coincides with $\mathrm{H}_{\mathrm{j}}:=\mathrm{H} \circ \phi_{\mathrm{j}}$. Namely, in both cases, one obtains a a 10-dimensional Hamiltonian that depends on the eight coordinates in (8) and $\mathrm{G}$ but does not depend on the Deprits angle $\gamma$ conjugated to $\mathrm{G}$ (so that one can regard $\mathrm{G}$ as a parameter and regard the system as having four degrees of freedom). On the other side (and we consider this the major advantage of the (11)'s), completing the (8)'s with the quadruplet $(\mathrm{Z}, \mathrm{G}, \zeta, \gamma)$ is just what is needed to obtain a generalization to any $N \geq 2$ by induction, as shown in Ref. ${ }^{34}$ (the original proof by Deprit in Ref. ${ }^{11}$ is not by induction). The analytical form of the map (14) has beed provided in Refs. ${ }^{9,34}$, in a different framework compared to the original one proposed by Deprit (who, in Ref. ${ }^{11}$ proposed to use "quaternions"). See also Appendix A 1 a.

\section{FULL DIMENSIONAL QUASI-PERIODIC MOTIONS IN THE RETROGRADE PROBLEM}

\section{A. Analytical tools}

In this section we provide the analytical tools in order to prove the existence of full dimensional quasi-periodic motions in the retrograde problem. The proof goes through a change of coordinates that transforms the Hamiltonian (2) to a new Hamiltonian with the average with respect to the angles conjugated to the $\Lambda_{i}^{\prime} s$ being Birkhoff normal form. We

remark, at this respect that, instead of constructing directly a system of canonical coordinates that do the job and, at the same time, reduce the number of degrees of freedom to four 
(accordingly to the previous section), with the purpose of limiting the computations at a minimum, we mimic the procedure followed in Ref. ${ }^{9}$ (Sections 4-6), where the construction of the Birkhoff normal form for the fully reduced system was obtained via the passage to an intermediate system ("partial reduction") where the number of effective degrees of freedom is one over the minimum. In particular, we shall establish a correspondence between the formulae of the "prograde" case, treated in Refs. ${ }^{9,34}$, and the ones of the retrograde one, considered here. As well as in the previous literature, the coordinates that we propose have nice "regularizing properties", described in the next section.

\section{Regularizing coordinates}

The map (14), and hence the Hamiltonian (16), are not well defined when some of the following equalities is verified

$$
\begin{aligned}
& \mathrm{G}_{1}=\Lambda_{1} \quad \text { or } \quad \mathrm{G}_{2}=\Lambda_{2} \quad \text { or } \quad \mathrm{G}=\mathrm{G}_{1}+\mathrm{G}_{2} \text { or } \mathrm{G}=\mathrm{G}_{1}-\mathrm{G}_{2} \text { or } \mathrm{G}=\mathrm{G}_{2}-\mathrm{G}_{1} \\
& \text { or } \mathrm{Z}=\mathrm{G} \quad \text { or } \mathrm{Z}=-\mathrm{G} \quad \text { or } \quad \mathrm{G}_{i}=0 \quad \text { or } \mathrm{G}=0
\end{aligned}
$$

Some advantage is taken in using regularized coordinates, at expenses of introducing an extra-integral, as now we describe. The coordinates doing the job in the case of the prograde

problem have been found in Ref. ${ }^{34}$ and have been called, in Ref. ${ }^{9}$, "regular, planetary and symplectic" (rps). To avoid confusions with the coordinates that we shall introduce in a while, the rps coordinates in the case of the three-body problem will be denoted, in the paper, with the symbols

$$
\operatorname{rps}:=(\Lambda, \widetilde{\lambda}, \widetilde{\eta}, \widetilde{\xi}, \widetilde{p}, \widetilde{q}, \widetilde{P}, \widetilde{Q})=\left(\Lambda_{1}, \Lambda_{2}, \widetilde{\lambda}_{1}, \widetilde{\lambda}_{2}, \widetilde{\eta}_{1}, \widetilde{\eta}_{2}, \widetilde{\xi}_{1}, \widetilde{\xi}, \widetilde{p}, \widetilde{q}, \widetilde{P}, \widetilde{Q}\right)
$$

(they correspond to the coordinates for $n=2$ in Ref. ${ }^{9}$, and thereby named $L_{1}, L_{2}, \lambda_{1}, \lambda_{2}, \eta_{1}$, $\eta_{2}, \xi_{1}, \xi_{2}, p_{1}, q_{1}, p_{2}, q_{2}$, respectively). For later convenience, we introduce the complexified version of the (18)'s, defined as

$$
\operatorname{rps}^{\mathbf{C}}:=\left(\Lambda, \tilde{\lambda}, \widetilde{t}, \widetilde{t}^{*}, \widetilde{T}, \widetilde{T}^{*}\right)=\left(\Lambda_{1}, \Lambda_{2}, \widetilde{\lambda}_{1}, \widetilde{\lambda}_{2}, \widetilde{\tau}_{1}, \widetilde{\tau}_{2}, \widetilde{\tau}_{3}, \widetilde{t}_{1}^{*}, \widetilde{t}_{2}^{*}, \widetilde{t}_{3}^{*}, \widetilde{T}, \widetilde{T}^{*}\right)
$$

where

$$
\begin{array}{cccc}
\widetilde{t}_{1}:=\frac{\widetilde{\eta}_{1}-\mathrm{i} \widetilde{\xi}_{1}}{\sqrt{2}} & \widetilde{t}_{2}:=\frac{\widetilde{\eta}_{2}-\mathrm{i} \widetilde{\xi}_{2}}{\sqrt{2}} \quad \widetilde{t}_{3}:=\frac{\widetilde{p}-\mathrm{i} \widetilde{q}}{\sqrt{2}} \quad \widetilde{T}:=\frac{\widetilde{P}-\mathrm{i} \widetilde{Q}}{\sqrt{2}} \\
\widetilde{t}_{1}^{*}:=\frac{\widetilde{\eta}_{1}+\mathrm{i} \widetilde{\xi}_{1}}{\sqrt{2} \mathrm{i}} \quad \widetilde{t}_{2}^{*}:=\frac{\widetilde{\eta}_{2}+\mathrm{i} \widetilde{\xi}_{2}}{\sqrt{2} \mathrm{i}} \quad \widetilde{t}_{3}^{*}:=\frac{\widetilde{p}+\mathrm{i} \widetilde{q}}{\sqrt{2} \mathrm{i}} \quad \widetilde{T}^{*}:=\frac{\widetilde{P}+\mathrm{i} \widetilde{Q}}{\sqrt{2} \mathrm{i}}
\end{array}
$$


and we denote as

$$
\phi_{\mathrm{rps}}: \quad \operatorname{rps}=(\Lambda, \widetilde{\lambda}, \widetilde{\xi}, \widetilde{\eta}, \widetilde{p}, \widetilde{q}, \widetilde{P}, \widetilde{Q}) \rightarrow\left(y_{\mathrm{rps}}^{(1)}, y_{\mathrm{rps}}^{(2)}, x_{\mathrm{rps}}^{(1)}, x_{\mathrm{rps}}^{(2)}\right)
$$

respectively

$$
\phi_{\mathrm{rps}}^{\mathbf{C}}: \quad \operatorname{rps}^{\mathbf{C}}=\left(\Lambda, \tilde{\lambda}, \tilde{t}, \tilde{t}^{*}, \widetilde{T}, \widetilde{T}^{*}\right) \rightarrow\left(y_{\mathrm{rps}}^{(1)}, y_{\mathrm{rps}}^{(2)}, x_{\mathrm{rps}}^{(1)}, x_{\mathrm{rps}}^{(2)}\right)
$$

the change of coordinates relating the real/complex rps' to the cartesian coordinates. The real-analytic character of $\phi_{\mathrm{rps}}$ and hence of $\mathrm{H}_{\mathrm{rps}}:=\mathrm{H} \circ \phi_{\mathrm{rps}}$ on a region of phase space having the form $\widetilde{\mathcal{M}}_{\varepsilon_{0}} \times \mathbf{R}^{2}$, with $\widetilde{\mathcal{M}}_{\varepsilon_{0}}:=\widetilde{\mathcal{L}} \times \mathbf{T}^{2} \times B_{\varepsilon_{0}}^{6}(0)$ has been discussed in Refs. ${ }^{9,34}$.

In this paper, we are interested to look at the so-called "retrograde problem", whose motions live in a neighborhood of the singular manifold

$$
\mathcal{M}_{\pi}:=\left\{\mathrm{G}_{1}=\Lambda_{1} \text { or } \mathrm{G}_{2}=\Lambda_{2} \text { or } \mathrm{G}=\mathrm{G}_{1}-\mathrm{G}_{2} \text { or } \mathrm{Z}=\mathrm{G}\right\}
$$

It turns out that (see Proposition III.2) the one can find regular coordinates on a region of phase space $\mathcal{M}_{\varepsilon_{0}} \times \mathbf{R}^{2}$, where

$$
\mathcal{M}_{\varepsilon_{0}}:=\mathcal{L} \times \mathbf{T}^{2} \times B_{\varepsilon_{0}}^{6}(0)
$$

including a neighborhood of $\mathcal{M}_{\pi}$ and that the formulae of such regularizing coordinates are very simply related to the ones of the prograde case, as now we briefly discuss. We denote as

$$
\begin{gathered}
\operatorname{rps}_{\pi}:=(\Lambda, \lambda, \eta, \xi, p, q, P, Q)=\left(\Lambda_{1}, \Lambda_{2}, \lambda_{1}, \lambda_{2}, \eta_{1}, \eta_{2}, \xi_{1}, \xi_{2}, p, q, P, Q\right) \\
\operatorname{rps}_{\pi}^{\mathbf{C}}:=\left(\Lambda, \lambda, t, t^{*}, T, T^{*}\right)=\left(\Lambda_{1}, \Lambda_{2}, \lambda_{1}, \lambda_{2}, \tau_{1}, \tau_{2}, \tau_{3}, t_{1}^{*}, t_{2}^{*}, t_{3}^{*}, T, T^{*}\right)
\end{gathered}
$$

the real/complex coordinates which are defined via the formulae

$$
\left\{\begin{array} { l } 
{ \Lambda _ { 1 } = \Lambda _ { 1 } } \\
{ \Lambda _ { 2 } = \Lambda _ { 2 } } \\
{ t _ { 1 } = \sqrt { \Lambda _ { 1 } - \mathrm { G } _ { 1 } } e ^ { \mathrm { i } ( \gamma _ { 1 } + \gamma + \zeta ) } } \\
{ t _ { 2 } = - \mathrm { i } \sqrt { \Lambda _ { 2 } - \mathrm { G } _ { 2 } } e ^ { \mathrm { i } ( - \gamma _ { 2 } + \gamma + \zeta ) } } \\
{ t _ { 3 } = - \mathrm { i } \sqrt { \mathrm { G } - \mathrm { G } _ { 1 } + \mathrm { G } _ { 2 } } e ^ { \mathrm { i } ( \gamma + \zeta ) } } \\
{ T = \sqrt { \mathrm { G } - \mathrm { Z } } e ^ { \mathrm { i } \zeta } }
\end{array} \quad \left\{\begin{array}{l}
\lambda_{1}=\ell_{1}+\gamma_{1}+\gamma+\zeta \\
\lambda_{2}=\ell_{2}+\gamma_{2}-\gamma-\zeta \\
t_{1}^{*}=-\mathrm{i} \sqrt{\Lambda_{1}-\mathrm{G}_{1}} e^{-\mathrm{i}\left(\gamma_{1}+\gamma+\zeta\right)} \\
t_{2}^{*}=-\sqrt{\Lambda_{2}-\mathrm{G}_{2}} e^{-\mathrm{i}\left(-\gamma_{2}+\gamma+\zeta\right)} \\
t_{3}^{*}=-\sqrt{\mathrm{G}-\mathrm{G}_{1}+\mathrm{G}_{2}} e^{-\mathrm{i}(\gamma+\zeta)} \\
T^{*}=-\mathrm{i} \sqrt{\mathrm{G}-\mathrm{Z}} e^{-\mathrm{i} \zeta}
\end{array}\right.\right.
$$


and

$$
\begin{array}{llrr}
t_{1}:=\frac{\eta_{1}-\mathrm{i} \xi_{1}}{\sqrt{2}} & t_{2}:=\frac{\mathrm{i} \eta_{2}-\xi_{2}}{\sqrt{2}} & t_{3}:=\frac{\mathrm{i} p-q}{\sqrt{2}} \quad T:=\frac{P-\mathrm{i} Q}{\sqrt{2}} \\
t_{1}^{*}:=\frac{\eta_{1}+\mathrm{i} \xi_{1}}{\sqrt{2} \mathrm{i}} & t_{2}^{*}:=\frac{\mathrm{i} \eta_{2}+\xi_{2}}{\sqrt{2} \mathrm{i}} & t_{3}^{*}:=\frac{\mathrm{i} p+q}{\sqrt{2} \mathrm{i}} \quad T^{*}:=\frac{P+\mathrm{i} Q}{\sqrt{2} \mathrm{i}} .
\end{array}
$$

Observe that $\left\{\left(t_{1}, t_{2}, t_{3}, T, t_{1}^{*}, t_{2}^{*}, t_{3}^{*}, T^{*}\right)=(0,0,0,0,0,0,0,0)\right\}$ is the equation of the manifolds $\mathcal{M}_{\pi}$ 's. We let

$$
\begin{array}{ll}
\phi_{\mathrm{rps}_{\pi}}: & \operatorname{rps}_{\pi}=(\Lambda, \lambda, \eta, \xi, p, q, P, Q) \rightarrow\left(y_{\mathrm{rps}_{\pi}}^{(1)}, y_{\mathrm{rps}_{\pi}}^{(2)}, x_{\mathrm{rps}_{\pi}}^{(1)}, x_{\mathrm{rps}_{\pi}}^{(2)}\right) \\
\phi_{\mathrm{rps}_{\pi}^{\mathbf{C}}}^{\mathbf{C}}: & \operatorname{rps}_{\pi}^{\mathbf{C}}=\left(\Lambda, \lambda, t, t^{*}, T, T^{*}\right) \rightarrow\left(y_{\mathrm{rps}_{\pi}^{\mathbf{C}}}^{(1)}, y_{\mathrm{rps}_{\pi}^{\mathbf{C}}}^{(2)}, x_{\mathrm{rps}_{\pi}^{\mathbf{C}}}^{(1)}, x_{\mathrm{rps}_{\pi}^{\mathbf{C}}}^{(2)}\right)
\end{array}
$$

Let $\phi_{2}^{-}$be the involution

$$
\phi_{2}^{-}\left(\Lambda_{1}, \Lambda_{2}, \lambda_{1}, \lambda_{2}, t, t^{*}, T, T^{*}\right):=\left(\Lambda_{1},-\Lambda_{2}, \lambda_{1},-\lambda_{2}, t, t^{*}, T, T^{*}\right) .
$$

Then

Proposition III.1 $\phi_{\mathrm{rps}_{\pi}^{\mathrm{C}}}=\phi_{\mathrm{rps}} \mathrm{C} \circ \phi_{2}^{-}$.

Proposition III.1 (the proof of which can be found in Appendix A 1) is useful in order to derive the explicit expression of $\phi_{\mathrm{rps}_{\pi}}$ from the one of $\phi_{\mathrm{rps}_{\pi}}$, as done in Appendix A 1 e. The latter is needed to determine a analyticity domain for the Hamiltonian

$$
\begin{aligned}
\mathrm{H}_{\mathrm{rps}_{\pi}} & =\mathrm{H} \circ \phi_{\mathrm{rps}_{\pi}}=-\frac{\mathrm{m}_{1}^{3} \mathrm{M}_{1}^{2}}{2 \Lambda_{1}^{2}}-\frac{\mathrm{m}_{2}^{3} \mathrm{M}_{2}^{2}}{2 \Lambda_{2}^{2}}+\mu\left(\frac{y_{\mathrm{rps}_{\pi}}^{(1)} \cdot y_{\mathrm{rps}_{\pi}}^{(2)}}{m_{0}}-\frac{m_{1} m_{2}}{\left|x_{\mathrm{rps}_{\pi}}^{(1)}-x_{\mathrm{rps}_{\pi}}^{(2)}\right|}\right) \\
& =: \mathrm{h}_{\mathrm{k}}(\Lambda)+\mu f_{\mathrm{rps}_{\pi}}(\Lambda, \lambda, \eta, \xi, p, q)
\end{aligned}
$$

which we shall provide in the next section.

\section{A domain of analyticity for $\mathrm{H}_{\mathrm{rps}_{\pi}}$}

Let $\chi, \alpha_{-}, \alpha_{+}$, be pure numbers verifying

$$
\chi>1, \quad 0<\alpha_{-}<\alpha_{+}<1
$$

Let the masses $m_{0}, m_{1}, m_{2}$ and the number $\mu$ in (2)-(3) be chosen so that

$$
m_{2}<\frac{\sqrt{\alpha_{-}}}{2 \chi} m_{1}, \quad 0<\mu<\frac{3 m_{0}}{m_{1}-4 m_{2}}
$$


and let, for fixed $\Lambda_{-}, \Lambda_{+}$, with

$$
\begin{gathered}
0<\Lambda_{-}<\Lambda_{+}, \\
\mathcal{L}:=\left\{\Lambda=\left(\Lambda_{1}, \Lambda_{2}\right): \Lambda_{-}<\Lambda_{2}<\Lambda_{+}, \quad k_{-} \Lambda_{2}<\Lambda_{1}<k_{+} \Lambda_{2}\right\}
\end{gathered}
$$

where

$$
k_{ \pm}:=\frac{m_{1}}{m_{2}} \sqrt{\frac{m_{0}+\mu m_{2}}{m_{0}+\mu m_{1}} \alpha_{ \pm}} .
$$

We the take, for the coordinates $\operatorname{rps}_{\pi}=(\Lambda, \lambda, \eta, \xi, p, q, P, Q)$ in $(27)$ the domain $\mathcal{M}_{\varepsilon_{0}} \times$ $\{(0,0)\}$, where $\mathcal{M}_{\varepsilon_{0}}$ is as in $(23)$.

\section{Remark III.1}

* The cyclic pair $(P, Q)$ has been fixed, as in Refs. ${ }^{9,34}$, to $(0,0)$. This corresponds to fix the direction of the third axis of the prefixed reference frame parallel to the total angular momentum direction and has not influence on the dynamics.

* The bound in (31) for $m_{2}$ is not purely technical, but necessary in order to realize the ORC configuration, with arbitrarily small eccentricities and inclinations. Indeed, when the motions are planar, circular and orC, one has $\Lambda_{1}=\left|\mathrm{C}^{(1)}\right|>\left|\mathrm{C}^{(2)}\right|=\Lambda_{2}$, which implies, as discussed above, (31).

* Recalling that (by (3) and (7)) the semi-axes ratio $\alpha:=\frac{a_{1}}{a_{2}}$ is related to the ratio $\frac{\Lambda_{1}}{\Lambda_{2}}$ via

$$
\frac{\Lambda_{1}}{\Lambda_{2}}=\frac{\mathrm{m}_{1}}{\mathrm{~m}_{2}} \sqrt{\frac{\mathrm{M}_{1}}{\mathrm{M}_{2}} \alpha}=\frac{m_{1}}{m_{2}} \sqrt{\frac{m_{0}+\mu m_{2}}{m_{0}+\mu m_{1}} \alpha}
$$

and that, by definition of $\mathcal{L}, k_{-}<\frac{\Lambda_{1}}{\Lambda_{2}}<k_{+}$and using the definitions of $k_{ \pm}$in (34), we have

$$
\alpha_{-}<\alpha<\alpha_{+}
$$

Below, we shall choice $\alpha_{+}<\alpha_{s}$, where $\alpha_{s}, \mathcal{L}_{s}$ are as in $(44)$, so that we shall be able to use the results of the previous sections.

Proposition III.2 One can find $\varepsilon_{0}>0$, depending only on $\Lambda_{-}, \chi, \alpha_{+}$such that the function $\mathrm{H}_{\mathrm{rps}_{\pi}}$ in (29) is real-analytic for $(\Lambda, \lambda, \eta, \xi, p, q) \in \mathcal{M}_{\varepsilon_{0}}$, where $\mathcal{M}_{0}$ is as in (23), for all choices of $m_{0}, m_{1}, m_{2}, \alpha_{-}, \Lambda_{+}$and $\mu$ satisfying (30), (31) and (32).

We have to ensure that, on the domain $\mathcal{M}_{\varepsilon_{0}}$, the map $\phi_{\mathrm{rps}_{\pi}}$ discussed in Section A 1 e is realanalytic and collisions are excluded. Observe that, as a consequence of $(31), \frac{m_{1}}{m_{2}} \sqrt{\alpha_{-}}>2 \chi$, 
$\sqrt{\frac{m_{0}+\mu m_{2}}{m_{0}+\mu m_{1}}}>\frac{1}{2}$, whence $\chi<k_{-}<k_{+}$. This inequality implies, on $\mathcal{L},\left|\Lambda_{1}-\Lambda_{2}\right| \geq \Lambda_{-}(\chi-1)$ and therefore $\left|\Lambda_{1}-\Lambda_{2}\right| \geq \frac{1}{2} \Lambda_{-}(\chi-1)$ on a complex neighborhood of $\mathcal{L}$ depending on $\Lambda_{-}, \chi$. Therefore, the former common denominator of $c_{1}^{*}, c_{2}, c_{2}^{*}$ in (A18) well separated from zero as soon as $\varepsilon<\frac{1}{4} \sqrt{\Lambda_{-}(\chi-1)}$. The other denominators are treated in a similar way. The fact that the planar Kepler maps (A19) are analytic and that collisions are excluded on a complex neighborhood of $\mathcal{M}_{\varepsilon}$ depending only on $\Lambda_{-}, \alpha_{+}$and $\chi$ are classical facts and will be not discussed.

\section{D'Alembert rules}

The Hamiltonian $\mathrm{H}$ in (2) is invariant by rotations of the reference frame and reflections of the coordinates axes. These symmetries reflect on the Hamiltonian $\mathrm{H}_{\mathrm{rps}_{\pi}}$ in (29) and they will be named here "D'Alembert rules", by similarity with analogue relations holding when $\mathrm{H}$ is written in Poincaré coordinates, where they are given such name. A full analytical discussion may be found in Appendix A $1 \mathrm{f}$. In this section, we summarize their effect on the averaged perturbing function. More precisely, we focus on the system written in complex coordinates

$$
\mathrm{H}_{\mathrm{rps}}^{\mathbf{C}}\left(\Lambda, \lambda, t, t^{*}\right):=\mathrm{H} \circ \phi_{\mathrm{rps}_{\pi}^{\mathbf{C}}}\left(\Lambda, \lambda, t, t^{*}\right)=\mathrm{h}_{\mathrm{k}}\left(\Lambda_{1}, \Lambda_{2}\right)+\mu f_{\mathrm{rps}_{\pi}^{\mathbf{C}}}\left(\Lambda, \lambda, t, t^{*}\right)
$$

and we let

$$
f_{\mathrm{rps}}^{\mathrm{av}}\left(\Lambda, t, t^{*}\right):=\frac{1}{(2 \pi)^{2}} \int_{[0,2 \pi]^{2}} f_{\mathrm{rps}_{\pi}^{\mathrm{C}}}\left(\Lambda, \lambda, t, t^{*}\right) d \lambda .
$$

Let $c_{a, a^{*}}$ be the coefficients of the expansion of $f_{\mathrm{rps}_{\pi}^{\mathrm{C}}}^{\mathrm{av}}$ up to a prefixed order $m$

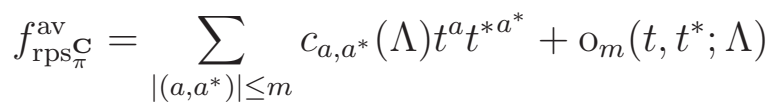

(which are well defined if the corresponding real coordinates vary in the domain $\mathcal{M}_{\varepsilon_{0}}$ as in Proposition III.2) then

$\left(\mathrm{s}_{1}\right)$ by $(\mathrm{A} 21), c_{a, a^{*}}(\Lambda) \neq 0 \Longrightarrow \sum_{i} a_{i}=\sum_{i} a_{i}^{*}$;

$\left(\mathrm{s}_{2}\right)$ by the latter identity in (A23) and previous item, $f_{\mathrm{rps}_{\pi}}^{\mathrm{av}}$ is even in $\left(t_{3}, t_{3}^{*}\right)$. Therefore, by the previous item, $f_{\mathrm{rps} \pi}^{\mathrm{av}}$ is even in $\left(t_{3}, t_{3}^{*}\right)$ and $\left(t_{1}, t_{2}, t_{1}^{*}, t_{2}^{*}\right)$ separately; 
$\left(\mathrm{s}_{3}\right)$ by the former identity in (A23) and the parity in $\left(t_{1}, t_{2}, t_{1}^{*}, t_{2}^{*}\right)$, the coefficients $c_{a, a^{*}}$ in (37) do not change letting

$$
\left(a_{1}, a_{2}, a_{1}^{*}, a_{2}^{*}\right) \rightarrow\left(a_{1}^{*}, a_{2}^{*}, a_{1}, a_{2}\right)
$$

and leaving $\left(a_{3}, a_{3}^{*}\right)$ unvaried;

$\left(\mathrm{s}_{4}\right)$ by the reality conditions $f_{\mathrm{rps} \pi}^{\mathrm{av}}=\left(f_{\mathrm{rps}_{\pi}}^{\mathrm{av}}\right)^{\mathrm{cc}}$,

$$
c_{a, a^{*}}=(-1)^{\frac{|a|+\left|a_{*}\right|}{2}}(-1)^{a_{2}+a_{2}^{*}+a_{3}+a_{3}^{*}} c_{a^{*}, a}
$$

as it follows using the following relations (implied by (26))

$$
\left(t_{1}, t_{1}^{*}\right)^{\mathrm{cc}}=\mathrm{i}\left(t_{1}^{*}, t_{1}\right), \quad\left(t_{j}, t_{j}^{*}\right)^{\mathrm{cc}}=-\mathrm{i}\left(t_{j}^{*}, t_{j}\right), \quad j=2,3
$$

into (37).

\section{Birkhoff theory in the partially reduced retrograde problem}

A remarkable consequence of $\left(\mathrm{s}_{1}\right)-\left(\mathrm{s}_{4}\right)$ is that (as already mentioned in the introduction), differently from what happens in the prograde problem, in the case of the retrograde one, it is not possible to guarantee a priori that the eigenvalues of the matrix $\sigma$ appearing in the second-order expansion

$$
f_{\mathrm{rps} \pi}^{\mathrm{av}}=C_{0}\left(\Lambda_{1}, \Lambda_{2}\right)+\mathrm{i} t_{h} \cdot \sigma(\Lambda) t^{*}+\mathrm{i} \varsigma(\Lambda) t_{3} t_{3}^{*}+\mathrm{O}_{4}\left(t, t^{*} ; \Lambda\right)
$$

where $t_{h}:=\left(t_{1}, t_{2}\right), t_{h}^{*}:=\left(t_{1}^{*}, t_{2}^{*}\right)$, are real. Indeed, the $\mathrm{s}_{1}-\mathrm{s}_{4}$ imply that the entries $\sigma_{i j}$ of $\sigma$ and $\varsigma$ in (39) verify

$$
\sigma_{11} \sigma_{22} \in \mathbf{R}, \quad \sigma_{12}=\sigma_{21} \in \mathrm{iR}, \quad \varsigma \in \mathbf{R}
$$

(in the case of the prograde problem, studied in $\operatorname{Refs}^{9,34}$, one has, correspondingly, that $\sigma$ is symmetric and real, because of the different form of the reality condition $\mathrm{s}_{4}$ ). The following result is therefore not trivial:

Proposition III.3 (Herman resonance, Birkhoff theory and symmetries in the partially reduced retrograde problem)

Let $\mathcal{L}$ be as in (33), $\varepsilon_{0}$ as in Proposition III.2. 
(i) For $\Lambda \in \mathcal{L}$, the matrix $\sigma(\Lambda)$ in (39) has two distinct eigenvalues $\sigma_{1}(\Lambda), \sigma_{2}(\Lambda)$ which, together with $\varsigma$, are real-analytic for all $\Lambda \in \mathcal{L}$. Moreover, for all $\Lambda \in \mathcal{L}$, one can find a a real-analytic vector matrix $\Omega: \Lambda \in \mathcal{L} \rightarrow \Omega(\Lambda) \in \mathbf{R}^{3}$, a symplectic, real-analytic $4 \times 4$ function matrix $V: \Lambda \in \mathcal{L} \rightarrow V(\Lambda)$ and a real-analytic, canonical transformation

$$
\begin{aligned}
\check{\phi}: \quad \check{\mathcal{M}}_{\check{\varepsilon}}:= & \mathcal{L} \times \mathbf{T}^{2} \times B_{\check{\varepsilon}}^{6}(0) \rightarrow \mathcal{M}_{\varepsilon_{0}} \\
& (\Lambda, \check{\lambda},(\check{\eta}, \check{\xi}), \check{p}, \check{q}) \rightarrow(\Lambda, \check{\lambda}+\check{\varphi}(\Lambda, \check{\eta}, \check{\xi}), V(\Lambda)(\check{\eta}, \check{\xi}), \check{p}, \check{q})
\end{aligned}
$$

such that, if

$$
\check{\mathrm{H}}:=\mathrm{H} \circ \check{\phi}=\mathrm{h}_{\mathrm{k}}(\Lambda)+\mu \check{f}(\Lambda, \check{\lambda}, \check{\eta}, \check{\xi}, \check{p}, \check{q})
$$

then

$$
\check{f}^{\mathrm{av}}=C_{0}(\Lambda)+\Omega(\Lambda) \cdot \check{\tau}+\mathrm{O}_{4}(\check{\eta}, \check{\xi}, \check{p}, \check{q} ; \Lambda)
$$

with

$$
\check{\tau}=\left(\frac{\check{\eta}_{1}^{2}+\check{\xi}_{1}^{2}}{2}, \frac{\check{\eta}_{2}^{2}+\check{\xi}_{2}^{2}}{2}, \frac{\check{p}^{2}+\check{q}^{2}}{2}\right)
$$

Moreover, for any prefixed $s \in \mathbf{N}$, one can find $\alpha_{s}$ such that, if

$$
\mathcal{L}_{s}:=\left\{\Lambda \in \mathcal{L}: \quad 0<\alpha<\alpha_{s}\right\}
$$

$$
\forall \Lambda \in \mathcal{L}_{s}, \quad k \in \mathbf{Z}^{3}, \quad 0<|k| \leq 2 s, \quad k \neq m(1,1,1), \quad m \in \mathbf{Z}: \quad \Omega(\Lambda) \cdot k \neq 0
$$

(ii) It is possible to find a real-analytic $3 \times 3$ matrix function $\mathrm{T}: \Lambda \in \mathcal{L}_{s} \rightarrow \mathrm{T}(\Lambda)$ and real-analytic functions $(\Lambda, \bar{\tau}) \in \mathcal{L}_{s} \times \mathbf{C}^{3} \rightarrow \mathcal{P}_{j}(\Lambda, \bar{\tau})$ with $\mathcal{P}_{j}(\Lambda, \bar{\tau})$ a polynomial of degree $j=3, \cdots$, s in $\bar{\tau}$ and a real-analytic and canonical transformation

$$
\begin{aligned}
\bar{\phi}: \quad \overline{\mathcal{M}}_{\bar{\varepsilon}}:=\mathcal{L}_{s} \times \mathbf{T}^{2} \times B_{\bar{\varepsilon}}^{6}(0) & \rightarrow \check{\mathcal{M}} \check{\varepsilon}=\mathcal{L}_{s} \times \mathbf{T}^{2} \times B_{\check{\varepsilon}}^{6}(0) \\
(\Lambda, \bar{\lambda}, \bar{\eta}, \bar{\xi}, \bar{p}, \bar{q}) & \rightarrow(\Lambda, \check{\lambda}, \check{\eta}, \check{\xi}, \check{p}, \check{q})=(\Lambda, \bar{\lambda}+\bar{\varphi}(\Lambda, \bar{\eta}, \bar{\xi}, \bar{p}, \bar{q}), \bar{B}(\bar{\eta}, \bar{\xi}, \bar{p}, \bar{q} ; \Lambda))
\end{aligned}
$$

such that, if

$$
\overline{\mathrm{H}}:=\check{\mathrm{H}} \circ \bar{\phi}=\mathrm{h}_{\mathrm{k}}(\Lambda)+\mu \bar{f}(\Lambda, \bar{\lambda}, \bar{\eta}, \bar{\xi}, \bar{p}, \bar{q}) \quad(\Lambda, \bar{\lambda}, \bar{\eta}, \bar{\xi}, \bar{p}, \bar{q}) \in \mathcal{L}_{2 s} \times \mathbf{T}^{2} \times B_{\bar{\varepsilon}}^{6}(0)
$$

then

$$
\bar{f}^{\mathrm{av}}=C_{0}(\Lambda)+\Omega \cdot \bar{\tau}+\frac{1}{2} \bar{\tau} \cdot \mathrm{T}(\Lambda) \bar{\tau}+\sum_{j=3}^{s} \overline{\mathcal{P}}(\bar{\tau} ; \Lambda)+\mathrm{O}_{2 s+2}(\bar{\eta}, \bar{\xi}, \bar{p}, \bar{q} ; \Lambda)
$$

where

$$
\bar{\tau}=\left(\frac{\bar{\eta}_{1}^{2}+\bar{\xi}_{1}^{2}}{2}, \frac{\bar{\eta}_{2}^{2}+\bar{\xi}_{2}^{2}}{2}, \frac{\bar{p}^{2}+\bar{q}^{2}}{2}\right)
$$


(iii) If $\widetilde{\Omega}\left(\Lambda_{1}, \Lambda_{2}\right)$ and $\widetilde{\mathrm{T}}\left(\Lambda_{1}, \Lambda_{2}\right)$ denote the first and second order Birkhoff invariants for the prograde problem with $n=2$ (denoted in Ref. ${ }^{9}$ (Proposition 7.1 and Equation (8.1)), respectively, as $(\sigma, \bar{\varsigma}), \tau)$ then $\Omega$, T are related to $\widetilde{\Omega}, \widetilde{\mathrm{T}}$ in via

$$
\Omega_{i}\left(\Lambda_{1}, \Lambda_{2}\right)=s_{i} \widetilde{\Omega}_{i}\left(\Lambda_{1},-\Lambda_{2}\right), \quad \mathrm{T}_{i j}\left(\Lambda_{1}, \Lambda_{2}\right)=s_{i} s_{j} \widetilde{\mathrm{T}}_{i j}\left(\Lambda_{1},-\Lambda_{2}\right) 1 \leq i \leq j \leq 3
$$

with $s_{1}=-s_{2}=-s_{3}=1$;

(iv) $\check{\phi}$ and $\breve{\phi}$ commute with $\mathcal{R}_{g}, \mathcal{R}_{3}^{-}$and $\mathcal{R}_{1 \leftrightarrow 2}$ in (A22) and (A24).

Remark III.2 The transformation $\check{\phi}$ preserves the function

$$
\mathrm{G}=\Lambda_{1}-\Lambda_{2}-\frac{\eta_{1}^{2}+\xi_{1}^{2}}{2}+\frac{\eta_{2}^{2}+\xi_{2}^{2}}{2}+\frac{p^{2}+q^{2}}{2}
$$

Differently from the prograde case, $V(\Lambda) \notin \mathrm{SO}(4)$.

Proof. (i) The transformation $\tilde{\phi}$ in Ref. $^{9}$ (Equations (7.20) and (7.22)) will be here denoted at

$$
\widehat{\phi}:(\Lambda, \widehat{\lambda}, \widehat{z}) \rightarrow(\Lambda, \widetilde{\lambda}, \widetilde{z})=(\Lambda, \widehat{\lambda}+\widehat{\psi}(\Lambda, \widehat{z}), \widehat{U}(\Lambda) \widehat{z})
$$

with $\widehat{z}=(\widehat{\eta}, \widehat{\xi}, \widehat{p}, \widehat{q}), \widetilde{z}=(\widetilde{\eta}, \widetilde{\xi}, \widetilde{p}, \widetilde{q}) . \widehat{\phi}$ projects as a transformation from

$$
\begin{aligned}
\widehat{\phi}^{\mathbf{C}}: \quad \mathcal{L} \times \mathbf{T}^{2} \times B_{\widehat{\varepsilon}}^{6}(0) & \rightarrow \mathcal{L} \times \mathbf{T}^{2} \times B_{\widehat{\varepsilon}}^{6}(0) \\
\left(\Lambda, \hat{\lambda},\left(\widehat{t}, \widehat{t}^{*}\right)\right) & \rightarrow\left(\Lambda, \widetilde{\lambda}, \widetilde{t}, \widetilde{t}^{*}\right)=\left(\Lambda, \widehat{\lambda}+\widehat{\psi}\left(\Lambda, \widehat{t}, \widehat{t}^{*}\right),\left(\widehat{U} \widehat{t}, \widehat{U} \widehat{t}^{*}\right)\right)
\end{aligned}
$$

where $\left(\widetilde{t}, \widetilde{t}^{*}\right),\left(\widehat{t}, \widehat{t}^{*}\right)$ are related to $\widetilde{z}=(\widetilde{\eta}, \widetilde{\xi}, \widetilde{p}, \widetilde{q}), \widehat{z}=(\widehat{\eta}, \widehat{\xi}, \widehat{p}, \widehat{q})$ at the same way as in (19), respectively. We then take $\check{\phi}^{\mathbf{C}}:=\phi_{2}^{-} \circ \widehat{\phi}^{\mathbf{C}} \circ \phi_{2}^{-}$, where $\phi_{2}^{-}$in as in (28). The transformation $\check{\phi}^{\mathbf{C}}$ is

$$
\begin{aligned}
\check{\phi}^{\mathbf{C}}: \quad \mathcal{L} \times \mathbf{T}^{2} \times B_{\check{\varepsilon}}^{6}(0) & \rightarrow \mathcal{L} \times \mathbf{T}^{2} \times B_{\check{\varepsilon}}^{6}(0) \\
\left(\Lambda, \check{\lambda}, \check{t}, \check{t}^{*}\right) & \rightarrow\left(\Lambda, \lambda, t, t^{*}\right)=\left(\Lambda, \check{\lambda}+\check{\psi}\left(\Lambda, \check{t}, \check{t}^{*}\right),\left(U \check{t}, U \check{t}^{*}\right)\right)
\end{aligned}
$$

with $\check{\psi}_{1}=\widehat{\psi}_{1}\left(\Lambda_{1},-\Lambda_{2}, \check{t}, \check{t}^{*}\right), \check{\psi}_{2}=-\widehat{\psi}_{2}\left(\Lambda_{1},-\Lambda_{2}, \check{t}, \check{t}^{*}\right), U\left(\Lambda_{1}, \Lambda_{2}\right)=\widehat{U}\left(\Lambda_{1},-\Lambda_{2}\right)$. Then $U$ is symmetric and verifies

$$
U^{-1}(\Lambda) \sigma(\Lambda) U(\Lambda)=\operatorname{diag}\left(\sigma_{1}(\Lambda), \sigma_{2}(\Lambda)\right)=: D(\Lambda)
$$

where $\sigma_{1}, \sigma_{2}$ are the eigenvalues of $\sigma$, with $\sigma_{i}\left(\Lambda_{1}, \Lambda_{2}\right)=\tilde{\sigma}_{i}\left(\Lambda_{1},-\Lambda_{2}\right)$. Observe that, while $U$ needs not to be real, since $\widehat{U} \in \mathrm{SO}(2), U$ verifies

$$
U^{\mathrm{t}}=U^{-1}, \quad \operatorname{det} U=1
$$


Note that $U$ is uniquely determined by conditions (52) and (53). Let $(\Lambda, \check{\lambda}, \check{\eta}, \check{\xi}, \check{p}, \check{q})$ be related to $\left(\Lambda, \check{\lambda}, \check{t}, \check{t}^{*}\right)$ via (26), and let $\check{\phi}$ be corresponding projection $\check{\phi}^{\mathrm{C}}$ as a transformation from $(\Lambda, \check{\lambda}, \check{\eta}, \check{\xi}, \check{p}, \check{q})$ to $(\Lambda, \lambda, \eta, \xi, p, q)$ of the form in (41), with

$$
\check{\varphi}(\Lambda, \check{\eta}, \check{\xi})=\check{\psi}(\Lambda, L(\check{\eta}, \check{\xi})) \quad V(\Lambda)=L^{-1} \operatorname{diag}(U(\Lambda), U(\Lambda)) L,
$$

where

$$
L=\left(\begin{array}{cccc}
\frac{1}{\sqrt{2}} & 0 & -\frac{\mathrm{i}}{\sqrt{2}} & 0 \\
0 & \frac{\mathrm{i}}{\sqrt{2}} & 0 & -\frac{1}{\sqrt{2}} \\
-\frac{\mathrm{i}}{\sqrt{2}} & 0 & \frac{1}{\sqrt{2}} & 0 \\
0 & \frac{1}{\sqrt{2}} & 0 & -\frac{\mathrm{i}}{\sqrt{2}}
\end{array}\right), \quad L^{-1}=\left(\begin{array}{cccc}
\frac{1}{\sqrt{2}} & 0 & \frac{\mathrm{i}}{\sqrt{2}} & 0 \\
0 & -\frac{\mathrm{i}}{\sqrt{2}} & 0 & \frac{1}{\sqrt{2}} \\
\frac{\mathrm{i}}{\sqrt{2}} & 0 & \frac{1}{\sqrt{2}} & 0 \\
0 & -\frac{1}{\sqrt{2}} & 0 & \frac{\mathrm{i}}{\sqrt{2}}
\end{array}\right)
$$

By construction, using also

$$
\frac{\check{\eta}_{1}^{2}+\check{\xi}_{1}^{2}}{2}=\mathrm{i} \check{t}_{1} \check{t}_{1}^{*}, \quad-\frac{\check{\eta}_{2}^{2}+\check{\xi}_{2}^{2}}{2}=\mathrm{i} \check{t}_{2} \check{t}_{2}^{*}, \quad-\frac{\check{p}^{2}+\check{q}^{2}}{2}=\mathrm{i} \check{t}_{3} \check{t}_{3}^{*}
$$

$\check{\phi}$ verifies (42) and (43), with $\sigma_{1}\left(\Lambda_{1}, \Lambda_{2}\right)=\widetilde{\sigma}_{1}\left(\Lambda_{1},-\Lambda_{2}\right), \sigma_{2}\left(\Lambda_{1}, \Lambda_{2}\right)=\widetilde{\sigma}\left(\Lambda_{1},-\Lambda_{2}\right), \varsigma\left(\Lambda_{1}, \Lambda_{2}\right)=$ $\widetilde{\varsigma}\left(\Lambda_{1},-\Lambda_{2}\right)$. We have to check that $\sigma_{1}, \sigma_{2}, \varsigma, \check{\varphi}$ and $V$ are real when their arguments are so. It follows from Ref. ${ }^{9}$ (Equations (B.1) and (B.2)) and Proposition III.1 that $f_{\mathrm{rps}_{\pi}^{\mathrm{C}}}^{\mathrm{av}}$ has the expression in (39), with $C\left(\Lambda_{1}, \Lambda_{2}\right)=\widetilde{C}\left(\Lambda_{1},-\Lambda_{2}\right)$ and

$$
\sigma\left(\Lambda_{1}, \Lambda_{2}\right)=\widetilde{\sigma}\left(\Lambda_{1},-\Lambda_{2}\right), \quad \varsigma\left(\Lambda_{1}, \Lambda_{2}\right)=\widetilde{\varsigma}\left(\Lambda_{1},-\Lambda_{2}\right) .
$$

Explicitly, using Ref. ${ }^{9}$ (Equation (B.1)), we find (according to (40))

$$
\sigma\left(\Lambda_{1}, \Lambda_{2}\right)=\left(\begin{array}{cc}
\frac{\mathrm{s}}{\Lambda_{1}} & -\mathrm{i} \frac{\widetilde{\mathrm{s}}}{\sqrt{\Lambda_{1} \Lambda_{2}}} \\
-\mathrm{i} \frac{\widetilde{\mathrm{s}}}{\sqrt{\Lambda_{1} \Lambda_{2}}} & -\frac{\mathrm{s}}{\Lambda_{2}}
\end{array}\right) \quad \varsigma(\Lambda)=-\left(\frac{1}{\Lambda_{1}}-\frac{1}{\Lambda_{2}}\right) \mathrm{s},
$$

with

$$
\mathrm{s}:=-m_{1} m_{2} \frac{\alpha}{2 a_{2}} b_{3 / 2}^{(1)}(\alpha) \quad \widetilde{\mathrm{s}}:=m_{1} m_{2} \frac{\alpha}{2 a_{2}} b_{3 / 2}^{(2)}(\alpha)
$$

with $\alpha, a_{2}$ as in (7), (35), and, as usual, $b_{s}^{(j)}(\alpha)$ 's being the Laplace coefficients, defined via the Fourier expansion

$$
\frac{1}{\left(1-2 \alpha \cos \theta+\alpha^{2}\right)^{s}}=\sum_{k \in \mathbf{Z}} b_{s}^{(k)}(\alpha) e^{\mathrm{i} k \theta} \quad \mathrm{i}:=\sqrt{(-1)} .
$$

The eigenvalues of $\sigma$ can be explicitly computed and they turn to be real. Indeed,

$$
\sigma_{1}, \sigma_{2}=\frac{\operatorname{tr} \sigma}{2} \pm \frac{1}{2} \sqrt{(\operatorname{tr} \sigma)^{2}-4 \operatorname{det} \sigma} .
$$


Since $\operatorname{tr} \sigma=\left(\frac{1}{\Lambda_{1}}-\frac{1}{\Lambda_{2}}\right)$ s is real, we have to check that the discriminant

$$
\Delta:=(\operatorname{tr} \sigma)^{2}-4 \operatorname{det} \sigma=\left(\frac{1}{\Lambda_{1}}-\frac{1}{\Lambda_{2}}\right)^{2} \mathrm{~s}^{2}+\frac{4}{\Lambda_{1} \Lambda_{2}}\left(\mathrm{~s}^{2}-\widetilde{\mathrm{s}}^{2}\right)
$$

is positive. Recalling that the Laplace coefficients verify

$$
b_{s}^{(j)}(\beta)>b_{s}^{(j+1)}(\beta) \quad \text { for all } \quad s>0, \quad j \in \mathbf{Z}, \quad 0<|\beta|<1,
$$

(see Ref. ${ }^{12}$ for a proof), one has

$$
\mathrm{s}^{2}-\widetilde{\mathrm{s}}^{2}=\left(m_{1} m_{2} \frac{\alpha}{a_{2}}\right)^{2}\left(\left(b_{3 / 2}^{(1)}(\alpha)\right)^{2}-\left(b_{3 / 2}^{(2)}(\alpha)\right)^{2}\right)>0 .
$$

and we have the assertion.

To prove the reality of $V(\Lambda)$, we first check that the matrix $U(\Lambda)$ has the form

$$
U(\Lambda)=\left(\begin{array}{cc}
\cosh x(\Lambda) & -\mathrm{i} \sinh x(\Lambda) \\
i \sinh x(\Lambda) & \cosh x(\Lambda)
\end{array}\right)
$$

with some $x=x(\Lambda) \in \mathbf{R}$. Let, for a given $2 \times 2$ matrix $A, \mathcal{C}(A)$ be defined via $\mathcal{C}(A)_{i i}=A_{i i}^{\text {cc }}$; $\mathcal{C}(A)_{i j}=-A_{i i}^{\text {cc }}$ for $i \neq j$. Since $\mathcal{C}(\sigma)=\sigma, \mathcal{C}(D)=D$ and $\mathcal{C}(A B)=\mathcal{C}(A) \mathcal{C}(B)$, applying $\mathcal{C}$ to the equality (52), we find $\mathcal{C}(U)=U$, which is equivalent to

$$
U_{i i}(\Lambda) \in \mathbf{R}, \quad U_{i j}(\Lambda) \in \mathrm{i} \mathbf{R} \quad \forall i \neq j=1,2 .
$$

Taking in count (53), (60), we find that $U$ has necessarily the form (59). Then, by direct computation, we find that $V(\Lambda)$ in (54) is given by

$$
V(\Lambda)=\operatorname{diag}(W(x(\Lambda)), W(-x(\Lambda))) \quad \text { with } \quad W(x)=\left(\begin{array}{cc}
\cosh x & \sinh x \\
\sinh x & \cosh x
\end{array}\right),
$$

We now check that $\varphi(\Lambda, \check{\eta}, \check{\xi})$ is real. We use the generating function of $\check{\phi}^{\mathbf{C}}$, which is

$$
S\left(\check{\Lambda}, \lambda, \check{t}, t^{*}\right)=\check{\Lambda} \lambda+t_{h}^{*} \cdot U \check{t}_{h}+\check{t}_{3} t_{3}^{*} .
$$

Then the function $\check{\psi}$ in (51) has the analytical form

$$
\psi\left(\Lambda, \check{t}_{h}, \check{t}_{h}^{*}\right)=\check{t}_{h}^{*} \cdot A(\Lambda) \check{t}_{h} \quad \text { where } \quad A(\Lambda):=U(\Lambda)^{-1} \partial_{\Lambda} U(\Lambda) .
$$

with $A(\Lambda)$ skew-symmetric, because of (53). Using (60) we find

$$
A(\Lambda)=\partial_{\Lambda} x(\Lambda)\left(\begin{array}{cc}
0 & -\mathrm{i} \\
\mathrm{i} & 0
\end{array}\right) \text {, }
$$


which gives

$$
\check{\psi}\left(\Lambda, \check{t}_{h}, \check{t}_{h}^{*}\right)=\mathrm{i} \partial_{\Lambda} x(\Lambda)\left(\check{t}_{1} \check{t}_{2}^{*}-\check{t}_{1}^{*} \check{t}_{2}\right)
$$

Then, by the first equation in (54) and (55),

$$
\check{\varphi}(\Lambda, \check{\eta}, \check{\xi})=\partial_{\Lambda} x(\Lambda)\left(\check{\eta}_{1} \check{\xi}_{2}+\check{\xi}_{1} \check{\eta}_{2}\right)
$$

is real on real arguments. The proof of (45) goes as in Ref. ${ }^{9}$ (Proof of Proposition 7.2) upon replacing $\sigma_{1}, \sigma_{2} \varsigma$ with

$\sigma_{1}=-\frac{3}{4 \Lambda_{1}} \frac{a_{1}^{2}}{a_{3}^{2}}+\mathrm{O}\left(\frac{a_{1}^{3}}{a_{2}^{4} \Lambda_{1}}\right) \quad \sigma_{2}=+\frac{3}{4 \Lambda_{2}} \frac{a_{1}^{2}}{a_{2}^{3}}+\mathrm{O}\left(\frac{a_{1}^{2}}{a_{2}^{3} \Lambda_{1}}\right), \quad \varsigma=\frac{3}{4} \frac{a_{1}^{2}}{a_{3}^{2}}+\mathrm{O}\left(\frac{a_{1}^{3}}{a_{2}^{4} \Lambda_{1}}\right)\left(\frac{1}{\Lambda_{1}}-\frac{1}{\Lambda_{2}}\right)$.

(ii) We denote as

$$
\begin{aligned}
\breve{\phi}: \quad \widetilde{\mathcal{L}} \times \mathbf{T}^{2} \times B_{\breve{\varepsilon}}^{6}(0) & \rightarrow \widetilde{\mathcal{L}} \times \mathbf{T}^{2} \times B_{\breve{\varepsilon}}^{6}(0) \\
(\Lambda, \breve{\lambda}, \breve{z}) & \rightarrow(\Lambda, \widehat{\lambda}, \widehat{z})=\left(\Lambda, \breve{\lambda}+\breve{\psi}(\Lambda, \breve{z}), \breve{B}^{\prime}(\Lambda, \breve{z})\right)
\end{aligned}
$$

with $\breve{z}=(\breve{\eta}, \breve{\xi}, \breve{p}, \breve{q}) \mathrm{m} \widehat{z}=(\widehat{\eta}, \widehat{\xi}, \widehat{p}, \widehat{q})$ the transformation in $\operatorname{Ref}^{9}$ (Equation (7.28)). As in the proof of Proposition III.3, we denote as $\left(\Lambda, \widehat{\lambda}, \widehat{t}, \hat{t}^{*}\right),\left(\Lambda, \breve{\lambda}, \breve{t}, \breve{t}^{*}\right)$ the coordinates related to $(\Lambda, \widehat{\lambda}, \widehat{z}),(\Lambda, \breve{\lambda}, \breve{z})$ via the relations in (19), respectively, and we let

$$
\begin{aligned}
\breve{\phi}^{\mathbf{C}}: \quad \tilde{\mathcal{L}} \times \mathbf{T}^{2} \times B_{\breve{\varepsilon}}^{6}(0) & \rightarrow \widetilde{\mathcal{L}} \times \mathbf{T}^{2} \times B_{\breve{\varepsilon}}^{6}(0) \\
\left(\Lambda, \breve{\lambda}, \breve{\eta}, \breve{t}, \breve{t}^{*}\right) & \rightarrow\left(\Lambda, \widehat{\lambda}, \widehat{t}, \widehat{t}^{*}\right)=\left(\Lambda, \breve{\lambda}+\breve{\psi}\left(\Lambda, \breve{\eta}, t, \breve{t}^{*}\right), \breve{B^{\prime}}\left(\breve{t}, \breve{t}^{*} ; \Lambda\right)\right)
\end{aligned}
$$

the transformation induced by $\breve{\phi}$ on such coordinates. Then, by definition,

$$
\breve{\mathrm{H}}^{\mathbf{C}}:=\mathrm{H} \circ \breve{\phi}^{\mathbf{C}}=\mathrm{h}_{\mathrm{k}}(\Lambda)+\mu \breve{f}^{\mathbf{C}}\left(\Lambda, \breve{\lambda}, \breve{t}, \breve{t}^{*}\right)
$$

with

$$
\breve{f}^{\mathbf{C}^{\text {av }}}=C_{0}(\Lambda)+\widetilde{\Omega} \cdot \breve{\tau}+\frac{1}{2} \breve{\tau} \cdot \breve{\mathrm{T}}(\Lambda) \breve{\tau}+\sum_{j=3}^{s} \breve{\mathcal{P}}(\breve{\tau} ; \Lambda)+\breve{\mathcal{R}}_{2 s+2}^{\mathbf{C}}\left(\Lambda, \breve{t}, \breve{t}^{*}\right)
$$

where

$$
\breve{\tau}_{1}=\frac{\breve{\eta}_{1}^{2}+\breve{\xi}_{1}^{2}}{2}=\mathrm{i} \breve{t}_{1} \breve{t}_{1}^{*}, \quad \breve{\tau}_{2}=\frac{\breve{\eta}_{2}^{2}+\breve{\xi}_{2}^{2}}{2}=\mathrm{i} \breve{t}_{2} \breve{t}_{2}^{*}, \quad \breve{\tau}_{3}=\frac{\breve{p}^{2}+\breve{q}_{1}^{2}}{2}=\mathrm{i} \breve{t}_{3} \breve{t}_{3}^{*}
$$

If $\phi_{2}^{-}$is as in (28), we let $\bar{\phi}^{\mathbf{C}}:=\phi_{2}^{-} \circ \breve{\phi}^{\mathbf{C}} \circ \phi^{\mathbf{C}}$ and $\overline{\mathrm{H}}^{\mathbf{C}}:=\check{\mathrm{H}} \circ \bar{\phi}^{\mathbf{C}}$.

Then $\bar{\phi}^{\mathrm{C}}$ and $\overline{\mathrm{H}}$ have the form

$$
\begin{aligned}
\bar{\phi}^{\mathbf{C}}: & \mathcal{L}_{s} \times \mathbf{T}^{2} \times B_{\bar{\varepsilon}}^{6}(0) \rightarrow \mathcal{L}_{s} \times \mathbf{T}^{2} \times B \frac{6}{\bar{\varepsilon}}(0) \\
& \left(\Lambda, \bar{\lambda}, \bar{\eta}, \bar{t}, \bar{t}^{*}\right) \rightarrow\left(\Lambda, \check{\lambda}, \check{t}, \check{t}^{*}\right)=\left(\Lambda, \bar{\lambda}+\bar{\psi}\left(\Lambda, \bar{\eta}, t, \bar{t}^{*}\right), \bar{B}^{\prime}\left(\bar{t}, \bar{t}^{*} ; \Lambda\right)\right) \\
& \overline{\mathrm{H}}^{\mathrm{C}}=\mathrm{H} \circ \bar{\phi}^{\mathrm{C}}=\mathrm{h}_{\mathrm{k}}(\Lambda)+\mu \bar{f}^{\mathrm{C}}\left(\Lambda, \bar{\lambda}, \bar{t}, \bar{t}^{*}\right)
\end{aligned}
$$


with

$$
\bar{f}^{\mathrm{Cav}}=\bar{C}_{0}(\Lambda)+\bar{\Omega} \cdot \bar{\tau}+\frac{1}{2} \bar{\tau} \cdot \overline{\mathrm{T}}(\Lambda) \bar{\tau}+\sum_{j=3}^{s} \overline{\mathcal{P}}(\bar{\tau} ; \Lambda)+\overline{\mathcal{R}}_{2 s+2}^{\mathrm{C}}\left(\Lambda, \bar{t}, \bar{t}^{*}\right)
$$

where $\bar{C}_{0}\left(\Lambda_{1}, \Lambda_{2}\right)=C_{0}\left(\Lambda_{1},-\Lambda_{2}\right), \bar{\Omega}\left(\Lambda_{1}, \Lambda_{2}\right)=\widetilde{\Omega}\left(\Lambda_{1},-\Lambda_{2}\right)=\left(\sigma_{1}, \sigma_{2}, \varsigma\right)$,

$$
\bar{\tau}_{1}=\mathrm{i} \bar{t}_{1} \bar{t}_{1}^{*}, \quad \bar{\tau}_{2}=\mathrm{i} \bar{t}_{2} \bar{t}_{2}^{*}, \quad \bar{\tau}_{3}=-\mathrm{i} \bar{t}_{3} \bar{t}_{3}^{*}
$$

and $\overline{\mathrm{T}}\left(\Lambda_{1}, \Lambda_{2}\right)=\breve{\mathrm{T}}\left(\Lambda_{1},-\Lambda_{2}\right)$. Now, if $(\Lambda, \bar{\lambda}, \bar{\eta}, \bar{\xi}, \bar{p}, \bar{q})$ are related to $\left(\Lambda, \bar{\lambda}, \bar{t}, \bar{t}^{*}\right)$ via the relations in $(26), \bar{\phi}^{\mathrm{C}}$, induces a transformation from $(\Lambda, \bar{\lambda}, \bar{\eta}, \bar{\xi}, \bar{p}, \bar{q})$ to $(\Lambda, \check{\lambda}, \check{\eta}, \check{\xi}, \check{p}, \check{q})$ having the form

$$
\begin{aligned}
\bar{\phi}: & \mathcal{L}_{s} \times \mathbf{T}^{2} \times B_{\bar{\varepsilon}}^{6}(0) \rightarrow \mathcal{L}_{s} \times \mathbf{T}^{2} \times B_{\bar{\varepsilon}}^{6}(0) \\
& (\Lambda, \bar{\lambda}, \bar{\eta}, \bar{\xi}, \bar{p}, \bar{q}) \rightarrow(\Lambda, \check{\lambda}, \check{\eta}, \check{\xi}, \check{p}, \check{q})=(\Lambda, \bar{\lambda}+\bar{\varphi}(\Lambda, \bar{\eta}, \bar{\xi}, \bar{p}, \bar{q}), \bar{B}(\bar{\eta}, \bar{\xi}, \bar{p}, \bar{q} ; \Lambda)) \\
& \overline{\mathrm{H}}=\mathrm{H} \circ \bar{\phi}=\mathrm{h}_{\mathrm{k}}(\Lambda)+\mu \bar{f}(\Lambda, \bar{\eta}, \bar{\xi}, \bar{p}, \bar{q})
\end{aligned}
$$

with

$$
\bar{f}^{\mathrm{av}}=\bar{C}_{0}(\Lambda)+\Omega \cdot \tau+\frac{1}{2} \tau \cdot \mathrm{T}(\Lambda) \tau+\sum_{j=3}^{s} \overline{\mathcal{P}}(\tau ; \Lambda)+\overline{\mathcal{R}}_{2 s+2}(\Lambda, \bar{\eta}, \bar{\xi}, \bar{p}, \bar{q})
$$

with

$$
\tau_{1}=\bar{\tau}_{1}=\frac{\bar{\eta}_{1}^{2}+\bar{\xi}_{1}^{2}}{2}, \quad \tau_{2}=-\bar{\tau}_{2}=\frac{\bar{\eta}_{2}^{2}+\bar{\xi}_{2}^{2}}{2}, \quad \tau_{3}=-\bar{\tau}_{3}=\frac{\bar{p}^{2}+\bar{q}^{2}}{2}
$$

and $\Omega, \mathrm{T}$ as in (48). The proof that $\bar{\phi}$ is real-analytic in $\overline{\mathcal{M}}_{\bar{\varepsilon}}$ is standard and therefore is omitted.

(iii) follows from (i) and (ii). (iv) is analogous to Refs. ${ }^{8,9}$.

\section{Birkhoff theory the fully reduced retrograde problem}

The construction of the Birkhoff normal form for the averaged, fully reduced system in the case of the prograde problem in has been discussed in Ref. ${ }^{9}$ (Sections 9 and 10). We recall the basic steps:

* Passage to a set of canonical coordinates on the manifold with constant $\mathrm{G}=|\mathrm{C}|$. This is accomplished via a transformation $\hat{\phi}$ described in Ref. ${ }^{9}$ (Section 9) which conjugates a "Birkhoff-normalized partially reduced system" $\breve{\mathcal{H}}$ discussed in Ref. ${ }^{9}$ (Equations (7.28)-(7.30)) to a fully reduced system $\hat{\mathcal{H}}_{\mathrm{G}}$, as described in Ref. ${ }^{9}$ (Remark 9.1); 


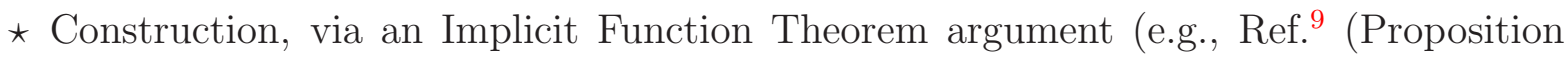
10.1)) of a canonical transformation $\check{\phi}$ which conjugates $\hat{\mathcal{H}}_{\mathrm{G}}$ to $\check{\mathcal{H}}_{\mathrm{G}}$, where the average $\check{\mathcal{H}}_{G}$ with respect the the angles $\check{\lambda}$ appearing in Ref. ${ }^{9}$ (Equation (10.10)) is in Birkhoff normal form of order 4 .

In the case of the retrograde problem, one can mimic the procedure above, or, equivalently, exploit the results of Ref. ${ }^{9}$ via Proposition III.1. Namely, if $\hat{\phi}^{\mathbf{C}}:\left(\Lambda, G, \hat{\lambda}, \hat{\mathrm{g}}, \hat{t}, \hat{t}^{*}\right) \rightarrow$ $\left(\Lambda, \breve{\lambda}, \breve{t}, \breve{t}^{*}\right), \check{\phi}^{\mathrm{C}}: \quad\left(\Lambda, \mathrm{G}, \check{\lambda}, \check{\mathrm{g}}, \check{t}, \breve{t}^{*}\right) \rightarrow\left(\Lambda, \mathrm{G}, \hat{\lambda}, \hat{\mathrm{g}}, \hat{t}, \hat{t}^{*}\right)$ denote the transformations induced by the transformations $\check{\phi}:(\Lambda, \mathrm{G}, \hat{\lambda}, \hat{\mathrm{g}}, \hat{\eta}, \hat{\xi}) \rightarrow(\Lambda, \breve{\lambda}, \breve{\eta}, \breve{\xi}, \breve{p}, \breve{q}), \check{\phi}: \quad(\Lambda, \mathrm{G}, \check{\lambda}, \check{\mathrm{g}}, \check{\eta}, \check{\xi}) \rightarrow$ $(\Lambda, \mathrm{G}, \hat{\lambda}, \hat{\mathrm{g}}, \hat{\eta}, \hat{\xi})$ of Ref. ${ }^{9}$ on the complex coordinates related to the respective real coordinates via relations to (19); $\phi_{2}^{-}$is as in (28); $\dot{\phi}^{\mathbf{C}}:=\phi_{2}^{-} \circ \hat{\phi}^{\mathbf{C}} \circ \check{\phi}^{\mathbf{C}} \circ \phi_{2}^{-}:\left(\Lambda, \dot{\lambda}, \dot{t}, \dot{t}^{*}\right) \rightarrow\left(\Lambda, \bar{\lambda}, \bar{t}, \bar{t}^{*}\right)$ then the transformation $\dot{\phi}:(\Lambda, \mathrm{G}, \dot{\lambda}, \dot{\gamma}, \dot{\eta}, \dot{\xi}) \rightarrow(\Lambda, \bar{\lambda}, \bar{\eta}, \bar{\xi}, \bar{p}, \bar{q})$ induced by $\dot{\phi}^{\mathrm{C}}$, such in a way that $(\Lambda, \mathrm{G}, \dot{\lambda}, \dot{\gamma}, \dot{\eta}, \dot{\xi}),(\Lambda, \bar{\lambda}, \bar{\eta}, \bar{\xi}, \bar{p}, \bar{q})$ are related to $\left(\Lambda, \dot{\lambda}, \dot{t}, \dot{t}^{*}\right),\left(\Lambda, \bar{\lambda}, \bar{t}, \bar{t}^{*}\right)$, respectively, via relations (26), turns to be real on a real domain and makes the following proposition true.

Proposition III.4 (BNF for the fully reduced retrograde problem) Fix $4 \leq s \in$ N. There exist $0<\dot{\varepsilon}_{1}<\frac{\dot{\varepsilon}_{2}}{4}$ such that, if

$$
\begin{aligned}
\dot{\mathcal{L}}_{s}:= & \left\{(\Lambda, G)=\left(\Lambda_{1}, \Lambda_{2}, \mathrm{G}\right) \in \mathcal{L}_{s} \times \mathbf{R}_{+}: \Lambda_{1}>\Lambda_{2}, \quad \mathrm{G}<\Lambda_{1}-\Lambda_{2}\right. \\
& \left.\varrho(\Lambda, \mathrm{G}):=\sqrt{2\left(\Lambda_{1}-\Lambda_{2}-\mathrm{G}\right)} \in\left(4 \dot{\varepsilon}_{1}, \dot{\varepsilon}_{2}\right)\right\} \\
& \dot{\mathcal{M}}_{\dot{\varepsilon}}:=\dot{\mathcal{L}}_{s} \times \mathbf{T}^{3} \times B_{\dot{\varepsilon}}^{4}(0)
\end{aligned}
$$

one can find a real-analytic and symplectic transformation

$$
\begin{aligned}
\dot{\phi}: \quad \dot{\mathcal{M}}_{\dot{\varepsilon}_{1}}=\dot{\mathcal{L}}_{s} \times \mathbf{T}^{3} \times B_{\dot{\varepsilon}_{1}}^{4}(0) & \rightarrow \overline{\mathcal{M}}_{\bar{\varepsilon}}:=\mathcal{L} \times \mathbf{T}^{2} \times B_{\bar{\varepsilon}}^{6}(0) \\
(\Lambda, \mathrm{G}, \dot{\lambda}, \dot{\gamma}, \dot{\eta}, \dot{\xi}) & \rightarrow(\Lambda, \dot{\lambda}+\dot{\varphi}(\Lambda, \dot{\eta}, \dot{\xi}), \dot{B}(\dot{\eta}, \dot{\xi} ; \Lambda))
\end{aligned}
$$

such that

$$
\dot{\mathrm{H}}_{\mathrm{G}}(\Lambda, \dot{\lambda}, \dot{\eta}, \dot{\xi})=\overline{\mathrm{H}} \circ \dot{\phi}(\Lambda, \dot{\lambda}, \dot{\eta}, \dot{\xi} ; \mathrm{G})=h_{\mathrm{k}}(\Lambda)+\mu \dot{f}(\Lambda, \dot{\lambda}, \dot{\eta}, \dot{\xi} ; \mathrm{G})
$$

is $\dot{\gamma}$-independent, and $\dot{f}$ av $(\Lambda, \dot{\eta}, \dot{\xi} ; \mathrm{G}):=\frac{1}{4 \pi^{2}} \int_{\mathbf{T}^{2}} \dot{f}(\Lambda, \dot{\lambda}, \dot{\eta}, \dot{\xi} ; \mathrm{G}) d \dot{\lambda}$ takes the form

$$
\begin{aligned}
\dot{f}^{\mathrm{av}}(\Lambda, \dot{\eta}, \dot{\xi} ; \mathrm{G}) & =\dot{C}(\Lambda ; \mathrm{G})+\dot{\Omega}(\Lambda ; \mathrm{G}) \cdot \dot{\tau}+\frac{1}{2} \dot{\tau} \dot{\mathrm{T}}(\Lambda ; \mathrm{G}) \cdot \dot{\tau}+\sum_{j=3}^{s} \dot{\mathcal{P}}_{j}(\dot{\tau} ; \Lambda, \mathrm{G}) \\
& +\mathrm{O}_{2 s+1}(\Lambda, \dot{\eta}, \dot{\xi} ; \mathrm{G})
\end{aligned}
$$


where $\dot{\tau}_{i}:=\frac{\dot{\eta}_{i}^{2}+\dot{\xi}_{i}^{2}}{2}$, and

$$
\begin{aligned}
& \dot{\Omega}_{i}\left(\Lambda_{1}, \Lambda_{2}\right)=s_{i} \hat{\Omega}_{i}\left(\Lambda_{1},-\Lambda_{2}\right) \\
& \dot{\mathrm{T}}_{i j}\left(\Lambda_{1}, \Lambda_{2} ; \mathrm{G}\right)=s_{i} s_{j} \hat{\mathrm{T}}_{i j}\left(\Lambda_{1},-\Lambda_{2}\right)+\mathrm{O}\left(\dot{\varepsilon}_{2}^{2}\right), 1 \leq i \leq j \leq 2
\end{aligned}
$$

with $s_{1}=-s_{2}=1$ and

$$
\begin{aligned}
& \hat{\Omega}_{i}\left(\Lambda_{1}, \Lambda_{2}\right)=\breve{\Omega}_{i}\left(\Lambda_{1}, \Lambda_{2}\right)-\breve{\Omega}_{3}\left(\Lambda_{1}, \Lambda_{2}\right) \\
& \hat{\mathrm{T}}_{i j}\left(\Lambda_{1}, \Lambda_{2}\right):=\breve{\mathrm{T}}_{i j}\left(\Lambda_{1}, \Lambda_{2}\right)-\breve{\mathrm{T}}_{i 3}\left(\Lambda_{1}, \Lambda_{2}\right)-\breve{\mathrm{T}}_{3 j}\left(\Lambda_{1}, \Lambda_{2}\right)+\breve{\mathrm{T}}_{33}\left(\Lambda_{1}, \Lambda_{2}\right) .
\end{aligned}
$$

Remark III.3 The analytical expression $\dot{\Omega}_{1}\left(\Lambda_{1}, \Lambda_{2}\right), \dot{\Omega}_{2}\left(\Lambda_{1}, \Lambda_{2}\right)$ which is available accordingly to (68) and Ref. ${ }^{9}$ (Equations (7.1), (7.5) with $n=2$ )

$$
\dot{\Omega}_{1}=-\frac{3}{4} m_{1} m_{2} \frac{a_{1}^{2}}{a_{2}^{3}}\left(\frac{2}{\Lambda_{1}}-\frac{1}{\Lambda_{2}}\right)\left(1+\mathrm{O}\left(\frac{a_{1}^{2}}{a_{2}^{2}}\right)\right) \quad \dot{\Omega}_{2}=\frac{3}{4} m_{1} m_{2} \frac{a_{1}^{2}}{a_{2}^{3}}\left(\frac{1}{\Lambda_{1}}-\frac{2}{\Lambda_{2}}\right)\left(1+\mathrm{O}\left(\frac{a_{1}^{2}}{a_{2}^{2}}\right)\right)
$$

shows that the $\dot{\Omega}_{i}$ 's do not satisfy, identically, linear combinations up to any prefixed order in $\dot{\mathcal{L}}_{s, \mathrm{G}}$. This is not used in the paper, but is useful to be known if one wants to obtain a stronger result concerning the measure of the invariant set (Ref. ${ }^{7}$ (Theorem 1.2))

\section{Torsion}

Define, for a fixed $\mathrm{G} \in \mathbf{R}$,

$$
\mathcal{L}_{s, \mathrm{G}}:=\left\{\left(\Lambda_{1}, \Lambda_{2}\right):\left(\Lambda_{1}, \Lambda_{2}, \mathrm{G}\right) \in \dot{\mathcal{L}}_{s}\right\}
$$

where $\dot{\mathcal{L}}_{s}$ is as in (44). In this section, we first compute the the matrix $\dot{\mathrm{T}}$ in (67) and next we check its non-singularity on $\dot{\mathcal{L}}_{s, \mathrm{G}}$. We proceed in three steps.

a. Computation of $\breve{\mathrm{T}}$ Note that we cannot use the asymptotics of the matrix $\breve{\mathrm{T}}$ given in Ref. ${ }^{9}$ (Equation (8.6)), because that was obained for $\Lambda_{1} \ll \Lambda_{2}$, while, for the purposes of the paper, we need $\Lambda_{1}>\Lambda_{2}$.

By (48), we evaluate the $\breve{\mathrm{T}}_{i j}\left(\Lambda_{1}, \Lambda_{2}\right)$ 's first. According to Refs. ${ }^{9,34}$, they have the form (neglecting to write the arguments)

$$
\begin{aligned}
\breve{\mathrm{T}}_{11}=\frac{4 m_{1} m_{2}}{\left(1+d^{2}\right)^{2}}[ & \frac{\mathrm{r}_{1}\left(a_{1}, a_{2}\right)}{\Lambda_{1}^{2}}+\frac{d^{4} \mathrm{r}_{1}\left(a_{2}, a_{1}\right)}{\Lambda_{2}^{2}}+\frac{2 d^{2} \mathrm{r}_{2}\left(a_{1}, a_{2}\right)}{\Lambda_{1} \Lambda_{2}} \\
& \left.-\frac{2 d \mathrm{r}_{3}\left(a_{1}, a_{2}\right)}{\Lambda_{1} \sqrt{\Lambda_{1} \Lambda_{2}}}-\frac{2 d^{3} \mathrm{r}_{3}\left(a_{2}, a_{1}\right)}{\Lambda_{2} \sqrt{\Lambda_{1} \Lambda_{2}}}+\frac{d^{2} \mathrm{r}_{4}\left(a_{1}, a_{2}\right)}{\Lambda_{1} \Lambda_{2}}\right]
\end{aligned}
$$




$$
\begin{aligned}
& \breve{\mathrm{T}}_{12}=\frac{4 m_{1} m_{2}}{\left(1+d^{2}\right)^{2}}[ \frac{\left(1-d^{2}\right)^{2} \mathrm{r}_{2}\left(a_{1}, a_{2}\right)}{\Lambda_{1} \Lambda_{2}}+\frac{2 d^{2} \mathrm{r}_{1}\left(a_{1}, a_{2}\right)}{\Lambda_{1}^{2}}+\frac{2 d^{2} \mathrm{r}_{1}\left(a_{2}, a_{1}\right)}{\Lambda_{2}^{2}} \\
&\left.+\frac{2 d\left(1-d^{2}\right) \mathrm{r}_{3}\left(a_{1}, a_{2}\right)}{\Lambda_{1} \sqrt{\Lambda_{1} \Lambda_{2}}}-\frac{2 d\left(1-d^{2}\right) \mathrm{r}_{3}\left(a_{2}, a_{1}\right)}{\Lambda_{2} \sqrt{\Lambda_{1} \Lambda_{2}}}+\frac{\left(1-6 d^{2}+d^{4}\right) \mathrm{r}_{4}\left(a_{1}, a_{2}\right)}{4 \Lambda_{1} \Lambda_{2}}\right] \\
& \breve{\mathrm{T}}_{22}= \frac{4 m_{1} m_{2}}{\left(1+d^{2}\right)^{2}}\left[\frac{\mathrm{r}_{1}\left(a_{2}, a_{1}\right)}{\Lambda_{2}^{2}}+\frac{d^{4} \mathrm{r}_{1}\left(a_{1}, a_{2}\right)}{\Lambda_{1}^{2}}+\frac{2 d^{2} \mathrm{r}_{2}\left(a_{1}, a_{2}\right)}{\Lambda_{1} \Lambda_{2}}\right. \\
&-\frac{2 d \mathrm{r}_{3}\left(a_{2}, a_{1}\right)}{\Lambda_{2} \sqrt{\Lambda_{1} \Lambda_{2}}}-\frac{2 d^{3} \mathrm{r}_{3}\left(a_{1}, a_{2}\right)}{\left.\Lambda_{1} \sqrt{\Lambda_{1} \Lambda_{2}}+\frac{d^{2} \mathrm{r}_{4}\left(a_{1}, a_{2}\right)}{\Lambda_{1} \Lambda_{2}}\right]} \\
& \breve{\mathrm{T}}_{13}=\frac{m_{1} m_{2}}{1+d^{2}}\left[\frac{1}{\Lambda_{1}}\left(\frac{1}{\Lambda_{1}}+\frac{1}{\Lambda_{2}}\right)\left(\mathrm{s}_{1}\left(a_{1}, a_{2}\right)+\mathrm{s}_{1}^{*}\left(a_{1}, a_{2}\right)\right)-\left(\frac{1}{\Lambda_{1}^{2}}+\frac{d^{2}}{\Lambda_{2}^{2}}\right) C_{1}\left(a_{1}, a_{2}\right)\right. \\
& \quad-\frac{d}{\sqrt{\Lambda_{1} \Lambda_{2}}}\left(\frac{1}{\Lambda_{1}}+\frac{1}{\Lambda_{2}}\right)\left(\mathrm{s}_{2}\left(a_{1}, a_{2}\right)+\mathrm{s}_{2}^{*}\left(a_{1}, a_{2}\right)\right) \\
&\left.\quad+\frac{d^{2}}{\Lambda_{2}}\left(\frac{1}{\Lambda_{1}}+\frac{1}{\Lambda_{2}}\right)\left(\mathrm{s}_{1}\left(a_{2}, a_{1}\right)+\mathrm{s}_{1}^{*}\left(a_{2}, a_{1}\right)\right)\right] \\
& \breve{\mathrm{T}}_{23}=\frac{m_{1} m_{2}}{1+d^{2}}\left[\frac{1}{\Lambda_{2}}\left(\frac{1}{\Lambda_{1}}+\frac{1}{\Lambda_{2}}\right)\left(\mathrm{s}_{1}\left(a_{2}, a_{1}\right)+\mathrm{s}_{1}^{*}\left(a_{2}, a_{1}\right)\right)-\left(\frac{1}{\Lambda_{2}^{2}}+\frac{d^{2}}{\Lambda_{1}^{2}}\right) C_{1}\left(a_{1}, a_{2}\right)\right. \\
& \quad+\frac{d}{\sqrt{\Lambda_{1} \Lambda_{2}}}\left(\frac{1}{\Lambda_{1}}+\frac{1}{\Lambda_{2}}\right)\left(\mathrm{s}_{2}\left(a_{1}, a_{2}\right)+\mathrm{s}_{2}^{*}\left(a_{1}, a_{2}\right)\right) \\
&\left.\quad+\frac{d^{2}}{\Lambda_{1}}\left(\frac{1}{\Lambda_{1}}+\frac{1}{\Lambda_{2}}\right)\left(\mathrm{s}_{1}\left(a_{1}, a_{2}\right)+\mathrm{s}_{1}^{*}\left(a_{1}, a_{2}\right)\right)\right] \\
& \breve{\mathrm{T}}_{33}=4 m_{1} m_{2}\left[\mathrm{r}_{1}^{*}\left(a_{1}, a_{2}\right)\left(\frac{1}{\Lambda_{1}}+\frac{1}{\Lambda_{2}}\right)^{2}+\frac{C_{1}\left(a_{1}, a_{2}\right)}{4 \Lambda_{1} \Lambda_{2}}\right]
\end{aligned}
$$

where $d$ depends on $\Lambda_{1}, \Lambda_{2}, a_{1}, a_{2}$, while $C_{1}, \mathrm{r}_{1}, \mathrm{r}_{2} \mathrm{r}_{3}, \mathrm{r}_{4}, \mathrm{r}_{1}^{*}, \mathrm{r}_{2}^{*}, \mathrm{~s}_{1}, \mathrm{~s}_{2}, \mathrm{~s}_{1}^{*}, \mathrm{~s}_{2}^{*}$ are functions of $a_{1}, a_{2}$ only (in turn related to $\Lambda_{1}, \Lambda_{2}$ via $(7)$ ), expressed in terms of the Laplace coefficients. Moreover, it follows from Ref. ${ }^{34}$ (Proof of Proosition 5.2) that

$$
d=d\left(\frac{\sqrt{\Lambda_{1} \Lambda_{2}}}{\left|\Lambda_{1}-\Lambda_{2}\right|} b(\alpha)\right)
$$

with

$$
d(x)=\frac{x}{\sqrt{1+x^{2}}+1}, \quad b(\alpha)=4 \frac{b_{3 / 2}^{(2)}(\alpha)}{b_{3 / 2}^{(1)}(\alpha)}=\mathrm{O}(\alpha)
$$

We recall that $d$ is defined so that the matrix $\widehat{U}$ in $(50)$ is

$$
\left(\begin{array}{cc}
1 & -\frac{d}{\sqrt{1+d^{2}}} \\
\frac{d}{\sqrt{1+d^{2}}} & 1
\end{array}\right)
$$


As in $\operatorname{Refs}^{9}{ }^{9,34}$, we shall use the expansions of the functions above in terms of $\alpha$

$$
\begin{array}{ll}
\mathrm{r}_{1}\left(a_{1}, a_{2}\right)=\frac{3}{16 a_{2}}\left(\alpha^{2}+\mathrm{O}\left(\alpha^{4}\right)\right) & \mathrm{r}_{1}\left(a_{2}, a_{1}\right)=-\frac{3}{4 a_{2}}\left(\alpha^{2}+\mathrm{O}\left(\alpha^{4}\right)\right) \\
\mathrm{r}_{2}\left(a_{1}, a_{2}\right)=\mathrm{r}_{2}\left(a_{2}, a_{1}\right)=-\frac{9}{16 a_{2}}\left(\alpha^{2}+\mathrm{O}\left(\alpha^{4}\right)\right) & \mathrm{r}_{3}\left(a_{1}, a_{2}\right)=\mathrm{O}\left(\frac{\alpha^{3}}{a_{2}}\right)=\mathrm{r}_{3}\left(a_{2}, a_{1}\right) \\
\mathrm{r}_{4}\left(a_{1}, a_{2}\right)=\mathrm{O}\left(\frac{\alpha^{4}}{a_{2}}\right) & C_{1}\left(a_{1}, a_{2}\right)=-\frac{3}{4 a_{2}}\left(\alpha^{2}+\mathrm{O}\left(\alpha^{4}\right)\right) \\
\mathrm{r}_{1}^{*}\left(a_{1}, a_{2}\right)=-\frac{3}{16 a_{2}}\left(\alpha^{2}+\mathrm{O}\left(\alpha^{4}\right)\right) & \mathrm{s}_{1}\left(a_{1}, a_{2}\right)=\frac{3}{a_{2}}\left(\alpha^{2}+\mathrm{O}\left(\alpha^{4}\right)\right) \\
\mathrm{s}_{1}\left(a_{2}, a_{1}\right)=\frac{9}{8 a_{2}}\left(\alpha^{2}+\mathrm{O}\left(\alpha^{4}\right)\right) & \mathrm{s}_{1}^{*}\left(a_{1}, a_{2}\right)=-\frac{3}{4 a_{2}}\left(\alpha^{2}+\mathrm{O}\left(\alpha^{4}\right)\right) \\
\mathrm{s}_{1}^{*}\left(a_{2}, a_{1}\right)=\frac{9}{8 a_{2}}\left(\alpha^{2}+\mathrm{O}\left(\alpha^{4}\right)\right) & \mathrm{s}_{2}\left(a_{1}, a_{2}\right), \mathrm{s}_{2}^{*}\left(a_{1}, a_{2}\right)=\mathrm{O}\left(\frac{\alpha^{3}}{a_{2}}\right)
\end{array}
$$

Moreover, by the definition of $d$ in $(71), d=\mathrm{O}(\alpha \sqrt{t})$, with $t:=\frac{\Lambda_{2}}{\Lambda_{1}}$. We thus obtain

$$
\breve{\mathrm{T}}=m_{1} m_{2} \frac{\alpha^{2}}{a_{2} \Lambda_{2}^{2}}\left(\begin{array}{ccc}
\frac{3}{4} t^{2} & -\frac{9}{4} t & 3 t^{2}-\frac{9}{4} t \\
-\frac{9}{4} t & -3 & \frac{9}{4} t+3 \\
3 t^{2}-\frac{9}{4} t & \frac{9}{4} t+3 & -\frac{3}{4}(1+t)^{2}-\frac{3}{4} t
\end{array}\right)\left(1+\mathrm{O}\left(\alpha^{2}\right)\right)
$$

b. Computation of $\hat{\mathrm{T}}\left(\Lambda_{1}, \Lambda_{2}\right)$ Using (73) and (68), we obtain

$$
\hat{\mathrm{T}}\left(\Lambda_{1}, \Lambda_{2}\right)=-\frac{3}{4} m_{1} m_{2} \frac{\alpha^{2}}{a_{2} \Lambda_{2}^{2}}\left(\begin{array}{ll}
1-3 t+8 t^{2} & 5+6 t+5 t^{2} \\
5+6 t+5 t^{2} & 13+9 t+t^{2}
\end{array}\right)\left(1+\mathrm{O}\left(\alpha^{2}\right)\right) .
$$

c. Computation of $\dot{\mathrm{T}}\left(\Lambda_{1}, \Lambda_{2} ; \mathrm{G}\right)$ and check of its non-singularity The matrix $\dot{\mathrm{T}}\left(\Lambda_{1}, \Lambda_{2} ; \mathrm{G}\right)$ defined in (67) is given by

$$
\dot{\mathrm{T}}\left(\Lambda_{1}, \Lambda_{2} ; \mathrm{G}\right)=-\frac{3}{4} m_{1} m_{2} \frac{\alpha^{2}}{a_{2} \Lambda_{2}^{2}}\left(\begin{array}{cc}
1+3 t+8 t^{2} & -5+6 t-5 t^{2} \\
-5+6 t-5 t^{2} & 13-9 t+t^{2}
\end{array}\right)\left(1+\mathrm{O}\left(\alpha^{2}\right)\right)+\mathrm{O}\left(\varepsilon_{2}^{2}\right) .
$$

It may vanish at most on a finite number of sub-manifolds of $\dot{\mathcal{L}}_{\mathrm{G}}$ :

$$
\operatorname{det} \dot{\mathrm{T}}\left(\Lambda_{1}, \Lambda_{2} ; \mathrm{G}\right)=-\frac{9}{16}\left(m_{1} m_{2} \frac{\alpha^{2}}{a_{2} \Lambda_{2}^{2}}\right)^{2}\left(12-90 t+8 t^{2}+9 t^{3}+17 t^{4}\right)\left(1+\mathrm{O}\left(\alpha^{2}\right)\right)+\mathrm{O}\left(\varepsilon_{2}^{2}\right) .
$$

\section{B. Existence of quasi-periodic motions}

Let us now take, as mentioned, $\alpha_{+}<\alpha_{s}$, where $\alpha_{s}$ is as in (44). Let $\dot{\mathrm{H}}$ be as in (65). We aim to apply the following result to $\dot{\mathrm{H}}$. Note that assumption $\left(\mathrm{A}_{1}\right)$ is trivially satisfied by $\mathrm{h}_{\mathrm{k}}$, while $\left(\mathrm{A}_{2}\right)$ and $\left(\mathrm{A}_{3}\right)$ have been discussed in Sections III A 5 and III A 6 .

Theorem III.1 (V.I.Arnold, Refs. ${ }^{3,7}$ ) Let $\mathcal{P}_{\varepsilon}:=V \times \mathbf{T}^{n_{1}} \times B_{\varepsilon}^{2 n_{2}}$, where $V$ is an open, bounded, connected set of $\mathbf{R}^{n_{1}}$ and $B_{\varepsilon}^{2 n_{2}}$ is a $2 n_{2}$-dimensional ball of radius $\varepsilon$ centered at 
the origin. Let $\varepsilon_{0}>0$ and let $H(I, \varphi, p, q ; \mu)=H_{0}(I)+\mu P(I, \varphi, p, q ; \mu)$ be a real-analytic Hamiltonian on $\mathcal{P}_{\varepsilon_{0}}$, endowed with the standard symplectic form $d I \wedge d \varphi+d p \wedge d q$. Assume that $H$ verifies the following non-degeneracy assumptions:

$\left(\mathrm{A}_{1}\right) I \in V \rightarrow \partial_{I} H_{0}$ is a diffeomorphism;

$\left(\mathrm{A}_{2}\right) P_{\mathrm{av}}(p, q ; I)=P_{0}(I)+\sum_{i=1}^{n_{2}} \Omega_{i}(I) r_{i}+\frac{1}{2} \sum_{i, j=1}^{n_{2}} \beta_{i j}(I) r_{i} r_{j}+\mathrm{O}_{5}(p, q ; I)$, with $r_{i}:=\frac{p_{i}^{2}+q_{i}^{2}}{2}$;

$\left(\mathrm{A}_{3}\right)|\operatorname{det} \beta(I)| \geq$ const $>0$ for all $I \in V$.

Then, there exist positive numbers $\varepsilon_{*}<\varepsilon_{0}, C_{*}$ and $c_{*}$ such that, for

$$
0<\varepsilon<\varepsilon_{*}, \quad 0<\mu<\frac{\varepsilon^{6}}{\left(\log \varepsilon^{-1}\right)^{c_{*}}},
$$

one can find a set $\mathcal{K} \subset \mathcal{P}_{\varepsilon}$ formed by the union of $H$-invariant $n$-dimensional Lagrangian tori, on which the $H$-motion is analytically conjugated to linear Diophantine quasi-periodic motions with frequencies $\left(\omega_{1}, \omega_{2}\right) \in \mathbf{R}^{n_{1}} \times \mathbf{R}^{n_{2}}$ with $\omega_{1}=O(1)$ and $\omega_{2}=O(\mu)$. The set $\mathcal{K}$ has positive Liouville-Lebesgue measure and satisfies

$$
\text { meas } \mathcal{P}_{\varepsilon}>\text { meas } \mathcal{K}>\left(1-C_{*} \sqrt{\varepsilon}\right) \text { meas } \mathcal{P}_{\varepsilon}
$$

We now aim to apply Theorem III.1 to the Hamiltonian $\dot{\mathrm{H}}_{\mathrm{G}}$ in (65), for any fixed $\mathrm{G} \in \mathbf{R}_{+}$. So, if $\dot{\varepsilon}_{1}$ as in Proposition III.4, $\varepsilon \in\left(0, \dot{\varepsilon}_{1}\right) ; \dot{\mathcal{L}}_{s, \mathrm{G}}$ is as in $(69)$ and $\dot{\mathcal{M}}_{\mathrm{G}, \varepsilon}:=\dot{\mathcal{L}}_{s, \mathrm{G}} \times \mathbf{T}^{2} \times B_{\varepsilon}^{4}$, we obtain the following

Theorem III.2 Let $\varepsilon$ and $\mu$ verify (74). Then the set $\dot{\mathcal{M}}_{\mathrm{G}, \varepsilon}$ contains a positive measure set $\dot{\mathcal{K}}_{\mathrm{G}}$ which is $\dot{\mathrm{H}}_{\mathrm{G}}$ invariant and is formed by the union of 4-dimensional Lagrangian, realanalytic tori on which the $\dot{\mathrm{H}}_{\mathrm{G}}$ motions are analytically conjugated to linear quasi-periodic motions. Furthermore, $\dot{\mathcal{K}}_{\mathrm{G}}$ satisfies the bound in $(75)$, with $\mathcal{P}_{\varepsilon}$ replaced by $\dot{\mathcal{M}}_{\mathrm{G}, \varepsilon}$.

\section{THE REDUCTION OF PERIHELIA}

This section contains a new coordinate system, which, just like the jrd coordinates in (11) is of the form "action-angle" and reduces completely the number of degrees of freedom, and, in addition, enjoys nice and useful parity properties. It is available for any number of planets (Ref. ${ }^{33}$ ), but we discuss it here only for two. 
We call Perihelia reduction for the three-body problem the set of coordinates

$$
\mathrm{p}:=\left(\Lambda_{1}, \Lambda_{2}, \mathrm{G}_{2}, \Theta, \mathrm{G}, \mathrm{Z}, \ell_{1}, \ell_{2}, \mathrm{~g}_{2}, \vartheta, \mathrm{g}, \zeta\right)
$$

defined as follows.

Consider the sets of $(x, y)$ such that the Keplerian motions $t \rightarrow\left(x^{(j)}(t), y^{(j)}(t)\right)$ generated by the Hamiltonians (5) starting from $\left(x^{(j)}, y^{(j)}\right)$ are ellipses with non-vanishing eccentricities. Let $a_{i}$ denote their semi-major axes; $\mathrm{C}^{(i)}:=x^{(i)} \times y^{(i)}$ the angular momenta; $\mathrm{P}^{(i)}$, with $\left|\mathrm{P}^{(i)}\right|=1$, the direction of the $i^{\text {th }}$ perihelion. Define the " $\mathrm{p}-$ nodes"

$$
\nu_{1}:=k^{(3)} \times \mathrm{C}, \quad \mathrm{n}_{1}:=\mathrm{C} \times \mathrm{P}^{(1)}, \quad \nu_{2}:=\mathrm{P}^{(1)} \times \mathrm{C}^{(2)}, \quad \mathrm{n}_{2}=\mathrm{C}^{(2)} \times \mathrm{P}^{(2)}
$$

and assume that they do not vanish. If, as in the definition of jrd, for any three vectors $u, v$, $w \in \mathbf{R}^{3}$, with $u, v \perp w$, let $\alpha_{w}(u, v)$ denote the oriented angle formed from $u$ to $v$, relative to the positive direction established by $w$, then define

$$
\left\{\begin{array} { l } 
{ \mathrm { Z } : = \mathrm { C } \cdot k ^ { ( 3 ) } } \\
{ \Theta : = \mathrm { C } \cdot \mathrm { P } ^ { ( 1 ) } = \mathrm { C } ^ { ( 2 ) } \cdot \mathrm { P } ^ { ( 1 ) } } \\
{ \mathrm { G } : = \| \mathrm { C } \| } \\
{ \mathrm { G } _ { 2 } : = \| \mathrm { C } ^ { ( 2 ) } \| } \\
{ \Lambda _ { j } : = \mathrm { M } _ { j } \sqrt { \mathrm { m } _ { j } a _ { j } } }
\end{array} \quad \left\{\begin{array}{l}
\zeta:=\alpha_{k^{(3)}}\left(k^{(1)}, \nu_{1}\right) \\
\vartheta:=\alpha_{\mathrm{P}^{(1)}}\left(\mathrm{n}_{1}, \nu_{2}\right) \\
\mathrm{g}:=\alpha_{\mathrm{C}}\left(\nu_{1}, \mathrm{n}_{1}\right) \\
\mathrm{g}_{2}:=\alpha_{\mathrm{C}^{(2)}}\left(\nu_{2}, \mathrm{n}_{2}\right) \\
\ell_{j}:=\text { mean anomaly of } x^{(j)}
\end{array}\right.\right.
$$

with $j=1,2$. Note that such definitions, together with assumptions of non-vanishing of the nodes (76), imply that

$$
\min \left\{G_{2}, G\right\}>0, \quad-G<Z<G, \quad-\min \left\{G_{2}, G\right\}<\Theta<\min \left\{G_{2}, G\right\} .
$$

In Section $\mathrm{V}$ we shall show that the map

$$
\phi_{\mathrm{p}}: \quad \mathrm{p}:=(\mathrm{Z}, \Theta, \chi, \Lambda, \zeta, \vartheta, \kappa, \ell) \in \mathbf{R}^{6} \times \mathbf{T}^{6} \rightarrow \mathcal{C}_{\text {art }}\left(y_{\mathrm{p}}^{(1)}, y_{\mathrm{p}}^{(2)}, x_{\mathrm{p}}^{(1)}, x_{\mathrm{p}}^{(2)}\right) \in \mathbf{R}^{12}
$$

preserves that standard 2-form:

$$
d \mathrm{Z} \wedge d \zeta+d \mathrm{G} \wedge d \mathrm{~g}+d \Theta \wedge d \vartheta+d \mathrm{G}_{2} \wedge d \mathrm{~g}_{2}+d \Lambda_{1} \wedge d \ell_{1}+d \Lambda_{2} \wedge d \ell_{2}=\sum_{j=1}^{2} \sum_{i=1}^{3} d y_{\mathrm{p}, i}^{(j)} \wedge d y_{\mathrm{p}, i}^{(j)}
$$

\section{Analytical expression of $\phi_{\mathrm{p}}$}

The explicit form of $\phi_{\mathrm{p}}$ is as follows. Let $\mathrm{e}_{2}, \zeta_{2}, i, R_{1}, R_{3}$ be as in (7), (A1), (A2), (A3), (A4). Let $\iota_{1}, \iota_{2}$ be the convex angles

$$
\cos \iota_{1}=\frac{\Theta}{\mathrm{G}}, \quad \cos \iota_{2}=\frac{\Theta}{\mathrm{G}_{2}} .
$$


By (78), one has $\iota_{1}, \iota_{2} \in(0, \pi)$. Then the expression of $\mathrm{C}, \mathrm{C}^{(2)}, \mathrm{P}^{(1)}, \mathrm{P}^{(2)}$ in terms of $\mathrm{p}$ are

$$
\begin{aligned}
& \mathrm{C}=\mathrm{G}_{3}(\zeta) R_{1}(i) e_{3}, \quad \mathrm{P}^{(1)}=R_{3}(\zeta) R_{1}(i) R_{3}(\mathrm{~g}) R_{1}\left(\iota_{1}\right) e_{3} \\
& \mathrm{C}^{(2)}=\mathrm{G}_{2} R_{3}(\zeta) R_{1}(i) R_{3}(\mathrm{~g}) R_{1}\left(\iota_{1}\right) R_{3}(\vartheta) R_{1}\left(\iota_{2}\right) e_{3} \\
& \mathrm{P}^{(2)}=R_{3}(\zeta) R_{1}(i) R_{3}(\mathrm{~g}) R_{1}\left(\iota_{1}\right) R_{3}(\vartheta) R_{1}\left(\iota_{2}\right) R_{3}\left(\mathrm{~g}_{2}-\pi / 2\right) e_{1}
\end{aligned}
$$

while $\mathrm{C}^{(1)}$ is found via

$$
\mathrm{C}^{(1)}=\mathrm{C}-\mathrm{C}^{(2)}=R_{3}(\zeta) R_{1}(i)\left(\mathrm{G} \mathbf{1}-\mathrm{G}_{2} R_{3}(\mathrm{~g}) R_{1}\left(\iota_{1}\right) R_{3}(\vartheta) R_{1}\left(\iota_{2}\right)\right) e_{3}
$$

In particular, $\mathrm{G}_{1}:=\left\|\mathrm{C}^{(1)}\right\|$ is not an action coordinate, but has the expression

$$
\mathrm{G}_{1}=\sqrt{\mathrm{G}^{2}+\mathrm{G}_{2}^{2}-2 \Theta^{2}+2 \sqrt{\mathrm{G}^{2}-\Theta^{2}} \sqrt{\mathrm{G}_{2}^{2}-\Theta^{2}} \cos \vartheta} .
$$

Such expression allows to find $e_{1}$ via $(A 1)$ as

$$
e_{1}=\sqrt{1-\frac{G^{2}+G_{2}^{2}-2 \Theta^{2}+2 \sqrt{G^{2}-\Theta^{2}} \sqrt{G_{2}^{2}-\Theta^{2}} \cos \vartheta}{\Lambda_{1}^{2}}}
$$

Letting now

$$
\mathrm{Q}^{(1)}:=\frac{\mathrm{C}^{(1)}}{\mathrm{G}_{1}} \times \mathrm{P}^{(1)}, \quad \mathrm{Q}^{(2)}:=\frac{\mathrm{C}^{(2)}}{\mathrm{G}_{2}} \times \mathrm{P}^{(2)}
$$

then, $x_{\mathrm{p}}^{(j)}:=x^{(j)}, y_{\mathrm{p}}^{(j)}:=y^{(j)}$ are, classically, given by

$$
\left\{\begin{array}{l}
x^{(j)}=\frac{\Lambda_{j}^{2}}{\mathrm{M}_{j} \mathrm{~m}_{j}^{2}}\left(\left(\cos \zeta_{j}-\mathrm{e}_{j}\right) \mathrm{P}^{(j)}+\sqrt{1-\mathrm{e}_{j}^{2}} \sin \zeta_{j} \mathrm{Q}^{(j)}\right) \\
y^{(j)}=\frac{\mathrm{M}_{j} \mathrm{~m}_{j}^{2}}{\Lambda_{j}}\left(-\frac{\cos \zeta_{j}}{1-\mathrm{e}_{j} \cos \zeta_{j}} \mathrm{P}^{(j)}+\sqrt{1-\mathrm{e}_{j}^{2}} \frac{\cos \zeta_{j}}{1-\mathrm{e}_{j} \cos \zeta_{j}} \mathrm{Q}^{(j)}\right)
\end{array}\right.
$$

where $\zeta_{j}$ solves (A2). Observe that, while the expression of $\mathrm{Q}^{(2)}$ in $(82)$ is relatively simple:

$$
\mathrm{Q}^{(2)}=\frac{\mathrm{C}^{(2)}}{\mathrm{G}_{2}} \times \mathrm{P}^{(2)}=R_{3}(\zeta) R_{1}(i) R_{3}(\mathrm{~g}) R_{1}\left(\iota_{1}\right) R_{3}(\vartheta) R_{1}\left(\iota_{2}\right) R_{3}\left(\mathrm{~g}_{2}\right) e_{1}
$$

the one of $\mathrm{Q}^{(1)}$ is much more involved. Fortunately, it will be not needed in the paper.

\section{Singularities}

By definition, the singularities of $\phi_{\mathrm{p}}$ arise when

$$
\begin{aligned}
& Z=G \text { or } Z=G \text { or } \Theta=G \text { or } \Theta=G_{2} \text { or } G_{2}=\Lambda_{2} \\
& G_{1}=\sqrt{G^{2}+G_{2}^{2}-2 \Theta^{2}+2 \sqrt{G^{2}-\Theta^{2}} \sqrt{G_{2}^{2}-\Theta^{2}} \cos \vartheta}=\Lambda_{1} \text { or } G_{2}=0 \text { or } G=0 \\
& \text { or }\left(G_{2}=G \text { and } \vartheta=\pi\right) .
\end{aligned}
$$


Note that the last line corresponds to the vanishing of $\mathrm{G}_{1}$ in (81). These formulae show that, compared to $\phi_{\mathrm{jrd}}, \phi_{\mathrm{p}}$ has the advantage that there are not singularities for vanishing inclinations. As a counterpart, while the singularity for $\mathrm{G}_{2}=\Lambda_{2}$ can be eliminated switching to the regularized (planar) Poincaré coordinates

$$
\left(\Lambda_{2}, \ell_{2}, \mathrm{G}_{2}, \mathrm{~g}_{2}\right) \rightarrow\left(\Lambda_{2}, \mathrm{l}_{2}, u_{2}, v_{2}\right):=\left(\Lambda_{2}, \ell_{2}+\mathrm{g}_{2}, \sqrt{2\left(\Lambda_{2}-\mathrm{G}_{2}\right)} \cos \mathrm{g}_{2},-\sqrt{2\left(\Lambda_{2}-\mathrm{G}_{2}\right)} \sin \mathrm{g}_{2}\right)
$$

for the spatial problem, a regularization for the singularity for $G_{1}=\Lambda_{1}$ does not seem to be possible, due to the non-linear expression of $\mathrm{G}_{1}$ in (81).

\section{Symmetries and planar equilibria}

Let

$$
\mathrm{H}_{\mathrm{p}}:=\mathrm{H} \circ \phi_{\mathrm{p}}=\mathrm{h}_{\mathrm{k}}\left(\Lambda_{1}, \Lambda_{2}\right)+\mu f_{\mathrm{p}}\left(\Lambda_{1}, \Lambda_{2}, \mathrm{G}_{2}, \Theta ; \ell_{1}, \ell_{2}, \mathrm{~g}_{2}, \vartheta ; \mathrm{G}\right)
$$

the Hamiltonian $\mathrm{H}$ expressed in terms of p. As well as $\mathrm{H}_{\mathrm{jrd}}$ in (16), $\mathrm{H}_{\mathrm{p}}$ is independent of $\mathrm{G}$, $\mathrm{Z}$ and $\mathrm{g}$. However, as an advantage with respect to the former, the symmetries by reflections induce a useful parity property to $\mathrm{H}_{\mathrm{p}}$, which does not exist for $\mathrm{H}_{\mathrm{jrd}}$. Indeed, it turns out that the transformation

$$
\mathcal{R}_{2}^{-}: \quad\left(\Lambda_{1}, \Lambda_{2}, \mathrm{G}_{2}, \Theta, \mathrm{G}, \mathrm{Z}, \ell_{1}, \ell_{2}, \mathrm{~g}_{2}, \vartheta, \mathrm{g}, \zeta\right) \rightarrow\left(\Lambda_{1}, \Lambda_{2}, \mathrm{G}_{2},-\Theta, \mathrm{G},-\mathrm{Z}, \ell_{1}, \ell_{2}, \mathrm{~g}_{2},-\vartheta, \mathrm{g},-\zeta\right)
$$

corresponds, in the Cartesian coordinates in (2), to

$$
\mathcal{R}_{2}^{-}: \quad\left(y_{1}^{(j)}, y_{2}^{(j)}, y_{3}^{(j)}, x_{1}^{(j)}, x_{2}^{(j)}, x_{3}^{(j)}\right) \rightarrow\left(y_{1}^{(j)},-y_{2}^{(j)}, y_{3}^{(j)}, x_{1}^{(j)},-x_{2}^{(j)}, x_{3}^{(j)}\right) \quad j=1,2
$$

This can be easily verified using the formulae given in Section IV 1 . Then, the $f_{\mathrm{p}}$ enjoys the following parity property

$$
f_{\mathrm{p}}\left(\Lambda_{1}, \Lambda_{2}, \mathrm{G}_{2}, \Theta ; \ell_{1}, \ell_{2}, \mathrm{~g}_{2}, \vartheta ; \mathrm{G}\right)=f_{\mathrm{p}}\left(\Lambda_{1}, \Lambda_{2}, \mathrm{G}_{2},-\Theta ; \ell_{1}, \ell_{2}, \mathrm{~g}_{2},-\vartheta ; \mathrm{G}\right) \bmod 2 \pi
$$

This equalities implies that the three manifolds

$$
\begin{aligned}
& (\uparrow \uparrow):=\left\{p:(\Theta, \vartheta)=(0, \pi) \text { and } G>G_{2}\right\} ; \\
& (\downarrow \uparrow):=\left\{p:(\Theta, \vartheta)=(0, \pi) \text { and } G<G_{2}\right\} ; \\
& (\uparrow \downarrow):=\{p:(\Theta, \vartheta)=(0,0)\} .
\end{aligned}
$$

are invariant to $f_{\mathrm{p}}$. Such manifolds correspond to planar motions, with the directions of the left, right arrows denoting the direction of the angular momentum of the inner, outer planet, respectively. The manifold $(\uparrow \downarrow)$ corresponds to planar ORC motions, which the object of study of this paper. 


\section{A domain of regularity including ( $\downarrow)$}

In this section we establish a suitable domain which includes the invariant manifold ( $\downarrow$ ) where $\mathrm{H}_{\mathrm{p}}$ is regular.

We check below that the following domain is suited to the scope:

$$
\mathcal{D}(\mathrm{G}):=\left\{\left(\Lambda_{1}, \Lambda_{2}, \mathrm{G}_{2}, \Theta, \vartheta\right):\left(\Lambda_{1}, \Lambda_{2}, \mathrm{G}_{2}\right) \in \mathcal{A}(\mathrm{G}),(\Theta, \vartheta) \in \mathcal{B}\left(\mathrm{G}_{2}, \mathrm{G}\right)\right\} \times \mathbf{T}^{3}
$$

where, if $\mathcal{L}$ is as in (33),

$$
\begin{aligned}
\mathcal{A}(\mathrm{G}) & :=\left\{\left(\Lambda_{1}, \Lambda_{2}, \mathrm{G}_{2}\right):\left(\Lambda_{1}, \Lambda_{2}\right) \in \mathcal{L}(\mathrm{G}), \mathrm{G}_{2} \in \mathcal{G}\left(\Lambda_{1}, \Lambda_{2}, \mathrm{G}\right)\right\} \\
\mathcal{B}\left(\mathrm{G}_{2}, \mathrm{G}\right) & :=\left\{(\Theta, \vartheta):|\Theta|<\frac{1}{2} \min \left\{\mathrm{G}, \mathrm{G}_{2}\right\},|\vartheta|<\frac{\pi}{2}\right\}
\end{aligned}
$$

with

$$
\begin{aligned}
\mathcal{L}(\mathrm{G}) & :=\left\{\Lambda=\left(\Lambda_{1}, \Lambda_{2}\right): \Lambda \in \mathcal{L}, \quad \Lambda_{1}>\mathrm{G}+\frac{2}{c} \sqrt{\alpha_{+}} \Lambda_{2}\right\} \\
\mathcal{G}\left(\Lambda_{1}, \Lambda_{2}, \mathrm{G}\right) & :=\left(\mathrm{G}_{-}, \mathrm{G}_{+}\right), \quad \mathrm{G}_{-}:=\frac{2}{c} \sqrt{\alpha_{+}} \Lambda_{2} \quad \mathrm{G}_{+}:=\min \left\{\Lambda_{1}-\mathrm{G}, \Lambda_{2}\right\} .
\end{aligned}
$$

where $\mathcal{L}$ is as in (33), while $c$ is an arbitrarily fixed number in $(0,1)$. We need to establish two kinds of conditions.

a. Geometric conditions First of all, we need that the planets' eccentricities $e_{1}, e_{2}$ stay strictly confined in $(0,1)$. Then the following inequalities are to be satisfied:

$$
0<\left\|\mathrm{C}_{\mathrm{p}}^{(1)}\right\|<\Lambda_{1} \quad 0<\mathrm{G}_{2}<\Lambda_{2}
$$

with $\left\|\mathrm{C}_{\mathrm{p}}^{(1)}\right\|$ as in (81). As remarked above, $\left\|\mathrm{C}_{\mathrm{p}}^{(1)}\right\|$ may vanish only for

$$
\left(\mathrm{G}_{2}, \vartheta\right)=(\mathrm{G}, \pi)
$$

Since we deal with the equilibrium $(\uparrow \downarrow)$ (which holds for $(\Theta, \vartheta)=(0,0)$ ), the occurrence of this equality is automatically excluded, limiting the values of the coordinates $(\Theta, \vartheta)$ in the set $\mathcal{B}$ in (86) since in this case

$$
\left\|\mathrm{C}_{\mathrm{p}}^{(1)}\right\|^{2} \geq \frac{3}{4} \mathrm{G}^{2}
$$

Moreover, the two right inequalities in (87) are satisfied taking

$$
\mathrm{G}_{2}<\min \left\{\Lambda_{1}-\mathrm{G}, \Lambda_{2}\right\}=\mathrm{G}_{+}
$$

where we have used the triangular inequality $\left\|\mathrm{C}_{\mathrm{p}}^{(1)}\right\| \leq\left\|\mathrm{C}_{\mathrm{p}}\right\|+\left\|\mathrm{C}_{\mathrm{p}}^{(2)}\right\|=\mathrm{G}+\mathrm{G}_{2}$. 
b. Non-collision conditions We have to exclude possible encounters of the planets with the sun and each other. Collisions of the inner planet with the sun are excluded by (86). Indeed, using (88), with $\Lambda_{1}:=k_{+} \Lambda_{2}$,

$$
1-e_{1}^{2}=\frac{\left\|\mathrm{C}_{\mathrm{p}}^{(1)}\right\|^{2}}{\Lambda_{1}^{2}} \geq \frac{3}{4} \frac{\mathrm{G}^{2}}{\left(\Lambda_{1}\right)^{2}}
$$

whence the minimum distance of the inner planet with the sun $a_{1}\left(1-e_{1}\right)$ is positive. In order to avoid planetary collisions, it is typical to ensure the following inequality:

$$
a_{1}\left(1+e_{1}\right)<c^{2} a_{2}\left(1-e_{2}\right)
$$

with $0<c<1$. A sufficient condition for it is

$$
\mathrm{G}_{2} \geq \frac{2}{c} \sqrt{\alpha_{+}} \Lambda_{2}=\mathrm{G}_{-}
$$

Indeed, if this inequality is satisfied, one has

$$
a_{1}\left(1+e_{1}\right)<2 a_{1}<\frac{a_{2}}{2} \frac{\mathrm{G}_{2}^{2} c^{2}}{\Lambda_{2}^{2}}=\frac{a_{2}}{2}\left(1-e_{2}^{2}\right) c^{2}<a_{2}\left(1-e_{2}\right) c^{2} .
$$

\section{General properties of the secular problem}

We call Kepler map any canonical change of coordinates

$$
\mathrm{k}=\left(\Lambda_{1}, \Lambda_{2}, \ell_{1}, \ell_{2}, u, v\right) \in \mathcal{L} \times \mathbf{T}^{2} \times V \rightarrow\left(y_{\mathrm{k}}^{(1)}, y_{\mathrm{k}}^{(2)}, x_{\mathrm{k}}^{(1)}, x_{\mathrm{k}}^{(2)}\right) \in \mathbf{R}^{12}
$$

such that

$$
\frac{\left|y_{\mathrm{k}}^{(j)}\right|^{2}}{2 \mathrm{~m}_{j}}-\frac{\mathrm{m}_{j} \mathrm{M}_{j}}{\left|x_{\mathrm{k}}^{(j)}\right|}=\mathrm{h}_{\mathrm{k}}^{(j)}\left(\Lambda_{j}\right) \quad j=1,2,
$$

where $\mathcal{L} \subset \mathbf{R}^{2}, V \subset \mathbf{R}^{8}$ are open and connected sets, and $\mathrm{h}_{\mathrm{k}}^{(j)}$ is as in (6). Different Kepler maps are related by canonical changes

$$
\mathrm{k}=\left(\Lambda_{1}, \Lambda_{2}, \ell_{1}, \ell_{2}, u, v\right) \rightarrow \mathrm{k}^{\prime}=\left(\Lambda_{1}, \Lambda_{2}, \ell_{1}^{\prime}, \ell_{2}^{\prime}, u^{\prime}, v^{\prime}\right)
$$

which leave the $\Lambda_{i}$ 's unvaried. In terms of any Kepler map the Hamiltonian (2) takes the aspect

$$
\mathrm{H}_{\mathrm{k}}=\mathrm{h}_{\mathrm{k}}\left(\Lambda_{1}, \Lambda_{2}\right)+\mu f_{\mathrm{k}}\left(\Lambda_{1}, \Lambda_{2}, \ell_{1}, \ell_{2}, u, v\right)
$$

where 


$$
\mathrm{h}_{\mathrm{k}}\left(\Lambda_{1}, \Lambda_{2}\right)=-\frac{\mathrm{m}_{1}^{3} \mathrm{M}_{1}^{2}}{2 \Lambda_{1}^{2}}-\frac{\mathrm{m}_{2}^{3} \mathrm{M}_{2}^{2}}{2 \Lambda_{2}^{2}}, \quad f_{\mathrm{k}}=-\frac{m_{1} m_{2}}{\left|x_{\mathrm{k}}^{(1)}-x_{\mathrm{k}}^{(2)}\right|}+\frac{y_{\mathrm{k}}^{(1)} \cdot y_{\mathrm{k}}^{(2)}}{m_{0}} .
$$

If $\mathrm{k}$ is any Kepler map, the "secular problem" is the $\left(\ell_{1}, \ell_{2}\right)$-independent system

$$
\mathrm{H}^{\mathrm{av}}=\mathrm{h}_{\mathrm{k}}+\mu f_{\mathrm{k}}^{\mathrm{av}}
$$

with

$$
f_{\mathrm{k}}^{\mathrm{av}}:=\frac{1}{(2 \pi)^{2}} \int_{[0,2 \pi]^{2}} f_{\mathrm{k}} d \ell_{1} d \ell_{2} .
$$

Some properties are enjoyed by all such systems, and are listed below.

a. The indirect part in the perturbing function (90) does not contribute to $f_{\mathrm{k}}^{\text {av }}$. Indeed, for any Kepler map, the impulses $y_{\mathrm{k}}^{(j)}$ satisfy

$$
y_{\mathrm{k}}^{(j)}=\frac{\mathrm{m}_{j}^{2} \mathrm{M}_{j}}{\Lambda_{j}} \partial_{\ell_{j}} \widehat{x}_{\mathrm{k}}^{(j)}, \quad \text { with } \quad \widehat{x}_{\mathrm{k}}^{(j)}:=\frac{x_{\mathrm{k}}^{(j)}}{a_{j}} .
$$

b. Expanding the averaged Newtonian potential in terms of the semi-axes ratio

$$
f_{\mathrm{k}}^{\mathrm{av}}=-\frac{m_{1} m_{2}}{a_{2}}\left(f_{\mathrm{k}}^{\mathrm{av}(0)}+f_{\mathrm{k}}^{\mathrm{av}(1)}+\cdots\right)
$$

then

$$
f_{\mathrm{k}}^{\mathrm{av}(0)}=1 \quad f_{\mathrm{k}}^{\mathrm{av}(1)} \equiv 0 .
$$

Indeed, $f_{\mathrm{k}}^{\mathrm{av}(0)}$ is the averaged Keplerian potential, given by $\frac{1}{a_{2}}$, while $f_{\mathrm{k}}^{\mathrm{av}(1)}$ is the average of $x_{\mathrm{k}}^{(1)} \cdot \frac{x_{\mathrm{k}}^{(2)}}{\left\|x_{\mathrm{k}}^{(1)}\right\|^{3}}$, which vanishes, because the second term is proportional to $\partial_{\ell_{2}} y_{\mathrm{k}}^{(2)}$.

c. The term of order 2 in the expansion (93) admits $\mathrm{G}_{2}$ as a first integral. This circumstance has been pointed out in Ref. ${ }^{16}$. In particular, in all Kepler maps such that $\mathrm{G}_{2}$ is an action, $f_{\mathrm{k}}^{\mathrm{av}(2)}$ does not depend on its conjugate variable, and hence depends on one conjugated couple only. Therefore, the secular, truncated system

$$
\mathrm{h}_{\mathrm{eff}}=\mathrm{h}_{\mathrm{k}}-\mu \frac{\mathrm{m}_{1} m_{2}}{a_{2}}\left(1+f_{\mathrm{k}}^{\mathrm{av}(2)}\right)
$$

which is obtained from (91) by truncating terms of order $\mathrm{O}\left(\mu \alpha^{3}\right)$, is integrable and, in particular, one-dimensional. This property holds true, in particular, for jrd and p. In the case of jrd, $f_{\mathrm{jrd}}^{\text {av }}(2)$, depends on the couple $\left(\mathrm{G}_{1}, \gamma_{1}\right)$, whose bifurcation diagram has been studied, e.g., in Refs. ${ }^{14,16,24}$. In the case of $\mathrm{p}, f_{\mathrm{p}}^{\text {av }(2)}$, depends on the couple $(\Theta, \vartheta)$ and, due 
to the equilibria (85), the dynamics of (95) can be studied by convergent Birkhoff series. More in general, the following formula has been proved in Ref. ${ }^{33}$ (Appendix B):

$$
\begin{aligned}
f_{\mathrm{k}}^{\mathrm{av}(2)}=-\frac{\alpha^{2}}{8} & \frac{\Lambda_{2}^{3}}{\Lambda_{1}^{2}\left\|\mathrm{C}_{\mathrm{k}}^{(2)}\right\|^{5}}\left[5\left(3\left(\mathrm{P}_{\mathrm{k}}^{(1)} \cdot \mathrm{C}_{\mathrm{k}}^{(2)}\right)^{2}-\left\|\mathrm{C}_{\mathrm{k}}^{(2)}\right\|^{2}\right) \Lambda_{1}^{2}\right. \\
& \left.-3\left(4\left(\mathrm{P}_{\mathrm{k}}^{(1)} \cdot \mathrm{C}_{\mathrm{k}}^{(2)}\right)^{2}-\left\|\mathrm{C}_{\mathrm{k}}^{(2)}\right\|^{2}\right)\left\|\mathrm{C}_{\mathrm{k}}^{(1)}\right\|^{2}+3\left(\mathrm{C}_{\mathrm{k}}^{(1)} \times \mathrm{C}_{\mathrm{k}}^{(2)} \cdot \mathrm{P}_{\mathrm{k}}^{(1)}\right)^{2}\right] .
\end{aligned}
$$

\section{Instability features in the secular problem}

In the case of $\mathrm{k}=\mathrm{p}$, Equation (96) gives

$$
f_{\mathrm{p}}^{\mathrm{av}(2)}=\alpha^{2}\left(\mathrm{P}_{0}+\mathrm{P}_{1}\right)
$$

with

$$
\begin{aligned}
\mathrm{P}_{0}:=-\frac{1}{8} & \frac{\Lambda_{2}^{3}}{\Lambda_{1}^{2} \mathrm{G}_{2}^{3}}\left(-5 \Lambda_{1}^{2}+3\left(\mathrm{G}+\mathrm{G}_{2}\right)^{2}\right) \\
\mathrm{P}_{1}:=-\frac{1}{8} & \frac{\Lambda_{2}^{3}}{\Lambda_{1}^{2} \mathrm{G}_{2}^{5}}\left[15 \Lambda_{1}^{2} \Theta^{2}-3\left(4 \Theta^{2}-\mathrm{G}_{2}^{2}\right)\left(\mathrm{G}^{2}+\mathrm{G}_{2}^{2}-2 \Theta^{2}+2 \sqrt{\left(\mathrm{G}_{2}^{2}-\Theta^{2}\right)\left(\mathrm{G}^{2}-\Theta^{2}\right)} \cos \vartheta\right)\right. \\
& \left.\quad-3 \mathrm{G}_{2}^{2}\left(\mathrm{G}+\mathrm{G}_{2}\right)^{2}+3\left(\mathrm{G}_{2}^{2}-\Theta^{2}\right)\left(\mathrm{G}^{2}-\Theta^{2}\right) \sin ^{2} \vartheta\right] .
\end{aligned}
$$

Note that $f_{\mathrm{p}}^{\mathrm{av}(2)}$ has been split so that $\alpha^{2} \mathrm{P}_{0}=\left.f_{\mathrm{p}}^{\mathrm{av}(2)}\right|_{(\Theta, \vartheta)=(0,0)}$, whence $\mathrm{P}_{1}$ vanishes for $(\Theta, \vartheta)=(0,0)$. In the next proposition, we study the properties of $\mathrm{P}_{1}$.

Recall the definition of the sets $\mathcal{A}(\mathrm{G}), \mathcal{B}\left(\mathrm{G}_{2}, \mathrm{G}\right)$ in (86).

Proposition IV.1 For any fixed $\mathrm{G} \in \mathbf{R}_{+}$, there exist $\mathcal{A}_{\mathrm{u}}(\mathrm{G}) \subset \mathcal{A}(\mathrm{G})$ and a neighborhood $\mathcal{B}_{\mathrm{u}}$ of $(0,0)$, with $\mathcal{B}_{\mathrm{u}} \subset \mathcal{B}\left(\mathrm{G}_{2}, \mathrm{G}\right)$ for all $\mathrm{G}_{2} \in \mathcal{G}$, such that $\mathrm{H}_{\mathrm{p}}$ is real-analytic on

$$
\mathcal{D}_{\mathrm{u}}:=\mathcal{A}_{\mathrm{u}} \times \mathcal{B}_{\mathrm{u}} \times \mathrm{T}^{3}
$$

and $(\Theta, \vartheta)=(0,0)$ is a hyperbolic equilibrium point for $\mathrm{P}_{1}$. More precisely, there exist two functions $\omega, \Omega$ of $\Lambda_{1}, \Lambda_{2}, \mathrm{G}_{2}$ and the parameter $\mathrm{G}$, with $\omega>0$ such that, if

$$
p_{0}:=\frac{\Theta-\omega \vartheta}{\sqrt{2 \omega}}, \quad q_{0}:=\frac{\Theta+\omega \vartheta}{\sqrt{2 \omega}}
$$

one has

$$
\mathrm{P}_{1}=\Omega p_{0} q_{0}+\mathrm{O}\left(p_{0}, q_{0} ; \Lambda_{2}, \Lambda_{2}, \mathrm{G}_{2}, \mathrm{G}\right)^{4}
$$


We shall prove the proposition with

$$
\begin{aligned}
\Omega & :=-\frac{3}{4} \frac{\Lambda_{2}^{3}}{\Lambda_{1}^{2} \mathrm{G}_{2}^{4}} \sqrt{\left(5 \Lambda_{1}^{2} \mathrm{G}-\left(\mathrm{G}+\mathrm{G}_{2}\right)^{2}\left(4 \mathrm{G}+\mathrm{G}_{2}\right)\right)\left(\mathrm{G}_{2}-\mathrm{G}\right)} \\
\omega & :=\mathrm{GG}_{2} \sqrt{\frac{\mathrm{G}_{2}-\mathrm{G}}{5 \Lambda_{1}^{2} \mathrm{G}-\left(\mathrm{G}+\mathrm{G}_{2}\right)^{2}\left(4 \mathrm{G}+\mathrm{G}_{2}\right)}} \\
\mathcal{A}_{\mathrm{u}}(\mathrm{G}) & :=\left\{\left(\Lambda_{1}, \Lambda_{2}\right) \in \mathcal{L}_{\mathrm{u}}(\mathrm{G}), \quad \mathrm{G}_{2} \in \mathcal{G}_{\mathrm{u}}\left(\Lambda_{1}, \Lambda_{2}, \mathrm{G}\right)\right\} \\
\mathcal{B}_{\mathrm{u}}(\mathrm{G}) & :=\left\{(\Theta, \vartheta):|\Theta|<\frac{\mathrm{G}}{2},|\vartheta|<\frac{\pi}{2}\right\}
\end{aligned}
$$

where

$$
\begin{aligned}
& \mathcal{L}_{\mathrm{u}}(\mathrm{G}):=\left\{\Lambda=\left(\Lambda_{1}, \Lambda_{2}\right) \in \mathcal{L}:\right. 5 \Lambda_{1}^{2} \mathrm{G}-\left(\mathrm{G}+\frac{2}{c} \sqrt{\alpha_{+}} \Lambda_{1}\right)^{2}\left(4 \mathrm{G}+\frac{2}{c} \sqrt{\alpha_{+}} \Lambda_{1}\right)>0 \\
&\left.\Lambda_{2}>\mathrm{G}, \Lambda_{1}>\max \left\{\mathrm{G}+\frac{2}{c} \sqrt{\alpha_{+}} \Lambda_{2}, 2 \mathrm{G}\right\}\right\} \\
& \mathcal{G}_{\mathrm{u}}\left(\Lambda_{1}, \Lambda_{2}, \mathrm{G}\right):=\left(\overline{\mathrm{G}}_{-}, \overline{\mathrm{G}}_{+}\right)
\end{aligned}
$$

where $\mathcal{L}$ is as in (33) and, if $\mathrm{G}^{\star}\left(\Lambda_{1}, \mathrm{G}\right)$ is the unique positive root of the cubic polynomial $\mathrm{G}_{2} \rightarrow 5 \Lambda_{1}^{2} \mathrm{G}-\left(\mathrm{G}+\mathrm{G}_{2}\right)^{2}\left(4 \mathrm{G}+\mathrm{G}_{2}\right)$, then

$$
\overline{\mathrm{G}}_{-}:=\max \left\{\frac{2}{c} \sqrt{\alpha_{+}} \Lambda_{2}, \mathrm{G}\right\} \quad \overline{\mathrm{G}}_{+}:=\min \left\{\Lambda_{2}, \mathrm{G}^{\star}\right\} .
$$

Implicitly, we shall prove that

$$
\overline{\mathrm{G}}_{-}<\overline{\mathrm{G}}_{+}
$$

Proof. The expansion of $\mathrm{P}_{1}$ in $(98)$ around $(0,0)$ is

$$
\mathrm{P}_{1}=-\frac{1}{8} \frac{\Lambda_{2}^{3}}{\Lambda_{1}^{2} \mathrm{G}_{2}^{5}} \times\left[\frac{3}{\mathrm{G}} a\left(\Lambda_{1}, \mathrm{G}_{2} ; \mathrm{G}\right) \Theta^{2}+3 \mathrm{GG}_{2}^{2} b\left(\mathrm{G}_{2} ; \mathrm{G}\right) \vartheta^{2}+o_{2}(\Theta, \vartheta)\right]
$$

where

$$
a\left(\Lambda_{1}, \mathrm{G}_{2} ; \mathrm{G}\right):=5 \Lambda_{1}^{2} \mathrm{G}-\left(\mathrm{G}+\mathrm{G}_{2}\right)^{2}\left(4 \mathrm{G}+\mathrm{G}_{2}\right) \text { and } \quad b\left(\mathrm{G}_{2} ; \mathrm{G}\right):=\mathrm{G}-\mathrm{G}_{2} .
$$

Both $\mathrm{G}_{2} \rightarrow a\left(\Lambda_{1}, \mathrm{G}_{2} ; \mathrm{G}\right)$ and $\mathrm{G}_{2} \rightarrow b\left(\mathrm{G}_{2} ; \mathrm{G}\right)$, as functions of $\mathrm{G}_{2}$ decrease monotonically from a positive value (respectively, $\mathrm{G}\left(5 \Lambda_{1}^{2}-4 \mathrm{G}^{2}\right)$ and $\mathrm{G}$ ) to $-\infty$ as $\mathrm{G}_{2}$ increases from $\mathrm{G}_{2}=0$ to $\mathrm{G}_{2}=+\infty$. The function $a\left(\Lambda_{1}, \mathrm{G}_{2} ; \mathrm{G}\right)$ changes its sign for $\mathrm{G}_{2}$ equal to a suitable unique positive value $\mathrm{G}^{\star}\left(\Lambda_{1}, \mathrm{G}\right)$, while $b\left(\mathrm{G}_{2} ; \mathrm{G}\right)$ does it for $\mathrm{G}_{2}=\mathrm{G}$. We note that (i) inequality $\mathrm{G}<\min \left\{\mathrm{G}_{+}, \mathrm{G}^{\star}\right\}$ follows immediately from the assumptions (102) (in particular, the two last ones) and (ii), more generally, that $\mathrm{G}^{\star} \leq \mathrm{G}$ is equivalent to $\Lambda_{1} \leq 2 \mathrm{G}$. Since, for 
our purposes, we have to exclude $\mathrm{G}^{\star}=\mathrm{G}$ (otherwise, $a\left(\Lambda_{1}, \mathrm{G}_{2} ; \mathrm{G}\right.$ ) and $b\left(\mathrm{G}_{2} ; \mathrm{G}\right.$ ) would be simultaneously positive and simultaneously negative, and no hyperbolicity would be possible), we distinguish two cases.

(a) $\mathrm{G}>\frac{2}{c} \sqrt{\alpha_{+}} \Lambda_{2}$ and $\mathrm{G}+\frac{2}{c} \sqrt{\alpha_{+}} \Lambda_{2}<\Lambda_{1}<2 \mathrm{G}$. In this case $\mathrm{G}^{\star}<\mathrm{G}$. We show that no such $\mathcal{G}_{\mathrm{u}}$ can exist in this case. In fact, since $\mathrm{G}^{\star}<\mathrm{G}$, in order that the interval $\left(G^{\star}, G\right)$ and the set $\mathcal{G}$ have a non-empty intersection, one should have, necessarily, $\mathrm{G}_{+}=\sup \mathcal{G}>\mathrm{G}^{\star}$, hence, in particular, $\Lambda_{1}-\mathrm{G}>\mathrm{G}^{\star}$. Using the definition of $\mathrm{G}^{\star}$, this would imply $\Lambda_{1}>2 \mathrm{G}$, which is a contradiction.

(b) $\Lambda_{1}>\max \left\{2 \mathrm{G}, \mathrm{G}+\frac{2}{c} \sqrt{\alpha_{+}} \Lambda_{2}\right\}$. In this case $\mathrm{G}<\mathrm{G}^{\star}<\Lambda_{1}-\mathrm{G}$. In order that the interval $\left(\mathrm{G}, \mathrm{G}^{\star}\right)$ and the set $\mathcal{G}$ have a non-empty intersection, we need

$$
\mathrm{G}_{-}<\mathrm{G}^{\star} \quad \text { and } \quad \mathrm{G}_{+}>\mathrm{G}
$$

and such intersection will be given by the interval $\mathcal{G}_{\mathrm{u}}$ as in (102). Note that the definition of $\bar{G}_{+}$does not include $\Lambda_{1}-\mathrm{G}$ in the brackets because, as noted, $\mathrm{G}^{\star}<\Lambda_{1}-\mathrm{G}$. But (106) are equivalent to (102).

Remark IV.1 The "bifurcation" towards the hyperbolic behavior that Proposition IV.1 talks about does not appear in the case of the equilibria $(\uparrow \uparrow)$ and $(\downarrow \uparrow)$, which, in contrast, are always elliptic. Indeed, in such cases, one obtains an expansion analogous to (98), with the coefficients $a, b$ in (105) to be replaced by

$$
\widehat{a}=5 \Lambda_{1}^{2} \mathrm{G}-\left(\mathrm{G}-\mathrm{G}_{2}\right)^{2}\left(4 \mathrm{G}-\mathrm{G}_{2}\right), \quad \widehat{b}=\mathrm{G}+\mathrm{G}_{2} .
$$

Clearly, $\widehat{b}$ is positive for all $\mathrm{G}_{2}$ and $\widehat{a}$ is so for $\mathrm{G}_{2} \geq 4 \mathrm{G}$. On the other hand, when $\mathrm{G}_{2}<4 \mathrm{G}$, inequality $\left|\mathrm{G}-\mathrm{G}_{2}\right|<\Lambda_{1}$ implies

$$
\widehat{a} \geq \Lambda_{1}^{2}\left(\mathrm{G}+\mathrm{G}_{2}\right)>0 .
$$

Therefore, $\widehat{a}$ and $\widehat{b}$ have always the same (positive) sign. This circumstance has been worked out in Ref. ${ }^{33}$, in the more general situation with $N \geq 2$ planets, in order to infer the existence of quasi-periodic motions with maximal number of frequencies away from the constraint, we have talked about of in Sections II A-III B, of small eccentricities and inclinations. 


\section{Unperturbed hyperbolic motions}

Let us consider the Hamiltonian $\mathrm{H}_{\mathrm{p}}$ in (84), on the domain $\mathcal{D}_{\mathrm{u}}$ in (99). Standard averaging theory (e.g., Ref. ${ }^{37}$ (Normal Form Lemma)) allows to eliminate the dependence on $\ell_{1}, \ell_{2}$ at a higher order, conjugating, via a real-analytic, $\mu$-close to the identity, canonical transformation, $\mathrm{H}_{\mathrm{p}}$ to a new Hamiltonian, that we denote as

$$
\mathrm{H}_{\mathrm{p}}^{\prime}=\mathrm{h}_{\mathrm{k}}\left(\Lambda_{1}, \Lambda_{2}\right)+\mu f_{\mathrm{p}}^{\mathrm{av}}\left(\Lambda_{1}, \Lambda_{2}, \mathrm{G}_{2}, \Theta, \mathrm{g}_{2}, \vartheta ; \mathrm{G}\right)+\mu^{\sigma} f_{\mathrm{p}}^{\prime}\left(\Lambda_{1}, \Lambda_{2}, \mathrm{G}_{2}, \Theta, \ell_{1}, \ell_{2}, \mathrm{~g}_{2}, \vartheta ; \mathrm{G}\right)
$$

where $f_{\mathrm{p}}^{\text {av }}$ is as in (92), while $1<\sigma<2$. In view of (93), we can thus split $\mathrm{H}_{\mathrm{p}}^{\prime}$ as

$$
\mathrm{H}_{\mathrm{p}}^{\prime}=\mathrm{h}_{\mathrm{eff}}+f_{\mathrm{eff}}
$$

where $\mathrm{h}_{\mathrm{eff}}$ is as in (95), with $\mathrm{k}=\mathrm{p}$, namely,

$$
\mathrm{h}_{\mathrm{eff}}:=\mathrm{h}_{\mathrm{k}}\left(\Lambda_{1}, \Lambda_{2}\right)-\mu \frac{m_{1} m_{2}}{a_{2}}\left(1+\alpha^{2}\left(\mathrm{P}_{0}+\mathrm{P}_{1}\right)\right)
$$

while $f_{\mathrm{eff}}:=\mu \alpha^{3} \widehat{\mathrm{P}}+\mu^{\sigma} f_{\mathrm{p}}^{\prime}$ is a smaller remainder. The leading term, $\mathrm{h}_{\mathrm{eff}}$, possesses, by Proposition IV.1, a hyperbolic fixed point at $(\Theta, \vartheta)=(0,0)$ and hence a family of threedimensional tori with linear motions of $\left(\ell_{1}, \ell_{2}, \mathrm{~g}_{2}\right)$, having equation

$$
\mathcal{T}_{\Lambda_{1}^{\star}, \Lambda_{2}^{\star}, \mathrm{G}_{2}^{\star}}=\left\{\left(\Lambda_{1}, \Lambda_{2}, \mathrm{G}_{2}\right)=\left(\Lambda_{1}^{\star}, \Lambda_{2}^{\star}, \mathrm{G}_{2}^{\star}\right), \quad\left(\ell_{1}, \ell_{2}, \mathrm{~g}_{2}\right) \in \mathbf{T}^{3}, \quad(\Theta, \vartheta)=(0,0)\right\}
$$

parametrized by $\left(\Lambda_{1}^{\star}, \Lambda_{2}^{\star}, \mathrm{G}_{2}^{\star}\right) \in \mathcal{A}_{\mathrm{u}}(\mathrm{G})$. Together to such tori, the unperturbed system also possesses two four-dimensional manifolds, the whiskers,

$$
\begin{aligned}
& \mathcal{W}_{\Lambda_{1}^{\star}, \Lambda_{2}^{\star}, \mathrm{G}_{2}^{\star}, \varepsilon}^{\mathrm{s}, \mathrm{s}}=\left\{\left(\Lambda_{1}, \Lambda_{2}, \mathrm{G}_{2}\right)=\left(\Lambda_{1}^{\star}, \Lambda_{2}^{\star}, \mathrm{G}_{2}^{\star}\right),\left(\ell_{1}, \ell_{2}, \mathrm{~g}_{2}\right) \in \mathbf{T}^{3}, \quad q_{0}=0,\left|p_{0}\right|<\varepsilon\right\} \\
& \mathcal{W}_{\Lambda_{1}^{\star}, \Lambda_{2}^{\star}, \mathrm{G}_{2}^{\star}, \varepsilon}^{\mathrm{l}}=\left\{\left(\Lambda_{1}, \Lambda_{2}, \mathrm{G}_{2}\right)=\left(\Lambda_{1}^{\star}, \Lambda_{2}^{\star}, \mathrm{G}_{2}^{\star}\right),\left(\ell_{1}, \ell_{2}, \mathrm{~g}_{2}\right) \in \mathbf{T}^{3}, \quad p_{0}=0,\left|q_{0}\right|<\varepsilon\right\}
\end{aligned}
$$

(with $p_{0}, q_{0}$ as in (100)) including the tori $\mathcal{T}_{\Lambda_{1}^{\star}, \Lambda_{2}^{\star}, \mathrm{G}_{2}^{\star}}$ as subsets, with motions asymptotic to $\mathcal{T}_{\Lambda_{1}^{\star}, \Lambda_{2}^{\star}, \mathrm{G}_{2}^{\star}}$ in the future/past, respectively. It is a reasonable expectation (motivated by the well established KAM theory) that such structure (tori and related whiskers) is preserved in the whole system, at least for those $\mathcal{T}_{\Lambda_{1}^{\star}, \Lambda_{2}^{\star}, \mathrm{G}_{2}^{\star}}$ 's whose frequencies $\omega^{\star}:=$ $\left.\partial_{\left(\Lambda_{2}^{*}, \Lambda_{2}^{*}, \mathrm{G}_{2}^{*}\right)} \mathrm{h}_{\mathrm{eff}}\right|_{(\Theta, \vartheta)=(0,0)}$ are highly irrational (Diophantine).

\section{ON THE CANONICAL CHARACTER OF JACOBI-RADAU-DEPRIT AND P-COORDINATES}

In this section we discuss, in a unified way, the canonical character of the jrd coordinates in (13)-(11) and the p-coordinates in (77). In both cases, we reduce to the discussion to the 
case $N=2$, as needed in the paper. Both such sets of coordinates are defined for a general number of particles. The generalization of jrd can be deduced from the original Deprit's paper, Ref. ${ }^{11}$. See also Ref. ${ }^{34}$, for a direct, inductive approach. As for the generalization of p, one can look at Ref..$^{33}$.

The proof of the canonical character of jrd and p will be based on the Delaunay coordinates, that here we recall, and a simple lemma.

* Delaunay coordinates (see Ref. ${ }^{15}$ ), six for every body, here denoted as

$$
\mathrm{d}=\left(\Lambda_{j}, \mathrm{G}_{j}, \mathrm{H}_{j}, \ell_{j}, \overline{\mathrm{g}}_{j}, \mathrm{~h}_{j}\right) \quad j=1,2
$$

are defined as follows. The coordinates $\Lambda_{j}, \mathrm{G}_{j}, \ell_{j}$ are as in (11), while, if

$$
n_{j}:=k^{(3)} \times \mathrm{C}^{(j)}
$$

and $\alpha_{w}(u, v)$ as said in Section II A, then

$$
\overline{\mathrm{g}}_{j}=\alpha_{\mathrm{C}^{(j)}}\left(n_{j}, \mathrm{P}^{(j)}\right), \quad \mathrm{h}_{j}=\alpha_{k^{(3)}}\left(k^{(1)}, n_{j}\right), \quad \mathrm{H}_{j}=\mathrm{C}^{(j)} \cdot k^{(3)}=\mathrm{C}_{3}^{(j)}
$$

with $\left(k^{(1)}, k^{(2)}, k^{(3)}\right)$ a prefixed orthonormal frame in $\mathbf{R}^{3}$.

* Let $R_{e}(\alpha) \in \mathrm{SO}(3)$ be a rotation by an angle $\alpha$ around the unit vector $e$; let

$$
x(\alpha, \bar{x}):=R_{e}(\alpha) \bar{x} \forall \bar{x} \in \mathbf{R}^{3}, \quad \mathrm{C}(x, y):=x \times y \forall x, y \in \mathbf{R}^{3} .
$$

Then we have

Lemma V.1 Given $\bar{y} \in \mathbf{R}^{3}$ and varying $\bar{x}$ and $\alpha$,

$$
y \cdot d x=(\mathrm{C}(\bar{x}, \bar{y}) \cdot e) d \alpha+\bar{y} \cdot d \bar{x}=(\mathrm{C}(x, y) \cdot e) d \alpha+\bar{y} \cdot d \bar{x}
$$

Proof. Varying $\alpha$ and letting $\bar{x}, \bar{y}$ fixed,

$$
\begin{aligned}
y(\alpha, \bar{y}) \cdot d\left(R_{e}(\alpha) \bar{x}\right) & =\left(R_{e}(\alpha) \bar{y}\right) \cdot d\left(R_{e}(\alpha) \bar{x}\right)=\left(R_{e}(\alpha) \bar{y}\right) \cdot\left(e \times R_{e}(\alpha) \bar{x}\right) \\
& =\left(R_{e}(\alpha) \bar{x}\right) \times\left(R_{e}(\alpha) \bar{y}\right) \cdot e \\
& =C(\bar{x}, \bar{y}) \cdot e=C(x, y) \cdot e .
\end{aligned}
$$

Letting also $\bar{x}$ vary, one has the thesis. 
Now we proceed with proving the canonical character of jrd and p, by showing that they are canonically related to the coordinates $\mathrm{d}$ in (109). Since the couples $\left(\Lambda_{j}, \ell_{j}\right)$ are in common to d, jrd and p, namely,

$$
\mathrm{d}=\left(\Lambda_{1}, \Lambda_{2}, \ell_{1}, \ell_{2}, \widehat{\mathrm{d}}\right), \quad \operatorname{jrd}=\left(\Lambda_{1}, \Lambda_{2}, \ell_{1}, \ell_{2}, \widehat{\mathrm{jrd}}\right), \quad \mathrm{p}=\left(\Lambda_{1}, \Lambda_{2}, \ell_{1}, \ell_{2}, \widehat{\mathrm{p}}\right)
$$

with

$$
\begin{aligned}
& \widehat{\mathrm{d}}=\left(\mathrm{H}_{1}, \mathrm{H}_{2}, \mathrm{G}_{1}, \mathrm{G}_{2}, \mathrm{~h}_{1}, \mathrm{~h}_{2}, \overline{\mathrm{g}}_{1}, \overline{\mathrm{g}}_{2}\right), \widehat{\mathrm{jrd}}=\left(\mathrm{Z}, \mathrm{G}, \mathrm{G}_{1}, \mathrm{G}_{2}, \zeta, \gamma, \gamma_{1}, \gamma_{2}\right) \\
& \widehat{\mathrm{p}}=\left(\mathrm{Z}, \mathrm{G}, \Theta, \mathrm{G}_{2}, \zeta, \mathrm{g}, \vartheta, \mathrm{g}_{2}\right)
\end{aligned}
$$

and the changes

$$
\widehat{\mathrm{d}} \rightarrow \widehat{\mathrm{jrd}} \rightarrow \widehat{\mathrm{p}}
$$

do not depend on $\left(\Lambda_{j}, \ell_{j}\right)$, we just need to check that such changes in (112) are canonical. We shall prove that

Theorem V.1 The changes of coordinates in (112) preserve the standard 1-form:

$$
\sum_{i=1}^{2}\left(\mathrm{H}_{i} d \mathrm{~h}_{i}+\mathrm{G}_{i} d \bar{g}_{i}\right)=\mathrm{Z} d \zeta+\mathrm{G} d \gamma+\mathrm{G}_{1} d \gamma_{1}+\mathrm{G}_{2} d \gamma_{2}=\mathrm{Z} d \zeta+\mathrm{G} d \mathrm{~g}+\Theta d \vartheta+\mathrm{G}_{2} d \mathrm{~g}_{2} .
$$

We shall use many times the following definitions.

Definition V.1 If $n \perp n^{\prime} \in \mathbf{R}^{3}$, we denote as $\mathrm{F} \sim\left(n, \cdot, n^{\prime}\right)$ the orthonormal frame $\mathrm{F}=$ $\left(\frac{n}{|n|}, \frac{n^{\prime} \times n}{\left|n^{\prime} \times n\right|}, \frac{n^{\prime}}{\left|n^{\prime}\right|}\right)$.

Definition V.2 We denote as

$$
\mathrm{F} \rightarrow \mathrm{F}^{\prime}
$$

any couple $\left(\mathrm{F}, \mathrm{F}^{\prime}\right)$ of orthonormal frames, with $\mathrm{F}=(i, j, k), \mathrm{F}^{\prime}=\left(i^{\prime}, j^{\prime}, k^{\prime}\right)$, such that $i^{\prime} \|$ $\pm k \times k^{\prime}$.

Observe that, in such case, the transformation of coordinates which relates the coordinates $\mathrm{X}^{\prime}$ relatively to $\mathrm{F}^{\prime}$ to the coordinates $\mathrm{X}$ relatively to $\mathrm{F}$ is

$$
\mathrm{X}=R_{3}(\psi) R_{1}(\iota) \mathrm{X}^{\prime}
$$

where $R_{1}, R_{3}$ are as in (A4); $\iota$, the "mutual inclination between $\mathrm{F}$ and $\mathrm{F}^{\prime \prime}$, is the convex angle between $k$ and $k^{\prime}$, while $\psi$, called "longitude of the node of $\mathrm{F}^{\prime}$ with respect to $\mathrm{F}$, is defined by $\psi:=\alpha_{k}\left(i, i^{\prime}\right)$. 
Proof. Let $\mathrm{F}_{0}$ be a prefixed reference frame, and let $\mathrm{F}_{\mathrm{d}}^{(j)} \sim\left(n_{j}, \cdot, \mathrm{C}^{(j)}\right)$, with $n_{j}$ as in (110). Then we have

$$
\mathrm{F}_{0} \rightarrow \mathrm{F}_{\mathrm{d}}^{(j)}
$$

Let $\mathrm{P}^{(j)}$ be the coordinates of the $j^{\text {th }}$ perihelion with respect to $\mathrm{F}_{0}$, and denote as $\mathrm{Q}^{(j)}:=$ $\widehat{\mathrm{C}}^{(j)} \times \mathrm{P}^{(j)}$, with $\widehat{\mathrm{C}}^{(j)}=\frac{\mathrm{C}^{(j)}}{\left|\mathrm{C}^{(j)}\right|}$, so that $\left(\mathrm{P}^{(j)}, \mathrm{Q}^{(j)}, \widehat{\mathrm{C}}^{(j)}\right)$ is an orthonormal triple in $\mathbf{R}^{3}$. The coordinates of such vectors relatively to $\mathrm{F}_{\mathrm{d}}^{(j)}$, are

$$
\mathrm{P}_{\mathrm{d}}^{(j)}=R_{3}\left(\overline{\mathrm{g}}_{j}\right) e_{1} \quad \mathrm{Q}_{\mathrm{d}}^{(j)}=R_{3}\left(\overline{\mathrm{g}}_{j}\right) e_{2}
$$

Therefore, by (114), (115) and the definitions in (111), we have

$$
\mathrm{P}^{(j)}=R_{3}\left(\mathrm{~h}_{j}\right) R_{1}\left(\bar{i}_{j}\right) R_{3}\left(\overline{\mathrm{g}}_{j}\right) e_{1}, \quad \mathrm{Q}^{(j)}=R_{3}\left(\mathrm{~h}_{j}\right) R_{1}\left(\bar{i}_{j}\right) R_{3}\left(\overline{\mathrm{g}}_{j}\right) e_{2},
$$

where $\cos \bar{i}_{j}=\frac{\mathrm{H}_{j}}{\mathrm{G}_{j}}$. Then in view of Lemma V.1, we obtain (using $e_{1} \cdot R_{3}\left(\gamma_{j}\right)\left(e_{1} \times e_{2}\right)=$ $e_{1} \cdot e_{3}=0$ and $\left.\mathrm{P}^{(j)} \times \mathrm{Q}^{(j)}=\widehat{\mathrm{C}}^{(j)}\right)$

$$
\mathrm{Q}^{(j)} \cdot d \mathrm{P}^{(j)}=\widehat{\mathrm{C}}^{(j)} \cdot e_{3} d \mathrm{~h}_{j}+d \overline{\mathrm{g}}_{j} .
$$

Multiplying by $\mathrm{G}_{j}=\left|\mathrm{C}^{(j)}\right|$ and recognizing that $\mathrm{G}_{j} \widehat{\mathrm{C}}^{(j)} \cdot e_{3}=\mathrm{H}_{j}$, we then have

$$
\sum_{i=1}^{2}\left(\mathrm{H}_{i} d \mathrm{~h}_{i}+\mathrm{G}_{i} d \overline{\mathrm{g}}_{i}\right)=\sum_{j=1}^{2}\left|\mathrm{C}^{(j)}\right| \mathrm{Q}^{(j)} \cdot d \mathrm{P}^{(j)} .
$$

Now we compute the right hand side of this equation, using the jrd and $\mathrm{p}-$ coordinates. To this end, we need to express $\mathrm{P}^{(j)}$ and $\mathrm{Q}^{(j)}$ in terms of such two sets. To accomplish this, we observe that, in the sense of Definition V.2,

* In the case of jrd, we have the "tree" of changes of frames,

$$
\begin{aligned}
\mathrm{F}_{0} \rightarrow & \mathrm{F}_{*} \rightarrow \mathrm{F}_{\mathrm{jrd}}^{(1)} \\
& \downarrow \\
& \mathrm{F}_{\mathrm{jrd}}^{(2)}
\end{aligned}
$$

where $\mathrm{F}_{\mathrm{jrd}}^{(j)} \sim\left(\nu, \cdot \mathrm{C}^{(j)}\right)$, while $\mathrm{F}_{*} \sim\left(\nu_{1}, \cdot \mathrm{C}\right)$ is the invariable frame.

* In the case of $p$, we have the "chain"

$$
\mathrm{F}_{0} \rightarrow \mathrm{F}_{*} \rightarrow \mathrm{G}_{\mathrm{p}}^{(1)} \rightarrow \mathrm{F}_{\mathrm{p}}^{(2)} \rightarrow \mathrm{G}_{\mathrm{p}}^{(2)}
$$

where $\mathrm{F}_{0}, \mathrm{~F}_{*}$ are as in the previous item, while

$$
\mathrm{G}_{\mathrm{p}}^{(1)} \sim\left(\mathrm{n}_{1}, \cdot, \mathrm{P}^{(1)}\right), \quad \mathrm{F}_{\mathrm{p}}^{(2)} \sim\left(\nu_{2}, \cdot, \mathrm{C}^{(2)}\right), \quad \mathrm{G}_{\mathrm{p}}^{(2)} \sim\left(\mathrm{n}_{2}, \cdot, \mathrm{P}^{(2)}\right)
$$


Therefore,

* Recognizing (by the analysis of the triangle formed by $\mathrm{C}^{(1)}, \mathrm{C}^{(2)}$ and $\mathrm{C}=\mathrm{C}^{(1)}+\mathrm{C}^{(2)}$ ) that the inclinations $i, i_{1}, i_{2}$ between $\mathrm{F}_{0}$ and $\mathrm{F}_{*}, \mathrm{~F}_{*}$ and $\mathrm{F}_{\mathrm{jrd}}^{(1)}, \mathrm{F}_{*}$ and $\mathrm{F}_{\mathrm{jrd}}^{(2)}$ are given by (A3), while the longitudes of the nodes are, respectively, $\zeta, \gamma, \gamma+\pi$, we find the formulae

$$
\begin{aligned}
& \mathrm{P}^{(j)}=R_{3}(\zeta) R_{1}(i) R_{3}(\gamma) R_{1}\left(s_{j} i_{j}\right) R_{3}\left(\gamma_{j}\right) e_{1} \\
& \mathrm{Q}^{(j)}=R_{3}(\zeta) R_{1}(i) R_{3}(\gamma) R_{1}\left(s_{j} i_{j}\right) R_{3}\left(\gamma_{j}\right) e_{2}
\end{aligned}
$$

where $s_{1}=-s_{2}=1$. Here we have used $R_{3}(\pi) R_{1}(\alpha)=R_{1}(-\alpha) R_{3}(\pi)$ and the definitions of the angles $\gamma_{j}$ in (11). Observe, incidentally, that the formulae in (119) allow us to obtain the formulae in (A5), since, as known, $y^{(j)}, x^{(j)}$ are related to $a_{j}, \mathrm{e}_{j}, \mathrm{P}^{(j)}$ and $\mathrm{Q}^{(j)}$ via the classical relations in (83). with $a_{j}$ as in (7); $\mathrm{e}_{j}, \zeta_{j}$ as in (A1), (A2).

* The expressions of $\mathrm{P}^{(j)}, \mathrm{Q}^{(j)}$ in terms of the $\mathrm{p}$ coordinates have been given in $(80),(82)$.

We are now ready to compute the right hand side of (116), in terms of jrd and p. To this scope, we shall use Lemma V.1.

* Using the formulae in (119), iterate applications of Lemma V.1 and linear algebra, we obtain

$$
\mathrm{Q}^{(j)} \cdot d \mathrm{P}^{(j)}=\widehat{\mathrm{C}}^{(j)} \cdot e_{3} d \zeta+\widehat{\mathrm{C}}^{(j)} \cdot \nu d i+\widehat{\mathrm{C}}^{(j)} \cdot k d \gamma+\mathrm{f}_{j} \cdot e_{1} d i_{j}+d \gamma_{j}
$$

with $\nu:=R_{3}(\zeta) e_{1}=(\cos \zeta, \sin \zeta, 0), k:=R_{3}(\zeta) R_{1}(\iota) e_{3}, \mathrm{f}_{j}:=R_{1}\left(s_{j} i_{j}\right) e_{3}$. Multiplying by $\mathrm{G}_{j}=\left|\mathrm{C}^{(j)}\right|$, summing over $j=1,2$ and recognizing that $k$ has the direction of $\mathrm{C}, \nu$ is orthogonal to $\mathrm{C}$ and $\mathrm{G}_{1} \mathrm{f}_{1}+\mathrm{G}_{2} \mathrm{f}_{2}=\mathrm{Ge}_{3}$, we immediately obtain, after some cancellation,

$$
\sum_{j=1}^{(2)}\left|\mathrm{C}^{(j)}\right| \mathrm{Q}^{(j)} \cdot d \mathrm{P}^{(j)}=\mathrm{Z} d \zeta+\mathrm{G} d \gamma+\mathrm{G}_{1} d \gamma_{1}+\mathrm{G}_{2} d \gamma_{2}
$$

* Using the formulae in (80), defining $\widehat{\mathrm{C}}_{1}^{(1)}, \widehat{\mathrm{C}}_{2}^{(1)}, \widehat{\mathrm{C}}_{1}^{(2)}, \widehat{\mathrm{C}}_{2}^{(2)}, \widehat{\mathrm{C}}_{3}^{(2)}$ via

$$
\begin{aligned}
\widehat{\mathrm{C}}^{(1)} & =R_{3}(\zeta) R_{1}(i) \widehat{\mathrm{C}}_{1}^{(1)}=R_{3}(\zeta) R_{1}(i) R_{3}(\mathrm{~g}) R_{1}\left(i_{1}\right) \widehat{\mathrm{C}}_{2}^{(1)} \\
\widehat{\mathrm{C}}^{(2)} & =R_{3}(\zeta) R_{1}(i) \widehat{\mathrm{C}}_{1}^{(2)}=R_{3}(\zeta) R_{1}(i) R_{3}(\mathrm{~g}) R_{1}\left(i_{1}\right) \widehat{\mathrm{C}}_{2}^{(2)} \\
& =R_{3}(\zeta) R_{1}(i) R_{3}(\mathrm{~g}) R_{1}\left(i_{1}\right) R_{3}(\vartheta) R_{1}\left(i_{2}\right) \widehat{\mathrm{C}}_{3}^{(2)}
\end{aligned}
$$


and applying iteratively Lemma V.1, we obtain

$$
\begin{aligned}
\mathrm{Q}^{(1)} \cdot d \mathrm{P}^{(1)} & =\widehat{\mathrm{C}}^{(1)} \cdot e_{3} d \zeta+\widehat{\mathrm{C}}_{1}^{(1)} \cdot e_{1} d i+\widehat{\mathrm{C}}_{1}^{(1)} \cdot e_{3} d \mathrm{~g}+\widehat{\mathrm{C}}_{2}^{(1)} \cdot e_{1} d i_{1} \\
\mathrm{Q}^{(2)} \cdot d \mathrm{P}^{(2)} & =\widehat{\mathrm{C}}^{(2)} \cdot e_{3} d \zeta+\widehat{\mathrm{C}}_{1}^{(2)} \cdot e_{1} d i+\widehat{\mathrm{C}}_{1}^{(2)} \cdot e_{3} d \mathrm{~g}+\widehat{\mathrm{C}}_{2}^{(2)} \cdot e_{1} d i_{1} \\
& +\widehat{\mathrm{C}}_{2}^{(2)} \cdot e_{3} d \vartheta+\widehat{\mathrm{C}}_{3}^{(2)} \cdot e_{1} d i_{2}+\widehat{\mathrm{C}}_{3}^{(2)} \cdot e_{3} d \mathrm{~g}_{2}
\end{aligned}
$$

We multiply, as above, the first equation by $\left|\mathrm{C}^{(1)}\right|$, the second by $\left|\mathrm{C}^{(2)}\right|$, and take the sum of the two. The sum of the first three respective terms gives, analogously to the previous item, $\mathrm{Zd} \zeta+\mathrm{G} d \mathrm{~g}$. As for the remaining terms, we recognize that $\widehat{\mathrm{C}}_{3}^{(2)}=e_{3}$ so that $\widehat{\mathrm{C}}_{3}^{(2)} \cdot e_{1}=0, \widehat{\mathrm{C}}_{3}^{(2)} \cdot e_{3}=1 ; \widehat{\mathrm{C}}_{2}^{(2)} \cdot e_{3}=\Theta ;\left|\mathrm{C}^{(1)}\right| \widehat{\mathrm{C}}_{2}^{(1)} \cdot e_{1}+\left|\mathrm{C}^{(2)}\right| \widehat{\mathrm{C}}_{2}^{(2)} \cdot e_{1}=\left(\mathrm{C}^{(1)}+\right.$ $\left.\mathrm{C}^{(2)}\right) \cdot R_{3}(\zeta) R_{1}(i) R_{3}(\mathrm{~g}) R_{1}\left(i_{1}\right) e_{1}=\mathrm{C} \cdot R_{3}(\zeta) R_{1}(i) R_{3}(\mathrm{~g}) R_{1}\left(i_{1}\right) e_{1}=\mathrm{Ge}_{3} \cdot R_{3}(\mathrm{~g}) e_{1}=0$.

We finally obtain

$$
\sum_{j=1}^{2}\left|\mathrm{C}^{(j)}\right| \mathrm{Q}^{(j)} \cdot d \mathrm{P}^{(j)}=\mathrm{Zd \zeta}+\mathrm{G} d \mathrm{~g}+\Theta d \vartheta+\mathrm{G}_{2} d \mathrm{~g}_{2}
$$

The collection of (116), (120) and (121) proves Theorem V.1.

\section{Appendix A}

\section{Proof of Proposition III.1 and other technicalities}

The starting point is the analytical expression of $\phi_{\mathrm{jrd}}$, as presented in Refs. ${ }^{9,34}$, that here we recall.

\section{a. Analytical expression of $\phi_{\mathrm{jrd}}$}

Let $\mathrm{e}_{j} \in(0,1)$ be the eccentricity of the Keplerian orbit, as known, related to $\Lambda_{j}, \mathrm{G}_{j}$ via

$$
\mathrm{e}_{j}=\sqrt{1-\frac{\mathrm{G}_{j}^{2}}{\Lambda_{j}^{2}}}
$$

$\zeta_{j}\left(\mathrm{e}_{j}, \ell_{j}\right)$ the eccentric anomaly, defined as the unique solution of Kepler equation

$$
\zeta_{j}-\mathrm{e}_{j} \sin \zeta_{j}=\ell_{j} \quad j=1,2
$$

and let $i, i_{1}, i_{2}$ be defined via

$$
i=\cos ^{-1} \frac{\mathrm{Z}}{\mathrm{G}}, \quad i_{1}=\cos ^{-1} \frac{\mathrm{G}_{1}^{2}+\mathrm{G}^{2}-\mathrm{G}_{2}^{2}}{2 \mathrm{GG}_{1}}, \quad i_{2}=\cos ^{-1} \frac{\mathrm{G}_{2}^{2}+\mathrm{G}^{2}-\mathrm{G}_{1}^{2}}{2 \mathrm{GG}_{2}} .
$$


By $(12), i, i_{1}$ and $i_{2}$ take values on $(0, \pi)$. Let, finally, $R_{1}, R_{3}$ denote the matrices

$$
R_{1}(\alpha):=\left(\begin{array}{ccc}
1 & 0 & 0 \\
0 & \cos \alpha & -\sin \alpha \\
0 & \sin \alpha & \cos \alpha
\end{array}\right) \quad R_{3}(\alpha):=\left(\begin{array}{ccc}
\cos \alpha & -\sin \alpha & 0 \\
\sin \alpha & \cos \alpha & 0 \\
0 & 0 & 1
\end{array}\right) .
$$

The map $\phi_{\mathrm{jrd}}$ in (14) is defined as

$$
\left\{\begin{array}{l}
x_{\mathrm{jrd}}^{(j)}=R_{3}(\zeta) R_{1}(i) R_{3}(\gamma) R_{1}\left(s_{j} i_{j}\right) R_{3}\left(\gamma_{j}\right) x_{\mathrm{orb}}^{(j)}\left(\Lambda_{j}, \mathrm{G}_{j}, \ell_{j}\right) \\
y_{\mathrm{jrd}}^{(j)}=R_{3}(\zeta) R_{1}(i) R_{3}(\gamma) R_{1}\left(s_{j} i_{j}\right) R_{3}\left(\gamma_{j}\right) y_{\mathrm{orb}}^{(j)}\left(\Lambda_{j}, \mathrm{G}_{j}, \ell_{j}\right)
\end{array}\right.
$$

with $s_{1}=-s_{2}=1$ and

$$
\left\{\begin{array}{c}
x_{\mathrm{orb}}^{(j)}=\frac{\Lambda_{j}^{2}}{\mathrm{~m}_{j}^{2} \mathrm{M}_{j}}\left(\begin{array}{c}
\cos \zeta_{j}-\mathrm{e}_{j} \\
\sqrt{1-\mathrm{e}_{j}^{2}} \sin \zeta_{j} \\
0
\end{array}\right) \\
y_{\mathrm{orb}}^{(j)}=\frac{\mathrm{m}_{j}^{2} \mathrm{M}_{j}}{\Lambda_{j}} \frac{1}{1-\mathrm{e}_{j} \cos \zeta_{j}}\left(\begin{array}{c}
-\sin \zeta_{j} \\
\sqrt{1-\mathrm{e}_{j}^{2}} \cos \zeta_{j} \\
0
\end{array}\right)
\end{array}\right.
$$

The (A5) simply describe the successive rotations necessary to transform the coordinates of $x^{(j)}, y^{(j)}$ relatively to the "orbital frame" in (A6) into the coordinates relatively to the prefixed frame. Roughly, the choice $s_{1}=-s_{2}=1$ reflects the classical "opposition of the nodes" in the frame where the total angular momentum is vertical, i.e., the identity $\mathrm{C} \times \mathrm{C}^{(1)}+\mathrm{C} \times \mathrm{C}^{(2)}=\mathrm{C} \times \mathrm{C} \equiv 0$.

\section{b. Analytical expression of $\phi_{\mathrm{rps}}^{\mathrm{C}}$}

The formulae of the $\phi_{\mathrm{rps}}^{\mathbf{C}}$ map may be recovered rewriting (A5) in the form

$$
\left\{\begin{array}{l}
x_{\mathrm{jrd}}^{(j)}=\widetilde{\mathrm{R}}_{0} \widetilde{\mathrm{R}}_{j} \widetilde{\mathrm{x}}_{\mathrm{pl}}^{(j)} \\
y_{\mathrm{jrd}}^{(j)}=\widetilde{\mathrm{R}}_{0} \widetilde{\mathrm{R}}_{j} \widetilde{\mathrm{y}}_{\mathrm{pl}}^{(j)}
\end{array}\right.
$$

where

$$
\begin{aligned}
& \widetilde{\mathrm{R}}_{0}:=\widetilde{R}_{313}(\zeta, i), \quad \widetilde{\mathrm{R}}_{j}:=\widetilde{R}_{313}\left(\zeta+\gamma, s_{j} i_{j}\right), \quad \widetilde{\mathrm{x}}_{\mathrm{pl}}^{(j)}:=R_{3}\left(\zeta+\gamma+\gamma_{j}\right) x_{\mathrm{orb}}^{(j)}\left(\Lambda_{j}, \mathrm{G}_{j}, \ell_{j}\right) \\
& \widetilde{\mathrm{y}}_{\mathrm{pl}}^{(j)}:=R_{3}\left(\zeta+\gamma+\gamma_{j}\right) y_{\mathrm{orb}}^{(j)}\left(\Lambda_{j}, \mathrm{G}_{j}, \ell_{j}\right)
\end{aligned}
$$


with $x_{\text {orb }}^{(j)}, y_{\text {orb }}^{(j)}$ as in $(\mathrm{A} 6)$,

$$
\begin{aligned}
\widetilde{R}_{313}(\alpha, \beta) & :=R_{3}(\alpha) R_{1}(\beta) R_{3}(-\alpha) \\
& =\left(\begin{array}{ccc}
1-\sin ^{2} \alpha(1-\cos \beta) & \sin \alpha \cos \alpha(1-\cos \beta) & \sin \alpha \sin \beta \\
\sin \alpha \cos \alpha(1-\cos \beta) & 1-\cos ^{2} \alpha(1-\cos \beta) & -\cos \alpha \sin \beta \\
-\sin \alpha \sin \beta & \cos \alpha \sin \beta & \cos \beta
\end{array}\right)
\end{aligned}
$$

Note that, in particular, the matrices $\widetilde{R}_{0}, \widetilde{R}_{1}, \widetilde{R}_{2}$ are given by

$$
\begin{gathered}
\widetilde{\mathrm{R}}_{0}=\left(\begin{array}{ccc}
1-\sin ^{2} \zeta(1-\cos i) & \sin \zeta \cos \zeta(1-\cos i) & \sin \zeta \sin i \\
\sin \zeta \cos \zeta(1-\cos i) & 1-\cos ^{2} \zeta(1-\cos i) & -\cos \zeta \sin i \\
-\sin \zeta \sin i & \cos \zeta \sin i & \cos i
\end{array}\right) \\
\widetilde{\mathrm{R}}_{j}=\left(\begin{array}{ccc}
1-\sin ^{2}(\zeta+\gamma)\left(1-\cos i_{j}\right) & \sin (\zeta+\gamma) \cos (\zeta+\gamma)\left(1-\cos i_{j}\right) & s_{j} \sin (\zeta+\gamma) \sin i_{j} \\
\sin (\zeta+\gamma) \cos (\zeta+\gamma)\left(1-\cos i_{j}\right) & 1-\cos ^{2}(\zeta+\gamma)\left(1-\cos i_{j}\right) & -s_{j} \cos (\zeta+\gamma) \sin i_{j} \\
-s_{j} \sin (\zeta+\gamma) \sin i_{j} & s_{j} \cos (\zeta+\gamma) \sin i_{j} & \cos i_{j}
\end{array}\right) .
\end{gathered}
$$

Now, defining

$$
\widetilde{\mathcal{R}}_{0}=\widetilde{\mathrm{R}}_{0} \circ \phi_{\mathrm{rps}}^{\mathrm{jrd}}, \quad \widetilde{\mathcal{R}}_{j}=\widetilde{\mathrm{R}}_{j} \circ \phi_{\mathrm{rps}}^{\mathrm{jrd}}, \quad \widetilde{y}_{\mathrm{pl}}^{(j)}=\widetilde{\mathrm{y}}_{\mathrm{pl}}^{(j)} \circ \phi_{\mathrm{rps}}^{\mathrm{jrd}}, \quad \widetilde{y}_{\mathrm{pl}}^{(j)}=\widetilde{\mathrm{y}}_{\mathrm{pl}}^{(j)} \circ \phi_{\mathrm{rps}}^{\mathrm{jr}}
$$

we have, from (A7),

$$
\left\{\begin{array}{l}
x_{\mathrm{rps}}^{(j)}=\widetilde{\mathcal{R}}_{0} \widetilde{\mathcal{R}}_{j} \widetilde{x}_{\mathrm{pl}}^{(j)} \\
y_{\mathrm{rps}}^{(j)}=\widetilde{\mathcal{R}}_{0} \widetilde{\mathcal{R}}_{j} \widetilde{y}_{\mathrm{pl}}^{(j)}
\end{array}\right.
$$

We remark that the explicit expressions of $\widetilde{\mathcal{R}}_{0}, \widetilde{\mathcal{R}}_{j}, \widetilde{x}_{\mathrm{pl}}^{(j)}$ and $\widetilde{y}_{\mathrm{pl}}^{(j)}$ will be obtained from $\widetilde{\mathrm{R}}_{0}$, $\widetilde{\mathrm{R}}_{j}, \widetilde{\mathrm{x}}_{\mathrm{pl}}^{(j)}$ and $\widetilde{\mathrm{y}}_{\mathrm{pl}}^{(j)}$ replacing the appropriate arguments as

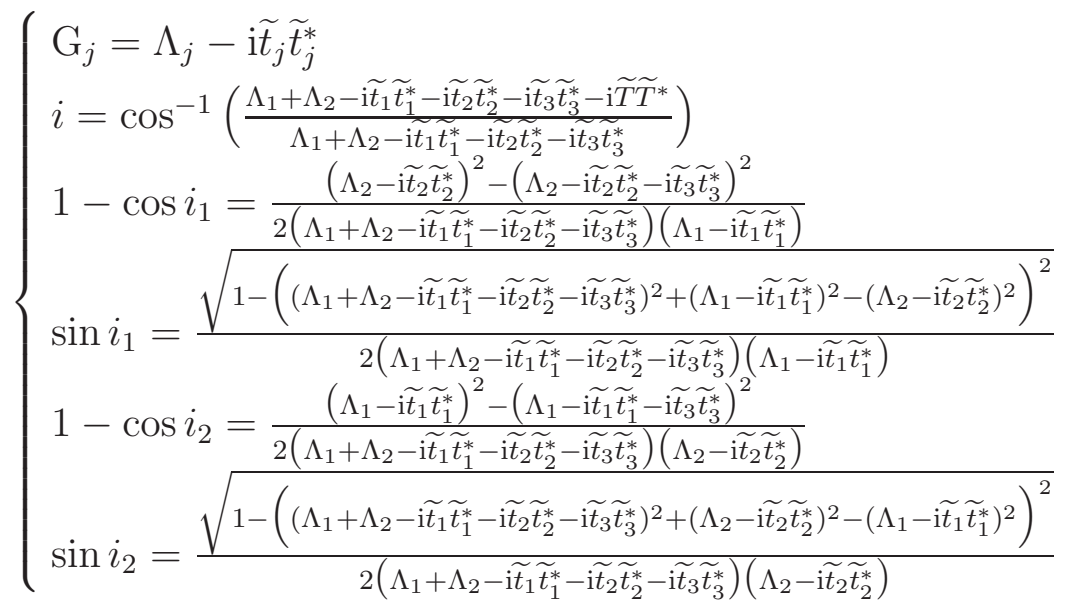




$$
\left\{\begin{array}{l}
\zeta=\arg \frac{\widetilde{T}}{\sqrt{\widetilde{\mathrm{i}}^{\widetilde{T}} \widetilde{T}^{*}}} \\
\gamma+\zeta=\arg \frac{\widetilde{t_{3}}}{\sqrt{\widetilde{\mathrm{i}}_{3} \widetilde{t}_{3}^{*}}} \\
\gamma_{j}+\gamma+\zeta=\arg \frac{\widetilde{t_{j}}}{\sqrt{\widetilde{\mathrm{i}}_{j} \widetilde{t}_{j}^{*}}} \\
\ell_{j}=\lambda_{j}-\arg \frac{\widetilde{t}_{j}}{\sqrt{\widetilde{\mathrm{i}}_{j} \widetilde{t}_{j}^{*}}}
\end{array}\right.
$$

where we have used (A3) to evaluate $\sin i_{2}$ and

$$
1-\cos i_{1}=\frac{\mathrm{G}_{2}^{2}-\left(\mathrm{G}-\mathrm{G}_{1}\right)^{2}}{2 \mathrm{GG}_{1}}, \quad 1-\cos i_{2}=\frac{\mathrm{G}_{1}^{2}-\left(\mathrm{G}-\mathrm{G}_{2}\right)^{2}}{2 \mathrm{GG}_{2}} .
$$

in turn implied by (A3).

\section{c. Analytical expression of $\phi_{\mathrm{rps}_{\pi}^{\mathrm{C}}}$}

To obtain the explicit expression of $\phi_{\mathrm{rps}_{\pi}}$, we adopt a similar procedure as in the previous section. In this case, we rewrite (A5) in the form

$$
\left\{\begin{array}{l}
x_{\mathrm{jrd}}^{(j)}=\mathrm{R}_{0} \mathrm{R}_{j} \widetilde{\mathrm{x}}_{\mathrm{pl}}^{(j)} \\
y_{\mathrm{jrd}}^{(j)}=\mathrm{R}_{0} \mathrm{R}_{j} \widetilde{\mathrm{y}}_{\mathrm{pl}}^{(j)}
\end{array}\right.
$$

where

$$
\begin{aligned}
& \mathrm{R}_{0}=\widetilde{\mathrm{R}}_{0}, \quad \mathrm{R}_{1}=\widetilde{\mathrm{R}}_{1}, \quad \widetilde{\mathrm{x}}_{\mathrm{pl}}^{(1)}=\widetilde{\mathrm{x}}_{\mathrm{pl}}^{(1)}, \quad \widetilde{\mathrm{y}}_{\mathrm{pl}}^{(1)}=\widetilde{\mathrm{y}}_{\mathrm{pl}}^{(1)}, \quad \mathrm{R}_{2}=R_{313}^{-}\left(\zeta+\gamma, \sigma_{2} i_{2}\right) \\
& \widetilde{\mathrm{x}}_{\mathrm{pl}}^{(2)}=R_{3}\left(\zeta+\gamma-\gamma_{2}\right) x_{\mathrm{orb}}^{(2)}\left(-\Lambda_{2}, \mathrm{G}_{2},-\ell_{2}\right), \quad \widetilde{\mathrm{y}}_{\mathrm{pl}}^{(2)}=R_{3}\left(\zeta+\gamma-\gamma_{2}\right) y_{\mathrm{orb}}^{(2)}\left(-\Lambda_{2}, \mathrm{G}_{2},-\ell_{2}\right)
\end{aligned}
$$

with $\widetilde{\mathrm{R}}_{0}, \widetilde{\mathrm{R}}_{1}, \widetilde{\mathrm{x}}_{\mathrm{pl}}^{(1)}, \widetilde{\mathrm{y}}_{\mathrm{pl}}^{(1)}$ as in (A7)-(A9), while, if $\Pi_{2}^{-}:=\left(\begin{array}{ccc}1 & 0 & 0 \\ 0 & -1 & 0 \\ 0 & 0 & 1\end{array}\right)$, then

$$
\begin{aligned}
R_{313}^{-}(\alpha, \beta): & =R_{3}(\alpha) R_{1}(\beta) R_{3}(\alpha) \Pi_{2}^{-} \\
& =\left(\begin{array}{ccc}
1-\sin ^{2} \alpha(1+\cos \beta) & \sin \alpha \cos \alpha(1+\cos \beta) & \sin \alpha \sin \beta \\
\sin \alpha \cos \alpha(1+\cos \beta) & 1-\cos ^{2} \alpha(1+\cos \beta) & -\cos \alpha \sin \beta \\
\sin \alpha \sin \beta & -\cos \alpha \sin \beta & \cos \beta
\end{array}\right) .
\end{aligned}
$$

Note that here we have used

$$
\begin{aligned}
& \Pi_{2}^{-} R_{3}(g) x_{\mathrm{orb}}^{(2)}\left(\Lambda_{2}, \mathrm{G}_{2}, \ell_{2}\right)=R_{3}(-g) x_{\mathrm{orb}}^{(2)}\left(\Lambda_{2}, \mathrm{G}_{2},-\ell_{2}\right)=x_{\mathrm{pl}}^{(2)}\left(\Lambda_{2}, \mathrm{G}_{2},-\ell_{2},-g\right)=x_{\mathrm{pl}}^{(2)}\left(-\Lambda_{2}, \mathrm{G}_{2},-\ell_{2},-g\right) \\
& \Pi_{2}^{-} R_{3}(g) y_{\mathrm{orb}}^{(2)}\left(\Lambda_{2}, \mathrm{G}_{2}, \ell_{2}\right)=-R_{3}(-g) y_{\mathrm{orb}}^{(2)}\left(\Lambda_{2}, \mathrm{G}_{2},-\ell_{2}\right)=y_{\mathrm{pl}}^{(2)}\left(-\Lambda_{2}, \mathrm{G}_{2},-\ell_{2},-g\right)
\end{aligned}
$$


Then we may write, for $\phi_{\mathrm{rps}}^{\mathrm{C}}$, the expression

$$
\left\{\begin{array}{l}
x_{\mathrm{rps}_{\pi}^{\mathbf{C}}}^{(j)}=\mathcal{R}_{0} \mathcal{R}_{j} x_{\mathrm{pl}}^{(j)} \\
y_{\mathrm{rps}_{\pi}^{\mathbf{C}}}^{(j)}=\mathcal{R}_{0} \mathcal{R}_{j} y_{\mathrm{pl}}^{(j)}
\end{array}\right.
$$

where

$$
\mathcal{R}_{0}=\mathrm{R}_{0} \circ \phi_{\mathrm{rps}_{\pi}^{\mathrm{C}}}^{\mathrm{jrd}}, \quad \mathcal{R}_{j}=\mathrm{R}_{j} \circ \phi_{\mathrm{rps}_{\pi}^{\mathrm{C}}}^{\mathrm{jrd}}, \quad x_{\mathrm{pl}}^{(j)}=\widetilde{\mathrm{x}}_{\mathrm{pl}}^{(j)} \circ \phi_{\mathrm{rps}_{\pi}^{\mathrm{C}}}^{\mathrm{jr}}, \quad y_{\mathrm{pl}}^{(j)}=\widetilde{\mathrm{y}}_{\mathrm{pl}}^{(j)} \circ \phi_{\mathrm{rps}}^{\mathrm{jrd}}
$$

The explicit form of $\phi_{\mathrm{rps}_{\pi}^{\mathrm{C}}}$ is obtained replacing the appropriate arguments as (as it follows from $(25))$

$$
\begin{aligned}
& \left\{\begin{array}{l}
\mathrm{G}_{1}=\Lambda_{1}-\mathrm{i} t_{1} t_{1}^{*} \\
\mathrm{G}_{2}=\Lambda_{2}+\mathrm{i} t_{2} t_{2}^{*} \\
i=\cos ^{-1}\left(\frac{\Lambda_{1}-\Lambda_{2}-\mathrm{i} t_{1} t_{1}^{*}-\mathrm{i} t_{2} t_{2}^{*}-\mathrm{i} t_{3} t_{3}^{*}-\mathrm{i} T T^{*}}{\Lambda_{1}-\Lambda_{2}-\mathrm{i} t_{1} t_{1}^{*}-\mathrm{i} t_{2} t_{2}^{*}-\mathrm{i} t_{3} t_{3}^{*}-}\right) \\
1-\cos i_{1}=\frac{\left(\Lambda_{2}-\mathrm{i} t_{2} t_{2}^{*}\right)^{2}-\left(\Lambda_{2}-\mathrm{i} t_{2} t_{2}^{*}-\mathrm{i} t_{3} t_{3}^{*}\right)^{2}}{2\left(\Lambda_{1}-\Lambda_{2}-\mathrm{i} t_{1} t_{1}^{*}-\mathrm{i} t_{2} t_{2}^{*}-\mathrm{i} t_{3} t_{3}^{*}\right)\left(\Lambda_{1}-\mathrm{i} t_{1} t_{1}^{*}\right)} \\
\sin i_{1}=\frac{\sqrt{1-\left(\left(\Lambda_{1}-\Lambda_{2}-\mathrm{i} t_{1} t_{1}^{*}-\mathrm{i} t_{2} t_{2}^{*}-\mathrm{i} t_{3} t_{3}^{*}\right)^{2}+\left(\Lambda_{1}-\mathrm{i} t_{1} t_{1}^{*}\right)^{2}-\left(-\Lambda_{2}-\mathrm{i} t_{2} t_{2}^{*}\right)^{2}\right)^{2}}}{2\left(\Lambda_{1}-\Lambda_{2}-\mathrm{i} t_{1} t_{1}^{*}-\mathrm{i} t_{2} t_{2}^{*}-\mathrm{i} t_{3} t_{3}^{*}\right)\left(\Lambda_{1}-\mathrm{i} t_{1} t_{1}^{*}\right)} \\
1+\cos i_{2}=\frac{\left(\Lambda_{1}-\mathrm{i} t_{1} t_{1}^{*}\right)^{2}-\left(\Lambda_{1}-\mathrm{i} t_{1} t_{1}^{*}-\mathrm{i} t_{3} t_{3}^{*}\right)^{2}}{2\left(\Lambda_{1}-\Lambda_{2}-\mathrm{i} t_{1} t_{1}^{*}-\mathrm{i} t_{2} t_{2}^{*}-\mathrm{i} t_{3} t_{3}^{*}\right)\left(-\Lambda_{2}-\mathrm{i} t_{2} t_{2}^{*}\right)} \\
-\sin i_{2}=\frac{\sqrt{1-\left(\left(\Lambda_{1}-\Lambda_{2}-\mathrm{i} t_{1} t_{1}^{*}-\mathrm{i} t_{2} t_{2}^{*}-\mathrm{i} t_{3} t_{3}^{*}\right)^{2}+\left(-\Lambda_{2}-\mathrm{i} t_{2} t_{2}^{*}\right)^{2}-\left(\Lambda_{1}-\mathrm{i} t_{1} t_{1}^{*}\right)^{2}\right)^{2}}}{2\left(\Lambda_{1}-\Lambda_{2}-\mathrm{i} t_{1} t_{1}^{*}-\mathrm{i} t_{2} t_{2}^{*}-\mathrm{i} t_{3} t_{3}^{*}\right)\left(-\Lambda_{2}-\mathrm{i} t_{2} t_{2}^{*}\right)} \\
\zeta=\arg \frac{T}{\sqrt{\mathrm{i} T T^{*}}} \\
\gamma+\zeta=\arg \frac{t_{3}}{\sqrt{\mathrm{i} t_{3} t_{3}^{*}}} \\
\gamma_{1}+\gamma+\zeta=\arg \frac{t_{1}}{\sqrt{\mathrm{i} t_{1} t_{1}^{*}}} \\
\gamma+\zeta-\gamma_{2}=\arg \frac{t_{2}}{\sqrt{\mathrm{i} t_{2} t_{2}^{*}}} \\
\ell_{1}=\lambda_{1}-\arg \frac{t_{1}}{\sqrt{\mathrm{i} t_{1} t_{1}^{*}}} \\
-\ell_{2}=-\lambda_{2}-\arg \frac{t_{2}}{\sqrt{\mathrm{i} t_{2} t_{2}^{*}}}
\end{array}\right.
\end{aligned}
$$

having used the former equation in (A12) and

$$
1+\cos i_{2}=\frac{\mathrm{G}_{1}^{2}-\left(\mathrm{G}+\mathrm{G}_{2}\right)^{2}}{2 \mathrm{G}\left(-\mathrm{G}_{2}\right)}
$$

again following from (A3). 


\section{d. Proof of Proposition III.1}

Since the third respective components of $\widetilde{\mathrm{x}}_{\mathrm{pl}}^{(j)}, \widetilde{\mathrm{y}}_{\mathrm{pl}}^{(j)}$ in $(\mathrm{A} 10)$ and of $\mathrm{x}_{\mathrm{pl}}^{(j)}, \mathrm{y}_{\mathrm{pl}}^{(j)}$ in (A14) vanish, we may replace the matrices $\widetilde{\mathcal{R}}_{j}$ in (A10) and $\mathcal{R}_{j}$ in (A14) with the matrices that are obtained truncating at 0 their respective third columns. Collecting the formulae obtained after such truncation with (A11) and (A15), the thesis follows.

\section{e. Analytical expression of $\phi_{\mathrm{rps}_{\pi}}$}

Proposition III.1 has, as a consequence, that, formally, the formulae of the real $\operatorname{rps}_{\pi}$ map in (27) can be derived from the ones of the real rps map given in Ref. ${ }^{9}$ (Equations (4.3)-(4.8) and Appendix A) (see also Ref. ${ }^{34}$ (Section 4.3)), for the case $n=2$, letting

$$
\begin{aligned}
& \Lambda_{1}=\Lambda_{1}, \quad \Lambda_{2} \rightarrow-\Lambda_{2}, \quad \lambda_{1}=\lambda_{1}, \quad \lambda_{2}=-\lambda_{2}, \quad \eta_{1}=\eta_{1}, \quad \xi_{1}=\xi_{1} \\
& \eta_{2} \rightarrow \mathrm{i} \eta_{2}, \quad \xi_{2} \rightarrow-\mathrm{i} \xi_{2}, \quad p_{1} \rightarrow \mathrm{i} p, \quad q_{1} \rightarrow-\mathrm{i} q, \quad p_{2} \rightarrow P, \quad q_{2} \rightarrow Q
\end{aligned}
$$

The result is as follows:

$$
\left\{\begin{array}{l}
x_{\mathrm{rps}_{\pi}}^{(j)}=\mathcal{R}_{0} \mathcal{R}_{j} x_{\mathrm{pl}}^{(j)} \\
y_{\mathrm{rps}_{\pi}}^{(j)}=\mathcal{R}_{0} \mathcal{R}_{j} y_{\mathrm{pl}}^{(j)}
\end{array} \quad j=1,2\right.
$$

where $\mathcal{R}_{0}, \mathcal{R}_{j}, x_{\mathrm{pl}}^{(j)}$ and $y_{\mathrm{pl}}^{(j)}$ are defined as follows. Let $s_{1}=-s_{2}=1$;

$$
\begin{aligned}
\mathrm{c}_{2}^{*} & :=\frac{1}{2\left(\Lambda_{1}-\Lambda_{2}\right)-\sum_{i=1}^{2} s_{i}\left(\eta_{i}^{2}+\xi_{i}^{2}\right)+p^{2}+q^{2}} \\
\mathrm{c}_{1}^{*} & :=\frac{2 \Lambda_{2}-\left(\eta_{2}^{2}+\xi_{2}^{2}\right)-\frac{\left(p^{2}+q^{2}\right)}{2}}{\left(2\left(\Lambda_{1}-\Lambda_{2}\right)-\sum_{i=1}^{2} s_{i}\left(\eta_{i}^{2}+\xi_{i}^{2}\right)+p^{2}+q^{2}\right)\left(2 \Lambda_{1}-\left(\eta_{1}^{2}+\xi_{1}^{2}\right)\right)} \\
\mathrm{c}_{2} & :=\frac{2 \Lambda_{1}-\left(\eta_{1}^{2}+\xi_{1}^{2}\right)+\frac{\left(p^{2}+q^{2}\right)}{2}}{\left(2\left(\Lambda_{1}-\Lambda_{2}\right)-\sum_{i=1}^{2} s_{i}\left(\eta_{i}^{2}+\xi_{i}^{2}\right)+p^{2}+q^{2}\right)\left(2 \Lambda_{2}-\left(\eta_{2}^{2}+\xi_{2}^{2}\right)\right)} \\
\mathrm{s}_{i}^{*} & :=(-1)^{i} \sqrt{\mathrm{c}_{i}^{*}\left(2-\left(p_{i}^{2}+q_{i}^{2}\right) \mathrm{c}_{i}^{*}\right)}, \quad \mathrm{s}_{2}:=\sqrt{\mathrm{c}_{2}\left(2-\left(p^{2}+q^{2}\right) \mathrm{c}_{2}\right)}
\end{aligned}
$$

Then

$$
\mathcal{R}_{0}:=\left(\begin{array}{ccc}
1-Q^{2} \mathrm{c}_{2}^{*} & -P Q \mathrm{c}_{2}^{*} & -Q \mathrm{~s}_{2}^{*} \\
-P Q \mathrm{c}_{2}^{*} & 1-P^{2} \mathrm{c}_{2}^{*} & -P \mathrm{~s}_{2}^{*} \\
Q \mathrm{~s}_{2}^{*} & P \mathrm{~s}_{2}^{*} & 1-\left(P^{2}+Q^{2}\right) \mathrm{c}_{2}^{*}
\end{array}\right), \mathcal{R}_{1}:=\left(\begin{array}{ccc}
1-q^{2} \mathrm{c}_{1}^{*} & p q \mathrm{c}_{1}^{*} & -q \mathrm{~s}_{1}^{*} \\
p q \mathrm{c}_{1}^{*} & 1-p^{2} \mathrm{c}_{1}^{*} & p \mathrm{~s}_{1}^{*} \\
q \mathrm{~s}_{1}^{*} & -p \mathrm{~s}_{1}^{*} & 1-\left(p^{2}+q^{2}\right) \mathrm{c}_{1}^{*}
\end{array}\right)
$$


$\mathcal{R}_{2}:=\left(\begin{array}{ccc}1-q^{2} \mathrm{c}_{2} & p q \mathrm{c}_{2} & -q \mathrm{~s}_{2} \\ p q \mathrm{c}_{2} & 1-p^{2} \mathrm{c}_{2} & p \mathrm{~s}_{2} \\ q \mathrm{~s}_{2} & -p \mathrm{~s}_{2} & 1-\left(p^{2}+q^{2}\right) \mathrm{c}_{2}\end{array}\right)$

while

$$
\left(\Lambda_{j}, \lambda_{j}, \eta_{j}, \xi_{j}\right) \rightarrow\left(y_{\mathrm{pl}}^{(j)}\left(\Lambda_{j}, \lambda_{j}, \eta_{j}, \xi_{j}\right), x_{\mathrm{pl}}^{(j)}\left(\Lambda_{j}, \lambda_{j}, \eta_{j}, \xi_{j}\right)\right) \quad j=1,2
$$

is the "2-reversed planar Poincaré map", given by

$$
\left(y_{\mathrm{pl}}^{(j)}\left(\Lambda_{j}, \lambda_{j}, \eta_{j}, \xi_{j}\right), x_{\mathrm{pl}}^{(j)}\left(\Lambda_{j}, \lambda_{j}, \eta_{j}, \xi_{j}\right)\right)= \begin{cases}\left(\widetilde{y}_{\mathrm{pl}}^{(1)}\left(\Lambda_{1}, \lambda_{1}, \eta_{1}, \xi_{1}\right), \widetilde{x}_{\mathrm{pl}}^{(2)}\left(\Lambda_{1}, \lambda_{1}, \eta_{1}, \xi_{1}\right)\right) & j=1 \\ \left(\Pi_{2}^{-} \widetilde{y}_{\mathrm{pl}}^{(2)}\left(\Lambda_{2}, \lambda_{2}, \eta_{2}, \xi_{2}\right), \Pi_{2}^{-} \widetilde{x}_{\mathrm{pl}}^{(2)}\left(\Lambda_{2}, \lambda_{2}, \eta_{2}, \xi_{2}\right)\right) j=2\end{cases}
$$

with $\Pi_{2}^{-}=\left(\begin{array}{cc}1 & 0 \\ 0 & -1\end{array}\right)$; having used the relations

$$
\begin{aligned}
& \widetilde{x}_{\mathrm{pl}}^{(2)}\left(-\Lambda_{2},-\lambda_{2}, \mathrm{i} \eta_{2},-\mathrm{i} \xi_{2}\right)=\Pi_{2}^{-} \widetilde{x}_{\mathrm{pl}}^{(2)}\left(\Lambda_{2}, \lambda_{2}, \eta_{2}, \xi_{2}\right) \\
& \widetilde{y}_{\mathrm{pl}}^{(2)}\left(-\Lambda_{2},-\lambda_{2}, \mathrm{i} \eta_{2},-\mathrm{i} \xi_{2}\right)=\Pi_{2}^{-} \widetilde{y}_{\mathrm{pl}}^{(2)}\left(\Lambda_{2}, \lambda_{2}, \eta_{2}, \xi_{2}\right) .
\end{aligned}
$$

\section{f. D'Alembert rules in the retrograde problem}

We let

$$
\mathrm{H}_{\mathrm{rps}_{\pi}^{\mathbf{C}}}\left(\Lambda, \lambda, t, t^{*}\right):=\mathrm{H} \circ \phi_{\operatorname{rps}_{\pi}^{\mathbf{C}}}\left(\Lambda, \lambda, t, t^{*}\right)=\mathrm{h}_{\mathrm{k}}\left(\Lambda_{1}, \Lambda_{2}\right)+\mu f_{\mathrm{rps}_{\pi}^{\mathbf{C}}}\left(\Lambda, \lambda, t, t^{*}\right)
$$

the Hamiltonian (2) expressed in the complex $\operatorname{rps}_{\pi}$ coordinates. Proposition III.1 implies that the the symmetries that have beed discussed in Ref. ${ }^{9}$ (Section 6) or Ref. ${ }^{8}$ (Eqs. (3.19)(3.24)) have the following counterpart in the case of the retrograde problem.

a. Invariance by rotations around $\mathrm{C}$ The following group of symmetries holds:

$$
f_{\mathrm{rps}_{\pi}^{\mathbf{C}}}^{\mathbf{C}}\left(\Lambda, \lambda, t, t^{*}\right)=f_{\mathrm{rps}_{\pi}^{\mathbf{C}}}\left(\Lambda, \lambda_{1}+g, \lambda_{2}-g, \mathcal{S}_{g}\left(t, t^{*}\right)\right), \quad \forall g \in \mathbf{T},
$$

with $\mathcal{S}_{g}\left(t_{1}, t_{2}, t_{3}, t_{1}^{*}, t_{2}^{*}, t_{3}^{*}\right)=\left(t_{1} e^{\mathrm{i} g}, t_{2} e^{\mathrm{i} g}, t_{3} e^{\mathrm{i} g}, t_{1}^{*} e^{-\mathrm{i} g}, t_{2}^{*} e^{-\mathrm{i} g}, t_{3}^{*} e^{-\mathrm{i} g}\right)$. It is implied by the invariance of $f_{\mathrm{rps}_{\pi}^{\mathrm{C}}}$ under rotations around $\mathrm{C}$ (corresponding to the Hamiltonian flow of $\mathrm{G}$ in (49), which, in complex coordinates, is $\mathrm{G}=\Lambda_{1}-\Lambda_{2}-\mathrm{i} t_{1} t_{1}^{*}-\mathrm{i} t_{2} t_{2}^{*}-\mathrm{i} t_{3} t_{3}^{*}$ ), which, in terms of $\operatorname{rps}_{\pi}^{\mathbf{C}}$, are

$$
\mathcal{R}_{g}: \quad\left(\Lambda, \lambda, t, t^{*}\right) \rightarrow\left(\Lambda, \lambda_{1}+g, \lambda_{2}-g, \mathcal{S}_{g}\left(t, t^{*}\right), T e^{\mathrm{i} g}, T^{*} e^{-\mathrm{i} g}\right)
$$


b. Invariance by reflections The following identities hold

$$
\begin{aligned}
f_{\mathrm{rps}_{\pi}^{\mathbf{C}}}\left(\Lambda, \lambda, t, t^{*}\right) & =f_{\mathrm{rps}_{\pi}}\left(\Lambda, \frac{\pi}{2}-\lambda_{1},-\frac{\pi}{2}-\lambda_{2}, \mathcal{S}_{1 \leftrightarrow 2}\left(t, t^{*}\right)\right) \\
f_{\mathrm{rps}_{\pi}^{\mathbf{C}}}\left(\Lambda, \lambda, t, t^{*}\right) & =f_{\mathrm{rps}_{\pi}}\left(\Lambda, \lambda, \mathcal{S}_{3}^{-}\left(t, t^{*}\right)\right) .
\end{aligned}
$$

with

$$
\left\{\begin{array}{l}
\mathcal{S}_{1 \leftrightarrow 2}\left(t_{1}, t_{2}, t_{3}, t_{1}^{*}, t_{2}^{*}, t_{3}^{*}\right):=\left(-t_{1}^{*},-t_{2}^{*}, t_{3},-t_{1},-t_{2}, t_{3}^{*}\right) \\
\mathcal{S}_{3}^{-}\left(t_{1}, t_{2}, t_{3}, t_{1}^{*}, t_{2}^{*}, t_{3}^{*}\right):=\left(t_{1}, t_{2},-t_{3}, t_{1}^{*}, t_{2}^{*},-t_{3}^{*}\right) .
\end{array}\right.
$$

Such indentities are implied by the invariance of $f_{\mathrm{rps}_{\pi}}$ under the tranformations

$$
\begin{array}{ll}
\mathcal{R}_{3}^{-}: & \left(\Lambda, \lambda, t, t^{*}\right) \rightarrow\left(\Lambda, \lambda, \mathcal{S}_{3}^{-}\left(t, t^{*}\right),-T,-T^{*}\right) \\
\mathcal{R}_{1 \leftrightarrow 2}: & \left(\Lambda, \lambda, t, t^{*}\right) \rightarrow\left(\Lambda, \frac{\pi}{2}-\lambda_{1},-\frac{\pi}{2}-\lambda_{2}, \mathcal{S}_{1 \leftrightarrow 2}\left(t, t^{*}\right), T, T^{*}\right)
\end{array}
$$

in turn corresponding, in Cartesian coordinates, respectively, to

$$
\begin{array}{lll}
\mathcal{R}_{3}^{-}: & x^{(i)} \rightarrow\left(x_{1}^{(i)}, x_{2}^{(i)},-x_{3}^{(i)}\right), & y^{(i)} \rightarrow\left(y_{1}^{(i)}, y_{2}^{(i)},-y_{3}^{(i)}\right) \\
\mathcal{R}_{1 \leftrightarrow 2}: & x^{(i)} \rightarrow\left(x_{2}^{(i)}, x_{1}^{(i)}, x_{3}^{(i)}\right), & y^{(i)} \rightarrow\left(-y_{2}^{(i)},-y_{1}^{(i)},-y_{3}^{(i)}\right) .
\end{array}
$$

\section{ACKNOWLEDGMENTS}

I wish to thank the managing editor and, especially, the anonymous referees, whose careful remarks helped me to improve in a substantial way the presentation of the results. Thanks also to A. Celletti, A. Giorgilli and V. Kaloshin for their interest.

This research was supported by H2020 Excellent Science (Grant 677793 StableChaoticPlanetM).

\section{REFERENCES}

${ }^{1}$ V. I. Arnold. A theorem of Liouville concerning integrable problems of dynamics. Sibirsk. Mat. Ž., 4:471-474, 1963.

${ }^{2}$ V. I. Arnold. Proof of a theorem by A. N. Kolmogorov on the invariance of quasi-periodic motions under small perturbations of the Hamiltonian. Russian Math. Survey, 18:13-40, 1963.

${ }^{3}$ V.I. Arnold. Small denominators and problems of stability of motion in classical and celestial mechanics. Russian Math. Surveys, 18(6): 85-191, 1963. 
${ }^{4}$ V. I. Arnold. Instability of dynamical systems with many degrees of freedom. Dokl. Akad. Nauk SSSR, 156:9-12, 1964.

${ }^{5} \mathrm{~F}$. Boigey. Élimination des nœuds dans le problème newtonien des quatre corps. Celestial Mech., 27(4):399-414, 1982.

${ }^{6}$ L. Chierchia and G. Gallavotti. Drift and diffusion in phase space. Ann. Inst. H. Poincaré Phys. Théor., 60(1): 144, 1994.

${ }^{7}$ L. Chierchia and G. Pinzari. Properly-degenerate KAM theory (following V.I. Arnold). Discrete Contin. Dyn. Syst. Ser. S, 3(4): 545-578, 2010.

${ }^{8}$ L. Chierchia and G. Pinzari. Planetary Birkhoff normal forms. J. Mod. Dyn., 5(4): 623$664,2011$.

${ }^{9} \mathrm{~L}$. Chierchia and G. Pinzari. The planetary $N$-body problem: symplectic foliation, reductions and invariant tori. Invent. Math., 186(1): 1-77, 2011.

${ }^{10}$ L. Chierchia and G. Pinzari. Metric stability of the planetary n-body problem. Proceedings of the International Congress of Mathematicians, 2014.

${ }^{11}$ A. Deprit. Elimination of the nodes in problems of $n$ bodies. Celestial Mech., 30(2): 181-195, 1983.

${ }^{12}$ J. Féjoz. Démonstration du 'théorème d'Arnold' sur la stabilité du système planétaire (d'après Herman). Ergodic Theory Dynam. Systems, 24(5): 1521-1582, 2004.

${ }^{13}$ J. Féjoz. On "Arnold's theorem" in celestial mechanics -a summary with an appendix on the poincaré coordinates. Discrete and Continuous Dynamical Systems, 33: 3555-3565, 2013.

${ }^{14} \mathrm{~S}$. Ferrer and C. Osácar. Harrington's Hamiltonian in the stellar problem of three bodies: reductions, relative equilibria and bifurcations. Celestial Mech. Dynam. Astronom., 58(3): 245-275, 1994.

${ }^{15} \mathrm{G}$. Gallavotti. The elements of mechanics. Texts and Monographs in Physics. 1983

${ }^{16}$ Robert S. Harrington. The stellar three-body problem. Celestial Mech. and Dyn. Astrronom, 1(2):200-209, 1969.

${ }^{17}$ M. R. Herman. Torsion du problème planétaire, edited by J. Féjoz in 2009. Available in the electronic 'Archives Michel Herman' at http://www.college-de-france.fr/default/EN/all/equ_dif/archives_michel_herman.htm.

${ }^{18}$ H. Hofer, E. Zehnder. Symplectic Invariants and Hamiltonian Dynamics. Birkhäuser Verlag, Basel, 1994. 
${ }^{19}$ C. G. J. Jacobi. Sur l'élimination des noeuds dans le problème des trois corps. Astronomische Nachrichten, Bd XX: 81-102, 1842.

${ }^{20}$ W. H. Jefferys and J. Moser. Quasi-periodic solutions for the three-body problem. Astronom. J., 71: 568-578, 1966.

${ }^{21}$ A.N. Kolmogorov. On the Conservation of Conditionally Periodic Motions under Small Perturbation of the Hamiltonian. Dokl. Akad. Nauk SSR, 98: 527-530, 1954.

${ }^{22}$ J. Laskar and P. Robutel. Stability of the planetary three-body problem. I. Expansion of the planetary Hamiltonian. Celestial Mech. Dynam. Astronom., 62(3): 193-217, 1995.

${ }^{23}$ T. Levi-Civita. Sopra la equazione di Kepler. Astronomische Nachrichten, 165(20):313-314, 1904 .

${ }^{24}$ M. L. Lidov and S. L. Ziglin. Non-restricted double-averaged three body problem in Hill's case. Celestial Mech., 13(4):471-489, 1976.

${ }^{25}$ F. Malige, P. Robutel, and J. Laskar. Partial reduction in the $n$-body planetary problem using the angular momentum integral. Celestial Mech. Dynam. Astronom., 84(3): 283-316, 2002.

${ }^{26}$ J.N. Mather. Existence of quasiperiodic orbits for twist homeomorphisms of the annulus. Topology, 21: 457-467, 1982

${ }^{27}$ A. S. Miščenko and A. T. Fomenko. Integration of Hamiltonian systems with noncommutative symmetries. Trudy Sem. Vektor. Tenzor. Anal., (20): 5-54, 1981.

${ }^{28} \mathrm{~J}$. Moser. The analytic invariants of an area-preserving mapping near a hyperbolic fixed point. Comm. Pure Appl. Math., 9: 673-692, 1956.

${ }^{29} \mathrm{~J}$. Moser. On invariant curves of area-preserving mappings of an annulus. Nachr. Akad. Wiss. Göttingen Math.-Phys. Kl. II, 1962:1-20, 1962.

${ }^{30}$ N. N. Nehorošev. Action-angle variables, and their generalizations. Trudy Moskov. Mat. Ob̌̌č., 26: 181-198, 1972.

${ }^{31}$ J. F. Palacián, F. Sayas, and P. Yanguas. Regular and singular reductions in the spatial three-body problem. Qualitative Theory of Dynamical Systems, 12(1): 143-182, 2013.

${ }^{32}$ J. F. Palacián, F. Sayas, and P. Yanguas. Flow reconstruction and invariant tori in the spatial three-body problem. J. Differential Equations, 258(6): 2114-2159, 2015.

${ }^{33}$ G. Pinzari. Perihelia reduction and global Kolmogorov tori in the planetary problem. Memoirs American Mathematical Society. in press. 
${ }^{34} \mathrm{G}$. Pinzari. On the Kolmogorov set for many-body problems. PhD thesis, Università Roma Tre, April 2009.

${ }^{35}$ G. Pinzari. Aspects of the planetary Birkhoff normal form. Regul. Chaotic Dyn., 18(6): 860-906, 2013.

${ }^{36} \mathrm{H}$. Poincaré. Les méthodes nouvelles de la mécanique céleste, 1899. Gauthier-Villars, Paris.

In the referenced text, Poincaré draws the following imaginary picture of the phase portrait of a non-integrable system closely to a homoclinic point: "Que l'on cherche à se représenter la figure formée par ces courbes et leurs intersections en nombre infini dont chacune correspond à une solution doublement asymptotique, ces intersections forment une sorte de treillis, de tissu, de réseau à mailles infiniment serrées; chacune des deux courbes ne doit jamais se recouper elle-mme, mais elle doit se replier sur elle-mme d'une manire trs complexe pour venir recouper une infinité de fois toutes les mailles du réseau. On sera frappé de la complexité de cette figure, que je ne cherche mme pas à tracer. Rien n'est plus propre à nous donner une idée de la complication du problme des trois corps et en général de tous les problmes de Dynamique o il n'y a pas d'intégrale uniforme et o les séries de Bohlin sont divergentes."

${ }^{37}$ J. Pöschel. Nekhoroshev estimates for quasi-convex Hamiltonian systems. Math. Z., 213(2): 187-216, 1993.

${ }^{38}$ R. Radau. Sur une transformation des équations différentielles de la dynamique. Ann. Sci. Ec. Norm. Sup., 5: 311-375, 1868.

${ }^{39}$ P. Robutel. Stability of the planetary three-body problem. II. KAM theory and existence of quasiperiodic motions. Celestial Mech. Dynam. Astronom., 62(3): 219-261, 1995.

${ }^{40}$ E. Valdinoci. Families of whiskered tori for a-priori stable/unstable Hamiltonian systems and construction of unstable orbits. Math. Phys. Electron. J., 6: Paper 2, 31 pp. (electronic), 2000.

${ }^{41}$ Aubry-Mather theory establishes the existence of quasi-periodic motions filling completely invariant sets with any prescribed frequency. The pioneering work of Mather ${ }^{26}$ deals with area-preserving twist homeomorphism $\mathrm{f}$ of an annulus. Attempts (still under investigation) of extending Mather's result to more dimensions deal with Hamiltonian systems

$$
\left\{\begin{array}{l}
\dot{x}=\partial_{p} \mathrm{H}(p, x) \\
\dot{p}=-\partial_{x} \mathrm{H}(p, x)
\end{array}\right.
$$


where $\mathrm{H}(p, x): \quad \mathbf{R}^{n} \times \mathcal{M} \rightarrow \mathbf{R}$ is a $C^{2-}$ smooth Hamiltonian, $\mathcal{M} \subset \mathbf{R}^{n}$ is a $n^{-}$ dimensional compact surface, the Hessian $\partial_{p, p}^{2} \mathrm{H}$ is convex, $\mathrm{H}$ is super-linear in $p$, i.e., $\lim _{|p| \rightarrow \infty} \mathrm{H}(p, x) /|p|=+\infty$, and the flow is complete, i.e., for any $\left(p_{0}, x_{0}\right) \in \mathcal{M} \subset \mathbf{R}^{n}$, the Hamiltonian flow $(p(t), x(t))$ with initial datum $\left(p_{0}, x_{0}\right)$ exists at any time $t \geq 0$.

${ }^{42}$ By Liouville-Arnold Theorem, Ref. ${ }^{1}$, the phase space of a system verifying its assumptions foliates (modulo a diffeomorphism) as the union of $n$-dimensional sub-manifolds $\left\{\mathrm{I}_{0}\right\} \times \mathbf{T}^{n}$, invariant for the h-flow, where the motion has linear law:

$$
\mathrm{I}=\mathrm{I}_{0}, \quad \varphi=\varphi_{0}+\omega_{0}\left(\mathrm{I}_{0}\right)\left(t-t_{0}\right), \quad \mathrm{I}_{0} \in \mathcal{I}, \quad \varphi_{0} \in \mathbf{T}^{n}
$$

with $\omega_{0}(\mathrm{I}):=\partial_{\mathrm{I}} \mathrm{h}(\mathrm{I})$. The continuation, under suitable assumptions, of many of such motions to non-integrable systems which however have the "close to integrable" form

$$
\mathrm{H}(I, \varphi)=\mathrm{h}(\mathrm{I})+\mu f(\mathrm{I}, \varphi) \quad(0<\mu \ll 1)
$$

is the precisely the scope of KAM theory. More precisely, KAM theory ensures the possibility of continuing to the full systems at least those motions where $\partial_{\mathrm{I}}^{2} \mathrm{~h}$ does not vanish identically (the so-called Kolmogorov condition, or possible weakened versions of it) and the frequencies $\omega$ satisfy the Diophantine inequality:

$$
|\omega \cdot k| \geq \frac{\gamma}{|k|^{\tau}} \quad \forall k \in \mathbf{Z}^{n} \backslash\{0\} \quad \text { for suitable } \quad \gamma, \tau>0
$$

KAM theory originated with Kolmogorov's pioneering 1954 paper Ref. ${ }^{21}$. The literature concerning such a theory is so wide that we give up any attempt at completeness.

${ }^{43} \mathrm{~A}$ motion $t \rightarrow q(t)=\left(x_{1}(t), x_{2}(t), \cdots\right)$ of a system of $N$ particles in $\mathbf{R}^{\nu}$ is quasi-periodic with $m \geq 2$ frequencies if, for all $t \geq 0, q(t)$ has the form

$$
q(t)=\widehat{q}\left(\omega_{1} t, \ldots, \omega_{m} t\right)
$$

for some $2 \pi$-periodic function of $m$ arguments and suitable $\omega:=\left(\omega_{1}, \ldots, \omega_{n}\right) \in \mathbf{R}^{m} \backslash \mathbf{Q}^{m}$. The case $m=1$ or $m \geq 2$ and $\omega \in \mathbf{Q}^{m}$ corresponds to a periodic motion. In the former case of $\omega_{i}$ 's not rationally related, the motion evolves on the $m$-dimensional manifold $\mathcal{T}:=\bigcup_{t \geq 0}\{q(t)\}$ called quasi-periodic torus.

A quasi-periodic torus $\mathcal{T}$ is said

- maximal if it is not possible to find quasi-periodic tori with $m^{\prime}>m$ frequencies;

- whiskered if it is not maximal and, moreover, there exist two non-empty manifolds $\mathcal{W}^{+}=$ $\bigcup_{t \geq 0}\{q(t)\}, \mathcal{W}^{-}=\bigcup_{t \leq 0}\{q(t)\}$ made of motions of the system such that $\mathcal{T}=\mathcal{W}^{+} \cap \mathcal{W}^{-}$ 
and

$$
\lim _{t \rightarrow \pm \infty} \operatorname{dist}(q(t), \mathcal{T})=0 \quad \forall q \in \mathcal{W}^{ \pm} .
$$

Note that our definition of maximal torus is a bit different from the standard one (according to which, a torus is maximal when the number of frequencies equals the number of degrees of freedom). This is because for systems which (like the $N$-body problem, and hence the case considered in the paper) possess non-commuting first integrals, the maximum number of not identically vanishing frequencies may be less than the number of degrees of freedom. ${ }^{44} \mathrm{~A}$ celestial body is said to have a retrograde (as opposite to direct, or prograde) motion if its angular momentum $\mathrm{C}(t)=x(t) \times \mathrm{m} \dot{x}(t)$ has a negative projection along the direction of the (constant) total angular momentum $\mathrm{C}_{\text {tot }}=\sum_{\mathrm{m}} \mathrm{C}(t)$ of the smallest closed system the celestial body is part of. The retrograde motion is frequently observed in the dynamics of gravitational systems. In our solar system, many natural satellites of Jupiter and Saturn (e.g., Carme, Anake, Pasiphae groups for the former; Phoebe, Skathi, Skoll, and many others, for the latter) have such kind of motion.

${ }^{45} \mathrm{~A}$ family $\left\{\mathrm{F}_{i}\right\}_{i=1, \ldots, n}$ is called independent if the gradients $\left\{\partial \mathrm{F}_{i}\right\}_{i=1, \ldots, n}$ are linearly independent at each point of phase space. Moreover, $\mathrm{F}$ and $\mathrm{F}^{\prime}$ are called Poisson-commuting if the Poisson parentheses $\left\{\mathrm{F}, \mathrm{F}^{\prime}\right\}=\sum_{i=1}^{n}\left(\partial_{y_{i}} \mathrm{~F} \partial_{x_{i}} \mathrm{~F}^{\prime}-\partial_{x_{i}} \mathrm{~F} \partial_{y_{i}} \mathrm{~F}^{\prime}\right)$ vanish.

${ }^{46}$ The only work known to the author where Deprit's coordinates are mentioned, up to Ref. ${ }^{34}$, is Ref. ${ }^{14}$. However, such case is not really exhaustive, because it deals with the three-body problem, in which case Deprit's coordinates are the same as the classical Jacobi reduction of the nodes, Ref. ${ }^{19}$.

${ }^{47}$ In this respect, Deprit declared: "Whether the new phase variables [...] are practical in the General Theory of Perturbation is an open question. At least, for planetary theories, the answer is likely to be in the negative [..." , Ref. ${ }^{11}$ (p. 194). 